\title{
OPTIMAL WATERFLOOD MANAGEMENT UNDER GEOLOGIC UNCERTAINTY USING RATE CONTROL: THEORY AND FIELD APPLICATIONS
}

\author{
A Dissertation \\ by \\ AHMED HUMAID H. ALHUTHALI \\ Submitted to the Office of Graduate Studies of \\ Texas A\&M University \\ in partial fulfillment of the requirements for the degree of \\ DOCTOR OF PHILOSOPHY
}

May 2009

Major Subject: Petroleum Engineering 


\title{
OPTIMAL WATERFLOOD MANAGEMENT UNDER GEOLOGIC UNCERTAINTY USING RATE CONTROL: THEORY AND FIELD APPLICATIONS
}

\author{
A Dissertation \\ by \\ AHMED HUMAID H. ALHUTHALI \\ Submitted to the Office of Graduate Studies of \\ Texas A\&M University \\ in partial fulfillment of the requirements for the degree of \\ DOCTOR OF PHILOSOPHY
}

Approved by:

Chair of Committee, Akhil Datta-Gupta

Committee Members, Alfred Hill

Daulat D. Mamora

Wolfgang Bangerth

Head of Department, Stephen A. Holditch

May 2009

Major Subject: Petroleum Engineering 


\begin{abstract}
Optimal Waterflood Management under Geologic Uncertainty Using Rate Control:

Theory and Field Applications. (May 2009)

Ahmed Humaid H. Alhuthali, B.S., King Fahd University of Petroleum and Minerals; M.S., Texas A\&M University Chair of Advisory Committee: Dr. Akhil Datta-Gupta
\end{abstract}

Waterflood optimization via rate control is receiving increased interest because of rapid developments in the smart well completions and I-field technology. The use of inflow control valves (ICV) allows us to optimize the production/injection rates of various segments along the wellbore, thereby maximizing sweep efficiency and delaying water breakthrough. It is well recognized that field scale rate optimization problems are difficult because they often involve highly complex reservoir models, production and facilities related constraints and a large number of unknowns. Some aspects of the optimization problem have been studied before using mainly optimal control theory. However, the applications to-date have been limited to rather small problems because of the computation time and the complexities associated with the formulation and solution of adjoint equations. Field-scale rate optimization for maximizing waterflood sweep efficiency under realistic field conditions has still remained largely unexplored.

We propose a practical and efficient approach for computing optimal injection and production rates and thereby manage the waterflood front to maximize sweep efficiency and delay the arrival time to minimize water cycling. Our work relies on equalizing the arrival times of the waterfront at all producers within selected sub-regions of a water flood project. The arrival time optimization has favorable quasi-linear properties and the optimization proceeds smoothly even if our initial conditions are far from the solution. We account for geologic uncertainty using two optimization schemes. The first one is to formulate the objective function in a stochastic form which relies on a combination of expected value and standard deviation combined with a risk attitude 
coefficient. The second one is to minimize the worst case scenario using a min-max problem formulation. The optimization is performed under operational and facility constraints using a sequential quadratic programming approach. A major advantage of our approach is the analytical computation of the gradient and Hessian of the objective which makes it computationally efficient and suitable for large field cases.

Multiple examples are presented to support the robustness and efficiency of the proposed optimization scheme. These include several 2D synthetic examples for validation purposes and 3D field applications. 


\section{DEDICATION}

To my beloved wife, Lama Alakkas, for her unconditional love and relentless support.

To my adorable children, Juri, Humaid, and Ramah, for the joy and happiness they brought to my life. 


\section{ACKNOWLEDGEMENTS}

All glory, praise and honor to Allah almighty for his infinite mercies and abundant blessings.

I would like to express my gratitude to the management of Saudi Aramco for the scholarship award that has afforded me the opportunity of pursuing my graduate studies.

I would like to thank my academic advisor, Dr. Akhil Datta-Gupta for his encouraging words and unparalleled academic guidance through my years of study.

I would also like to thank the members of my committee, Dr. Daniel Hill, Dr. Daulat D. Mamora, and Dr. Wolfgang Bangerth for the valuable comments and the insightful questions that have shaped the work in this dissertation.

Special thanks to my colleagues in the research group, for the constructive discussions and their friendship. 


\section{TABLE OF CONTENTS}

Page

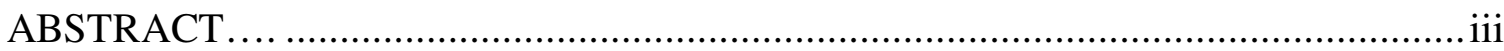

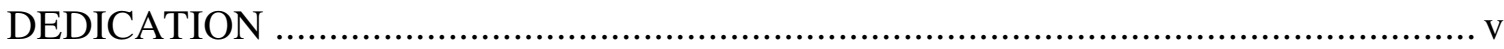

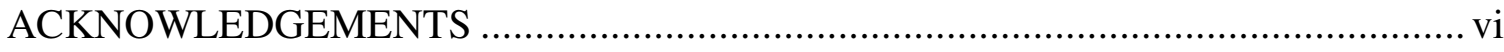

TABLE OF CONTENTS .............................................................................. vii

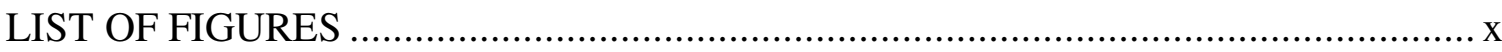

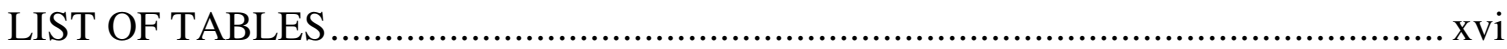

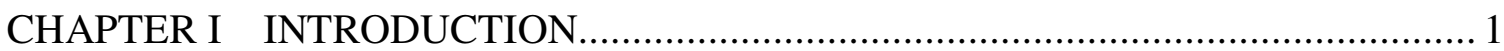

1.1 Optimal Waterflood Management Using Rate Controls ................................ 3

1.2 Optimal Waterflood Management under Geologic Uncertainty...................... 5

1.3 Objectives ........................................................................... 6

1.3.1 Optimal Waterflood Management for a Single Reservoir Model ................. 6

1.3.2 Optimal Waterflood Management under Geological Uncertainty ................. 7

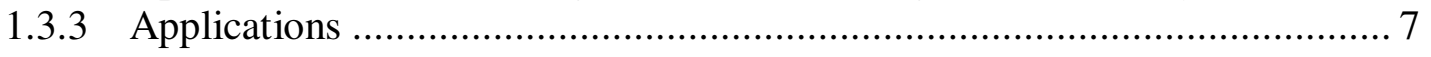

CHAPTER II OPTIMAL WATERFLOOD MANAGEMENT VIA RATE CONTROL USING SINGLE RESERVOIR MODEL......................... 8

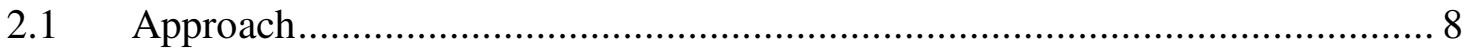

2.2 An Illustration of the Procedure ............................................................. 10

2.3 Mathematical Formulation.................................................................. 21

2.3.1 Objective Function and Its Minimization ...................................... 21

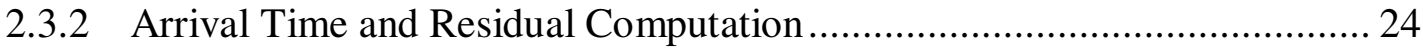

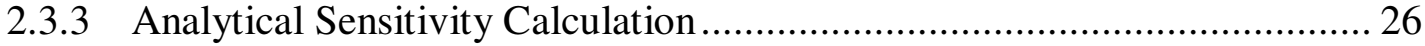

$2.4 \quad$ Application and Discussion ..................................................... 28

2.4.1 Sensitivity Comparisons and Convergence Behavior: 2D Homogeneous

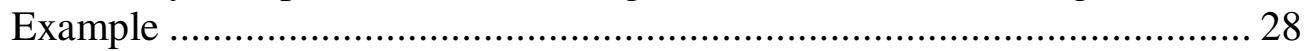

2.4.2 Impact of Mobility Ratio: 2D Heterogeneous Example ............................ 33

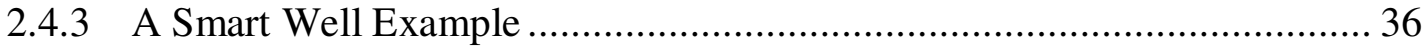

2.4.4 A Field Example ........................................................................ 38

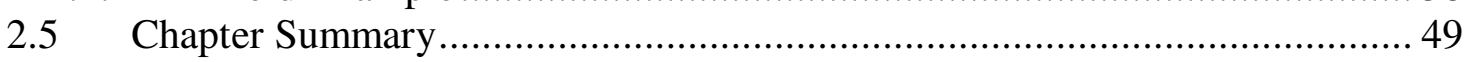


CHAPTER III OPTIMAL RATE UNDER GEOLOGIC UNCERTAINTY ................. 51

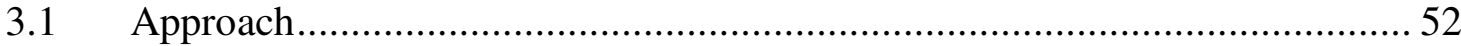

3.2 Mathematical Formulation.................................................................. 53

3.2.1 Objective Function Formulation......................................................... 54

3.2.2 Objective Function Minimization.................................................. 56

3.2.3 Objective Function Evaluation ............................................................. 58

3.2.4 Objective Function Gradients and Hessian......................................... 58

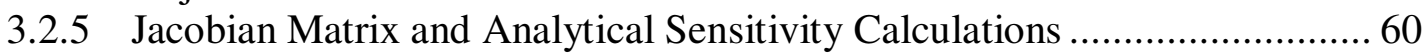

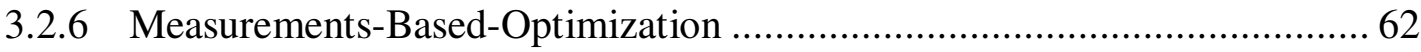

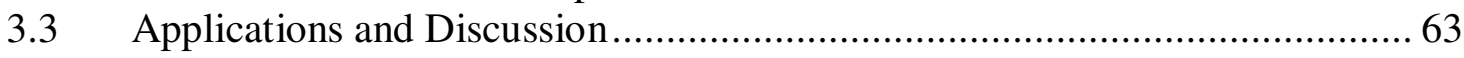

3.3.1 Illustration of the Approach: A 2D Heterogeneous Example .................... 64

3.3.2 A Synthetic Field Example ................................................................ 71

3.3.3 Measurements-Based Optimization (MBO): 2D Heterogeneous Case ........ 83

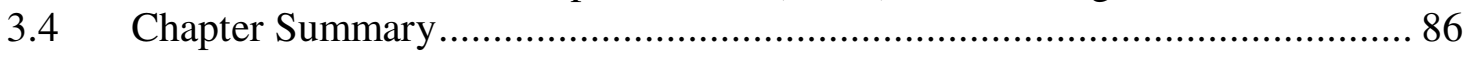

CHAPTER IV APPLICATIONS OF WATERFLOOD OPTIMIZATION VIA OPTIMAL RATE CONTROL WITH SMART WELLS ..................... 88

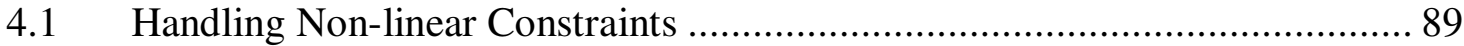

4.1.1 The Minimum Allowable Flowing Bottom-Hole Pressure (FBHHP) (Pwmin) ....................................................................................... 89

4.1.2 Rate Restriction on High Water Cut Well. .......................................... 93

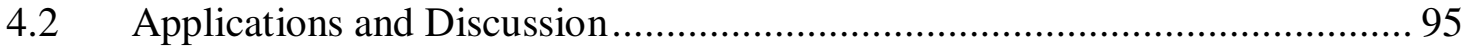

4.2.1 A Synthetic Field Example: Brugge Field ............................................ 95

4.2.2 Field Application in Giant Middle-Eastern Oil Field ............................... 110

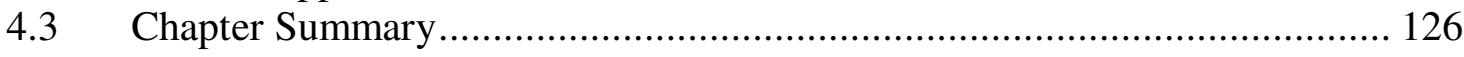

CHAPTER V CONCLUSIONS AND RECOMMENDATIONS ........................... 127

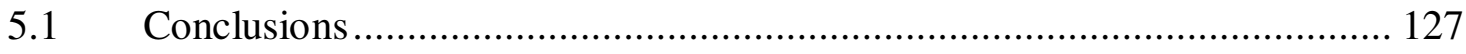

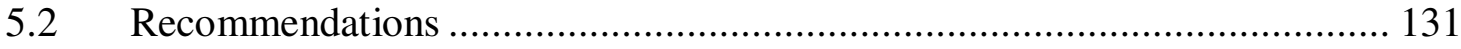

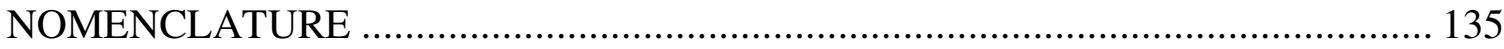

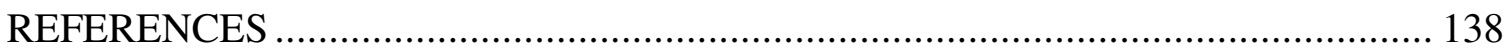

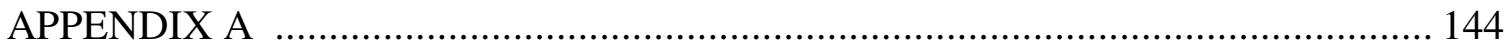

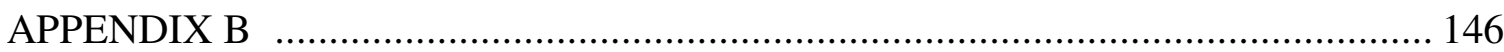


APPENDIX C

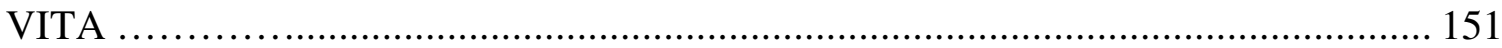




\section{LIST OF FIGURES}

Page

Figure 2.1 Well locations: 2D homogenous example ..................................... 10

Figure 2.2 Relative permeability curves...................................................... 11

Figure 2.3 Arrival time and water cut prior to rate optimization: 2D homogenous

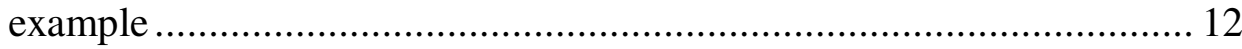

Figure 2.4 Arrival time and water cut after rate optimization: 2D homogenous example .......................................................................... 14

Figure 2.5 Oil saturation at arrival time: 2D homogenous example .................... 15

Figure 2.6 Comparison before and after rate optimization in terms of water cut and cumulative water production: 2D homogenous example ............... 16

Figure 2.7 Permeability field: 2D heterogeneous example ............................ 17

Figure 2.8 Water arrival time prior to rate optimization .................................. 18

Figure 2.9 Water arrival time after rate optimization........................................ 18

Figure 2.10 Total production/injection rates after rate optimization, $M=1: 2 \mathrm{D}$ heterogeneous example......................................................... 19

Figure 2.11 Oil saturation at arrival time: 2D heterogeneous example.................... 20

Figure $2.12 \quad$ Streamlines: 2D homogenous example...................................... 30

Figure 2.13 Comparison between analytical and numerical sensitivities................. 32

Figure 2.14 Production/injection rates after rate optimization, $M=0.25: 2 \mathrm{D}$ heterogeneous example ................................................................ 34

Figure 2.15 Production/injection rates after rate optimization, $M=10: 2 \mathrm{D}$ heterogeneous example ............................................................ 35

Figure 2.16 2D example for smart wells optimization ................................... 37

Figure 2.17 Oil saturation at arrival time: 2D smart well example........................ 37 
Figure 2.18 Water cut profile for 50 segments along the horizontal section: smart well example 39

Figure 2.19 Total fluid production and injection after rate optimization: smart well example

Figure 2.20 Porosity and permeability distribution: field example.

Figure 2. 21 Wells groups at the first and second time-steps for the field example. (inactive wells are shown in grey) 43

Figure 2.22 Field water injection before and after optimization acknowledging field constraints 44

Figure 2.23 Performance of 33 wells after optimization: field example................... 45

Figure 2.24 Performance plots for the field example ..................................... 46

Figure 2.25 Difference maps in terms of oil saturation between the base case and the optimized case (So, base - So, optimized) @ 5000 days: field example 47

Figure 2.26 Performance plots for P28 and P31 : field example ......................... 48

Figure 3.1 Flow chart to show the major step in the optimization approach........... 53

Figure 3.2 Well locations: 2D heterogeneous case ...................................... 64

Figure 3.3 Multiple realizations of permeability (md): 2D heterogeneous case....... 65

Figure 3.4 Optimized rates using different number of realizations (Nr): 2D heterogeneous case. 66

Figure 3.5 Comparison between the base case and the optimized case in terms of expected value and standard deviation: 2D heterogeneous case, $\mathrm{Nr}=32$

Figure 3.6 Comparison in terms of the expected value for different $r$ and the worst case scenario after optimization: 2D heterogeneous case, $\mathrm{Nr}=32$. 
Figure 3.7 Comparison in terms of the standard deviation for different $r$ and the worst case scenario after optimization: 2D heterogeneous case, $\mathrm{Nr}=32$

Figure 3.8 Comparison in terms of the maximum of the square of $l_{2}$ norm of residuals for different $r$ and the worst case scenario after optimization : 2D heterogeneous case, $\mathrm{Nr}=32$

Figure 3.9 Comparison between the optimized case using 32 realizations and no control case in terms of the average cumulative oil production from 150 realizations : 2D heterogeneous case, $r=0$

Figure 3.10 Comparison between the optimized case using 32 realization, the optimized case using a single realization, and the base case in terms of the average cumulative oil production from 150 realizations: $2 \mathrm{D}$

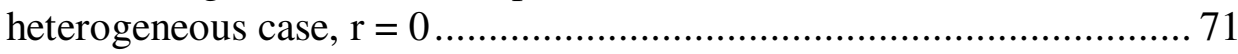

Figure 3.11 Well locations: 3D synthetic field case......................................... 72

Figure 3.12 Porosity and permeability fields for different layers: 3D synthetic field case

Figure 3.13 Average porosity and permeability per layers: 3D synthetic field case .. 73

Figure 3.14 Integrated map showing mainly fracture distribution and orientations after Cosentino et al. (2002).

Figure 3.15 Discrete fracture network maps on the top and the corresponding gridbased fracture permeability maps below: 3D synthetic field case

Figure 3.16 Smart-complex well completions, 2 ICVs along the mother-bore (L1 and L2) and one ICV at the entry of each lateral (L3 and L4): $3 \mathrm{D}$ synthetic field case 75

Figure 3.17 Comparison between the base case and the optimized case in terms of the total fluid production: 3D synthetic field case 76

Figure 3.18 Comparison between the base case and the optimized case in terms of the total fluid injection: 3D synthetic field case.

Figure3. 19 Bubble map showing rate allocations for the base case, the size of the bubble represents the amount of total injection/production rate: $3 \mathrm{D}$ synthetic field case. 
Figure 3.20 Bubble map showing fate allocations for the optimized case, the size of the bubble represents the amount of total injection/production rate: $3 \mathrm{D}$ synthetic field case ..................................................... 80

Figure 3.21 Normalized objective function evaluation per iteration: 3D synthetic field case

Figure 3.22 Comparison between the base case and the optimized case in terms of water cut: 3D synthetic field case.

Figure 3.23 Cumulative oil production ratio vs. time: 3D synthetic field case ......... 82

Figure 3.24 Cumulative water production ratio vs. time: 3D synthetic field case..... 82

Figure 3.25 True permeability field: MBO..................................................... 83

Figure 3.26 Water saturation map for the true model after 200 Days: MBO........... 84

Figure 3.27 Calculated weight for 32 realizations using Bayes' Theorem: MBO..... 85

Figure 3.28 Comparison between the MBO and optimize case using equal weights in terms of total production rate: $\mathrm{MBO}$............................. 85

Figure 3.29 Comparison between the MBO, optimize case using equal weights, and the base case in terms of water cut vs. oil recovery: MBO .............86

Figure 4.1 Cross plot to compare wells block pressures between multiple iterations within a single optimization time step.............................. 91

Figure 4.2 Cross plot to compare well rates between multiple iterations within a single optimization time step .................................... 92

Figure 4.3 Cross plot to compare well water cut between multiple iterations within a single optimization time step: the water cut is normalized to the highest water cut ........................................................... 94

Figure 4.4 Map showing the structure of Brugge field and wells locations ............. 96

Figure 4.5 Water cut misfit and generalized travel time misfit .......................... 98

Figure 4.6 Permeability changes after history matching ................................ 99 
Figure 4.7 History matching results for the field overall production: Brugge field .......

Figure 4.8 Comparisons between observed, initial, and matched water cut for multiple wells: Brugge field.

Figure 4.9 Comparisons between observed, initial, and matched FBHP for multiple wells: Brugge field.....

Figure 4.10 Comparison between the base case and the optimized case in terms of average cumulative oil and water productions: Brugge field, scenario 1

Figure 4.11 Comparison between the base case and the optimized case in terms of average cumulative oil and water productions from 20 realizations:

Brugge field, scenario 2 108

Figure 4.12 Comparison between scenario 1 and scenario 2 in terms of resevoir pressure and total field productin rate: Brugge field

Figure 4.13 BR-P-1 rate performance over 20 years for scenario 1 and 2: Brugge field

Figure 4.14 Comparisons between the base case and the optimized case in terms of oil saturation for layer 4 and 5: Brugge field

Figure 4.15 Streamlines configuration, area of interest is within the white box: giant field example

Figure 4.16 Injectors groups and the corresponding producers groups: giant field example; some of the groups are hidden per field operator request...... 116

Figure 4.17 Well locations, area of interest: giant field example

Figure 4.18 Time of flight in the area of interest, Smart MRC well location and completion: giant field example

Figure 4.19 Comparison between the base and the optimized case in terms of cumulative water injection, oil production, and water production; $\mathrm{y}$-axis scale is removed per operator request 
Figure 4.20 Bubble maps which compare the base and the optimized cases in terms of production rates and injection rates per well at the first time step: giant field example ................................................. 121

Figure 4.21 Comparison between the base and optimized cases for the smart MRC well in terms of oil and water rates and oil and water cumulative: giant field example

Figure 4.22 Comparisons between the base and the optimized cases in terms of the FBHP for two wells located in the core area: giant field example

Figure 5.1 Flow chart to show the optimization process at multiple time steps .... 129 


\section{LIST OF TABLES}

Page

Table 2.1 Production rates prior to and after optimization: 2D homogenous

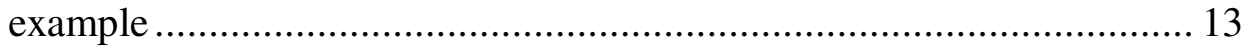

Table 2.2 Sensitivity of arrival time at producers with respect to production

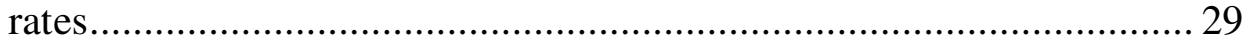

Table 2.3 Sensitivity of arrival time at producers with respect toinjection rates .... 31 


\section{CHAPTER I}

\section{INTRODUCTION}

The recent increase in worldwide oil demand combined with the decreasing number of new discoveries has underscored the need to efficiently produce existing oil fields. The maturity of the existing fields poses multifaceted challenge to meet the global demand and has increased the urgency to seek new alternatives to increase production and improve recovery. A variety of secondary oil recovery methods has been developed to improve oil recovery after primary depletion (Lake et al. 1992; Craig 1971). The most widely used is waterflooding because it is relatively easy and inexpensive to implement. In spite of its many appealing characteristics, the presence of heterogeneity such as high permeability streaks might yield unfavorable results (Sudaryanto and Yortsos 2001; Brouwer et al. 2001; Brouwer and Jasen 2004; Alhuthali et al. 2007). Those streaks can act as highly conductive channels causing injected water to bypass oil and prematurely breakthrough at the producers. This will result in a poor sweep ifficiencyand consequently reduce oil production and recovery. Various methods have been suggested to mitigate this problem. Among these is smart well completions where the production or the injection section is divided into several intervals (Arenas and Dolle 2003; Glandt 2003; Hussain et al. 2005). The flow rate at each interval can be independently controlled by inflow control valves (ICVs); hence, making it possible to control flow rates across the high permeability streaks. The smart well technology has several advantages including the following: 1) access to multiple zones using only one well, 2) cost savings by minimizing well down-time and intervention, and 3) quick response to a sudden change in well performance.

The appealing features of the smart well technology have inspired several researchers to develop efficient algorithms to optimize production along the intervals of

This dissertation follows the style of SPE Reservoir Evaluation \& Engineering. 
smart wells, and thereby improve sweep efficiency. Two main types of optimization algorithms were developed, namely the gradient-based algorithms and the stochastic algorithms (Brouwer and Jasen 2004; Sarma et al. 2005; Tavakkolian et al. 2004; Emerick et al. 2007). Both algorithms use reservoir simulators to evaluate the objective function. The gradient-based algorithms require an efficient estimation of the gradient of the objective function with respect to the control variables. In contrast, the stochastic algorithms such as genetic algorithms do not require an estimation of the gradient, but they require multiple forward simulation runs to find the global minimum. The advantage of stochastic optimization over the gradient-based optimization is the ability to search for a global solution while the gradient-based optimization generally converges to a local solution. The main disadvantage of the stochastic optimization is the extensive computation power required especially when the number of control variables is large.

Field scale rate optimization problems generally involve highly complex reservoir models, production and facilities related constraints and a large number of unknowns. All these make optimal reservoir management via rate and flood front control difficult without efficient optimization tools. Some aspects of the optimization problem have been studied before using mainly optimal control theory. However, the applications to-date have been limited to rather small problems because of the computation time and the complexities associated with the formulation and solution of adjoint equations. Fieldscale rate optimization for maximizing waterflood sweep efficiency under realistic field conditions has still remained largely unexplored.

The purpose of this research is three-fold. First, we develop a practical and efficient approach for computing optimal injection and production rates to maximize sweep efficiency and delay the water arrival time to minimize water cycling. Our work will rely on equalizing the arrival time of the waterfront at all producers within selected sub-regions of a water flood project. The arrival time optimization has favorable quasilinear properties and the optimization proceeds smoothly even if our initial conditions are far from the solution (Cheng el al. 2005). Furthermore, the sensitivity of the arrival time with respect to injection and production rates can be calculated analytically using a 
single flow simulation. This makes our approach computationally efficient and suitable for large-scale field applications. The arrival time optimization ensures appropriate rate allocation and flood front management by delaying the water breakthrough at the producing wells. Second, the approach will be generalized to account for geologic uncertainty since reservoir parameters such as permeability are known in a stochastic sense. The uncertainty quantification will be addressed by performing the optimization on multiple permeability realizations. Finally, we will account for reservoir and facility constraints through a constrained non-linear optimization viz. sequential quadratic programming (SQP).

\subsection{Optimal Waterflood Management Using Rate Controls}

Previous efforts on optimization of waterflooding relied on optimal control theorem to allocate injection/production rates for fixed well configurations. Asheim (1988) investigated the optimization of waterflood based on maximizing net present value (NPV) for multiple vertical injectors and one producer where the rate profiles change throughout the optimization time. Sudaryanto and Yortsos (2001) used maximizing the displacement efficiency at water breakthrough as the objective for the optimization with two injectors and one producer. The optimal injection policy was found to be 'bang bang' type. That is, the injectors were operated only at their extreme values, either at the maximum allowable injection rate or fully shut. The optimization then involved finding the switch time between the two injectors to ensure simultaneous water arrival at the producing well. Brouwer et al. (2001) studied the static optimization of waterflooding with two horizontal smart wells containing permanent downhole well control valves and measurement equipment. The static optimization implies that the flow rates of the inflow control valves (ICVs) along the well segments were kept constant during the waterflooding process until the water arrived at the producer. Various heuristic algorithms were utilized to minimize the impact of high permeability streaks on the waterflood performance through rate control. The results indicated that the optimal rate allocation amounts to reducing the distribution of water arrival times at various 
segments along the producer. Subsequently, Brouwer and Jansen (2004) extended their work to dynamic optimization of water flooding with smart wells using optimal control theory. The optimization was performed on one horizontal producer and one horizontal injector. Each well is equipped with 45 ICVs. The objective was to maximize the NPV, and it was achieved through changing the rate profile along the well segments throughout the optimization period. Both rate constrained and bottomhole pressure constrained well conditions were studied.

Lorentzen et al. (2006) also carried out a study on the dynamic optimization of waterflooding using a different approach from those described above. He carried out his optimization by controlling the chokes to maximize cumulative oil production or net present value. Their new approach uses the ensemble Kalman filter as an optimization routine. The ensemble Kalman filter was originally used for estimation of state variables but has been adapted to optimization in their work.

Several authors have reported the use of streamlines to optimize waterflood projects. Grinestaff and Caffrey (2000) provided detailed case histories of waterflood optimization for the Northwest Fault Block (NWFB) of Prudhoe Bay. They didn't use a formal optimization procedure, but instead they used streamline flow visualization, allocation calculations, and the rapid turnaround of simulation runs to guide the engineer. They utilized allocation factors (Grinestaff 1999) as their primary criterion for optimal sweep. Thiele and Batycky (2006) provide a different measure of flood efficiency. Their technique focuses mainly on the injectors and calculates the efficiency for each to identify the least effective injectors. The injection capacity can then be reallocated to improve the waterflood performance. Ghori et al. (2007) used the injection efficiency optimization to generate injection rate to improve waterfood performance in a giant Middle East field. The optimization procedures were constrained to a realistic field conditions to ensure direct field implementation. 


\subsection{Optimal Waterflood Management under Geologic Uncertainty}

To address the problem of geologic uncertainty in production optimization, two approaches have been studied. The first one is based on a closed-loop approach where measurements from smart wells were used to continuously update the reservoir model and an optimal control strategy was used to allocate rates based on the most recent update of the reservoir model. Naevadal et al. (2006) developed a closed-Loop control approach which is a combination of an optimal control for waterflood optimization and automatic history matching using ensemble Kalman filter for reservoir model updating. They observed that the results obtained using a closed-loop approach starting from an unknown permeability field, were almost as good as those obtained assuming a proiri knowledge of the permeability field. A similar closed-loop approach was adapted by Sarma et al. (2005). However, in their work, they used Karhunen-Loeve (K-L) expansions and Bayesian inversion theory for history matching and model updating. Wang et al. (2007) used another closed loop approach which is still a combination of history matching and rate control optimization. They used ensemble Kalman filter for automatic history matching and real time model updating. For rate control optimization, they compared the performance of three different methods namely steepest ascent, simultaneous perturbation stochastic approximation (SPSA), and ensemble Kalman filter. Their result showed that the steepest ascent algorithm is the most efficient and gives reasonable results.

The second type of handling of uncertainty is to perform the optimization over multiple realizations since the reservoir geology is only known in a probabilistic framework. Van Essen et al. (2006) expanded on the work done by Brouwers et al (2004) and wrote the objective function in terms of the expected value of NPV obtained from multiple realizations. They used the adjoint method to compute the gradient of the objective function and the steepest ascent algorithm to maximize it. Their results showed that their optimization approach improve the expected NPV and resulted in smaller variance of possible NPV outcomes. Similarly, Chen et al. 2008 performed the optimization over multiple realizations to maximize the expected NPV, however they 
used ensemble Kalman filter to compute the gradient of the objective function. Their method includes a model updating part. They used the ensemble Kalman filter for model updating (Gu and Oliver 2007).

\subsection{Objectives}

The main objective of this research is to develop an efficient and practical optimization approach to compute injection and production rates in a waterflooding project to maximize sweep efficiency. The approach should have the ability to handle geologic uncertainty, large field cases, and operational and facility constraints.

\subsubsection{Optimal Waterflood Management for a Single Reservoir Model}

- The first step in our research is to develop the objective function for our optimization which will address the sweep efficiency for a single reservoir model. The principle concept behind our optimization is to equalize the arrival at all producers within a sub-group of wells. The arrival time optimization was chosen because of their quasi-linear property and it also ensures maximizing sweep efficiency. The objective function will be formulated as the square of $l_{2}$ norm of the residuals between a desired arrival time and a calculated arrival time

- The objective function then will be minimized using a sequential quadratic programming algorithm. This algorithm will minimize the objective function in an iterative manner. In each iteration, the algorithm requires evaluating the following: 1) the objective function and 2) the gradient and the Hessian of the objective function.

- The objective function evaluation will involve an analytical calculation of the residuals using the concept of time of flight. Of course, this step can't be accomplished unless we trace the streamlines.

- The gradient and the Hessian of the objective function will be computed analytically using an analytical form for the sensitivity of the arrival time with 
respect to the control variables (i.e. wells rates). The analytical computation of the gradient and Hessian will require only one simulation run.

\subsubsection{Optimal Waterflood Management under Geological Uncertainty}

- To address geologic uncertainty, the optimization will be performed on multiple realizations. The objective function will be formulated into two forms: stochastic and min-max problem forms.

- The stochastic form will be derived based on the decision analysis framework. The objective function will involve an expected value of the $l_{2}$ norm of the residuals for each realization and their standard deviation. The expected value and the standard deviation will be combined through a risk coefficient.

- The min-max problem will be formulated as minimizing the maximum of outcomes evaluated for each realization involved in the optimization. This type of optimization can be considered as minimizing the worst case scenario.

- The gradient and the Hessian of the objective will be computed analytically using only one simulation run per realization. This will make our approach efficient and suitable for large field cases.

\subsubsection{Applications}

- We will show that our proposed approach is capable of optimizing injection/production rate under changing mobility effects and changing field conditions.

- We will demonstrate the ability of our proposed approach to efficiently allocate rates along the producing section of smart-complex wells.

- We will illustrate the practicality and efficiency of our proposed approach through field examples. 


\section{CHAPTER II}

\section{OPTIMAL WATERFLOOD MANAGEMENT VIA RATE CONTROL USING SINGLE RESERVOIR MODEL ${ }^{*}$}

In this chapter we propose an optimal reservoir management scheme during waterflooding using a single reservoir model. The scheme relies on an appropriate allocation of injection and production rates to maximize the sweep efficiency. The underlying principle behind our optimization scheme is to equalize the arrival time of the waterfront at all producers within selected sub-regions of a water flood project. The organization of this chapter is as follows. First, we outline the major steps of the proposed approach and illustrate the procedure using a 2D synthetic example. Next, we discuss the underlying mathematical formulation. These include the optimization problem, residual computations, the analytical computation of the sensitivities, and field and individual well constraints. We also examine the convergence properties of our method and the validity of the assumptions used to derive the analytical sensitivities. Finally, we demonstrate the power and feasibility of our approach using multiple 2D examples at various mobility ratios and a 3-D field case.

\subsection{Approach}

Our approach can be implemented in both finite-difference and streamline simulators. In this study, we chose to use a commercial streamline simulator because the streamlines and the time of flight (which are essential for our optimization) are readily available (ECLIPSE File Formats Reference Manual 2005). If a finite-difference simulator is used instead, then we need to perform the streamline tracing and time of

\footnotetext{
* Part of this chapter is reprinted with permission from "Optimal Waterflood Management Using Rate Control" by Ahmed H. Alhuthali, Dayo Oyerinde, and Akhil Datta-Gupta, 2007. SPE Reservoir Evaluation \& Engineering, 10 (5):539-551, Copyright 2007 by the Society of Petroleum Engineers.
} 
flight computations using the total phase fluxes from the simulator (Cheng et al. 2005). In addition, we assume that a static model is readily available and the injected fluid composition is fixed and it is not a control variable. Our optimization scheme is limited only to optimize wells rates and it doesn't include well location and configuration as control variables to improve sweep. The major steps in our approach are outlined below.

- Tracing streamlines and arrival time computation. The first step is to generate streamlines and compute the time of flight. Tracing streamlines has been discussed by several authors (King and Datta-Gupta 1998; Datta-Gupta and King 1995; Pollock 1988). The time of flight, which is defined as the time required by a neutral tracer particle to travel along a streamline will form the basis for computing the arrival time of the waterfront at all producers. If a streamline simulator is being used, all these quantities are already available and this step can be bypassed.

- Residuals and Sensitivity Computation. In this step we compute the residuals that quantify the difference between the desired arrival time and the computed arrival time at each of the producing well. We also calculate the sensitivity of the arrival time at the producer to wells rates analytically using simple integrals along streamlines. The sensitivities are partial derivatives that relate changes in arrival time to small perturbations in production and injection rates. They are an integral part of our rate optimization process.

- Minimization and Optimal Rate Allocations. Sequential quadratic programming (SQP) procedures (Nocedal and Wright 2006) is used to minimize the arrival time residuals. This step generates the required changes in rates to equalize waterfront arrival time at the producing wells subject to appropriate field constraints.

- Mobility Effects and Changing Field Conditions. The steps above are repeated until the norm of the residuals meets a pre-defined stopping criterion. Once this criterion is met, we move to a new time interval, update streamlines 
and perform the optimization again to account for mobility effects and changing field conditions.

\subsection{An Illustration of the Procedure}

The detailed mathematical formulation behind our proposed approach will be discussed later. First, we will illustrate the procedure using a 2D example that involves four injectors and nine producers arranged in a repeated five-spot pattern (Figure 2.1).

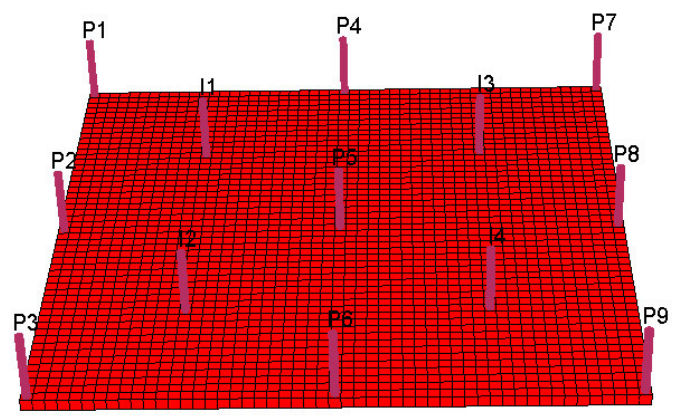

\section{Figure 2.1 Well locations: 2D homogenous example}

We assume homogenous permeability $(\mathrm{k}=100 \mathrm{md})$ and an end-point mobility ratio of unity for this example. The relative permeability curves are shown in Figure 2.2. The reservoir dimensions are $1716 \times 1716 \times 33 \mathrm{ft}$, and the porosity is assumed to be constant and equals to 0.225 . As a base case, we choose the total fluid production rate to be the same for all producers (280 RB/D) and also, for all injectors (630 RB/D). The optimization was performed simultaneously on both the injectors and the producers to equalize the arrival times of the injected fluid at all producers. 


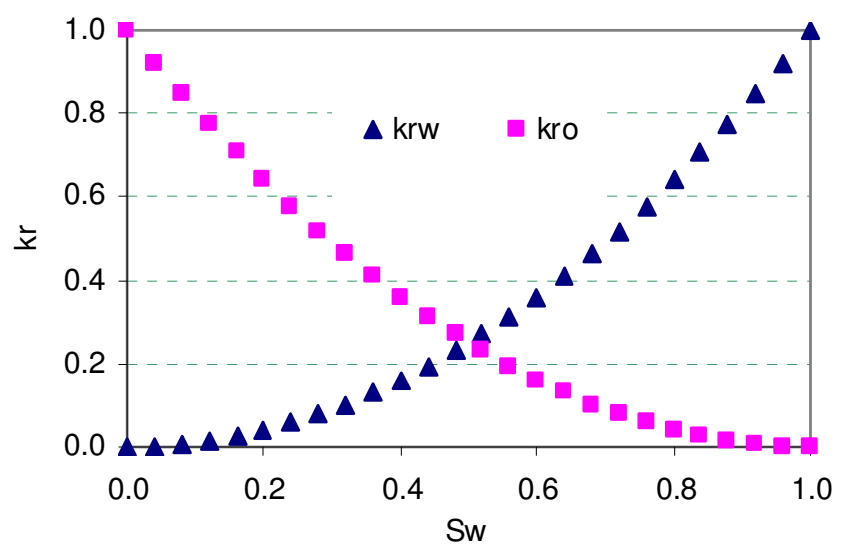

\section{Figure 2.2 Relative permeability curves}

The arrival time distribution at the producing wells and their water cut response for the base case are shown in Figures 2.3a and 3b.

Clearly, there is a significant variation in arrival times whereby some producers reach high water cut rapidly compared to others. This is a consequence of assigning equal rates to all producers regardless of their location. For example, the well P5 is located in the middle and its delayed breakthrough can be explained by the fact that it is supported by four injectors, each contributing to a quarter of the production. In contrast, the early water breakthrough at the corner producers (P1, P3, P7, and P9) is because each producer here is supported by only one injector, contributing to all of the production. The water breakthrough for the side producers ( $\mathrm{P} 2, \mathrm{P} 4, \mathrm{P} 6$, and P8) is inbetween because each producer is supported by two injectors. Next, the production rates were reassigned to equalize the arrival time. 


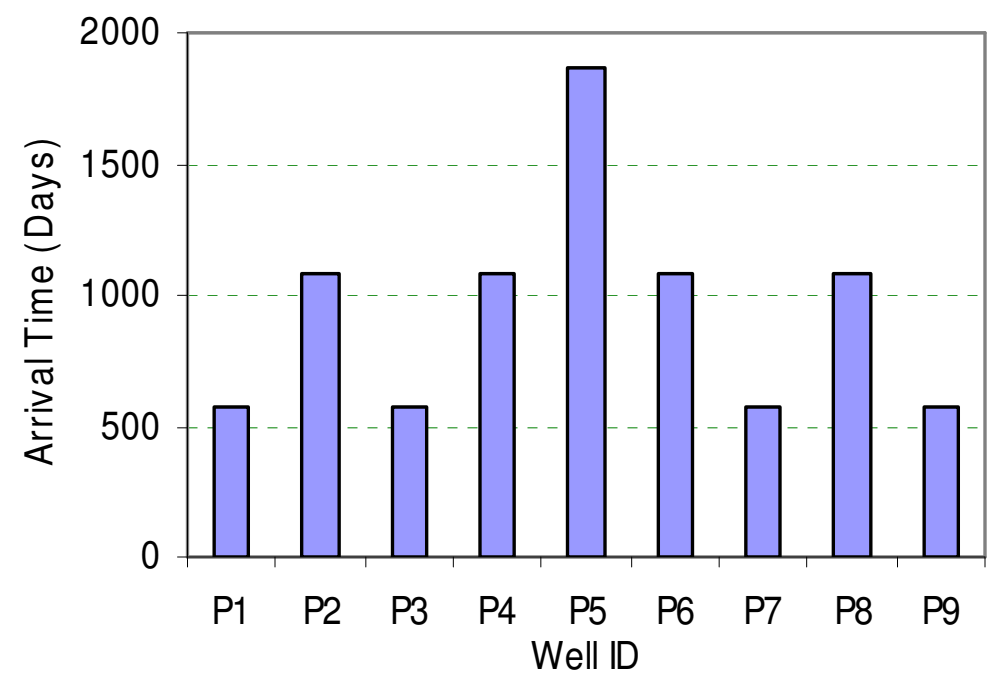

a. Water arrival time

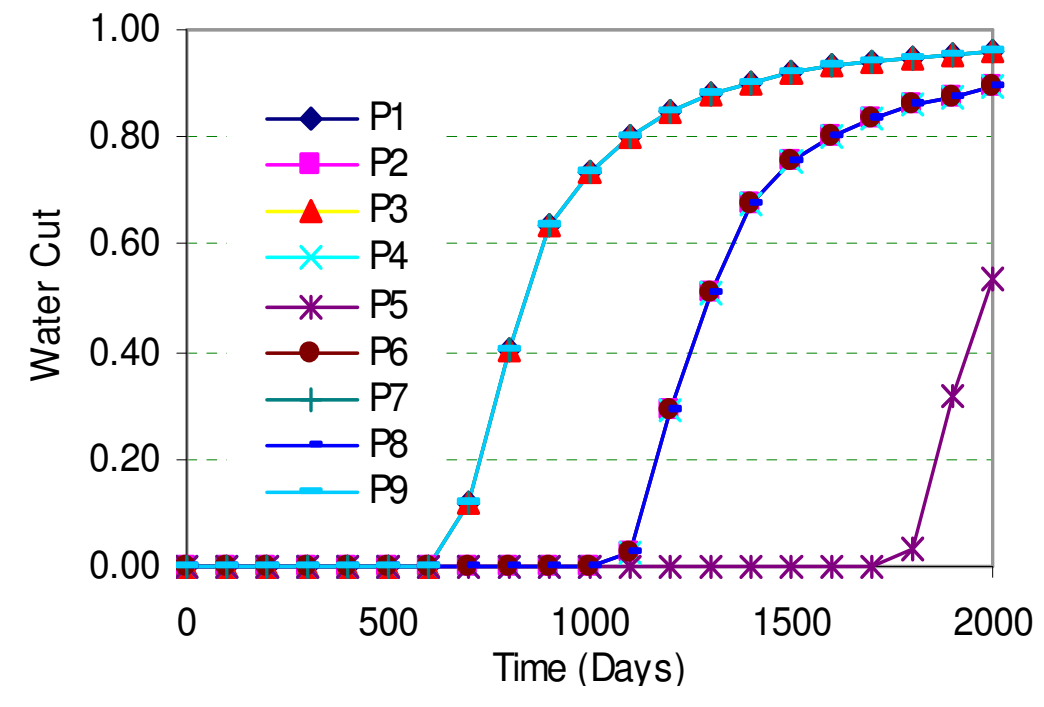

b. Water cut

Figure 2.3 Arrival time and water cut prior to rate optimization: 2D homogenous example 
The static optimization was performed under voidage replacement constraint, that is, the total field production is equal to the field injection. The new rates after optimization are shown in Table 2.1. There were no changes to the injection rates, and significant changes to the production rates.

Table 2.1 Production rates prior to and after optimization: 2D homogenous example

\begin{tabular}{|c|c|c|c|}
\hline $\begin{array}{c}\text { Well } \\
\text { ID }\end{array}$ & $\begin{array}{c}\text { Rate Before } \\
\text { Optimization (RB/D) }\end{array}$ & $\begin{array}{c}\text { Rate After } \\
\text { Optimization (RB/D) }\end{array}$ & $\begin{array}{c}\text { Optimal Rate } \\
\text { Geometrical (RB/D) }\end{array}$ \\
\hline P1 & 280.0 & 157.7 & 157.5 \\
\hline P2 & 280.0 & 314.9 & 315.0 \\
\hline P3 & 280.0 & 157.7 & 157.5 \\
\hline P4 & 280.0 & 314.9 & 315.0 \\
\hline P5 & 280.0 & 629.6 & 630.0 \\
\hline P6 & 280.0 & 314.9 & 157.5 \\
\hline P7 & 280.0 & 157.7 & 315.0 \\
\hline P8 & 280.0 & 314.9 & 157.5 \\
\hline P9 & 280.0 & 157.7 & 630.0 \\
\hline I1 & 630.0 & 630.0 & 630.0 \\
\hline I2 & 630.0 & 630.0 & 630.0 \\
\hline I3 & 630.0 & 630.0 & 630.0 \\
\hline I4 & 630.0 & 630.0 & \\
\hline
\end{tabular}

These changes lead to an equalized arrival times and excellent water cut performance as shown in Figs. 2.4a and 4b. The results agree with optimal rates calculated from simple geometrical analysis (angle open to flow) for this homogeneous 
example. Figures 2.5a and 5b show oil saturation at breakthrough prior to and after optimization. Increased sweep efficiency after optimization can be clearly seen here.

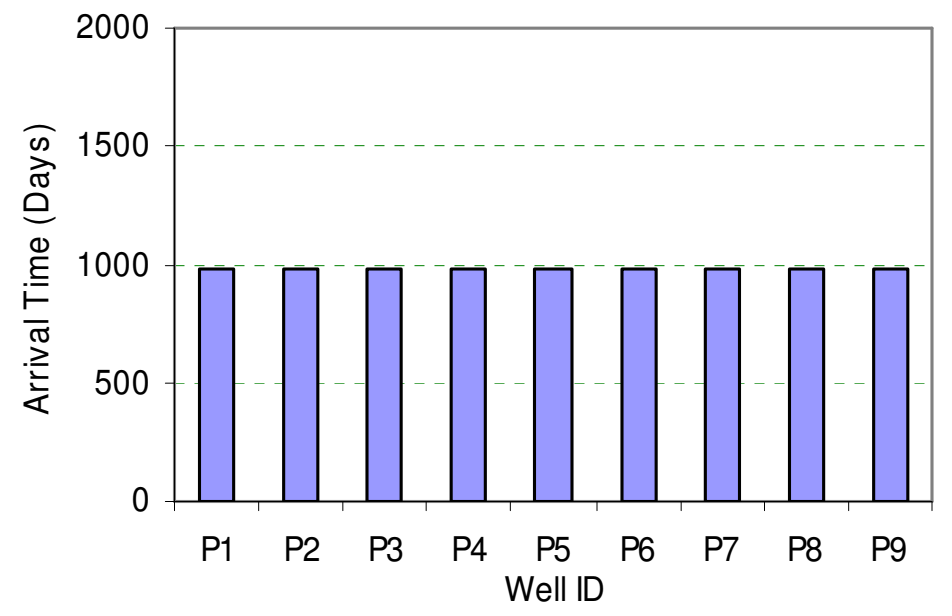

4a. Wells arrival time

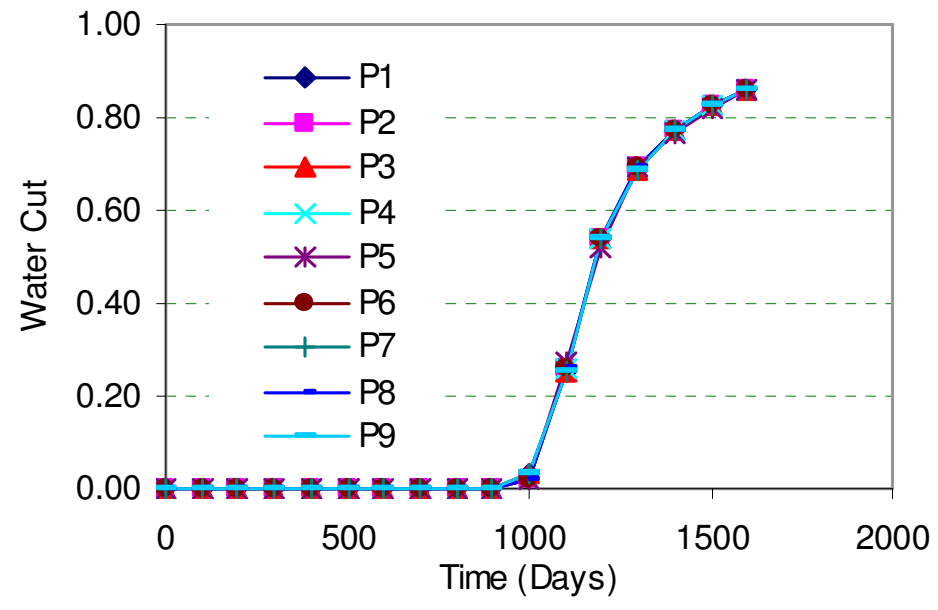

4b. Water cut

Figure 2.4 Arrival time and water cut after rate optimization: 2D homogenous example 


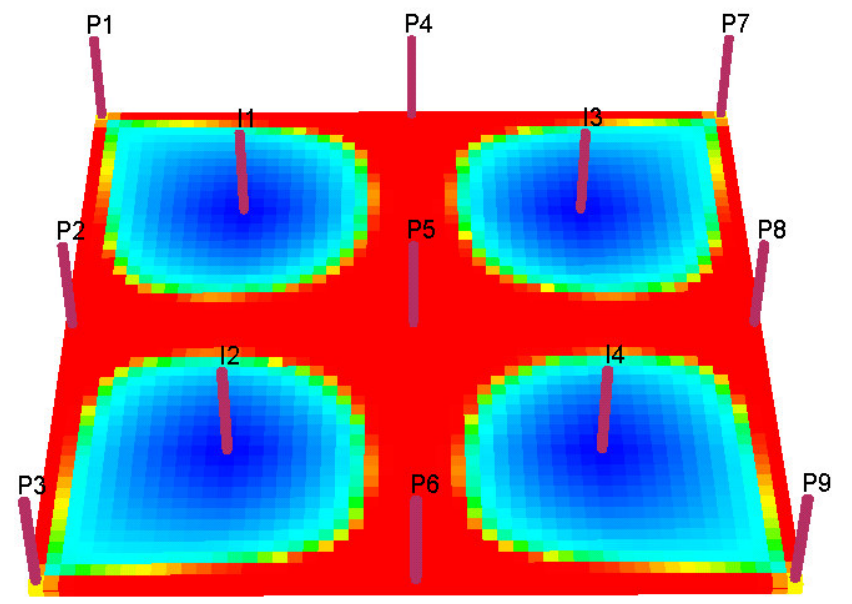

a. Prior to rate optimization, water arrives at 700 days

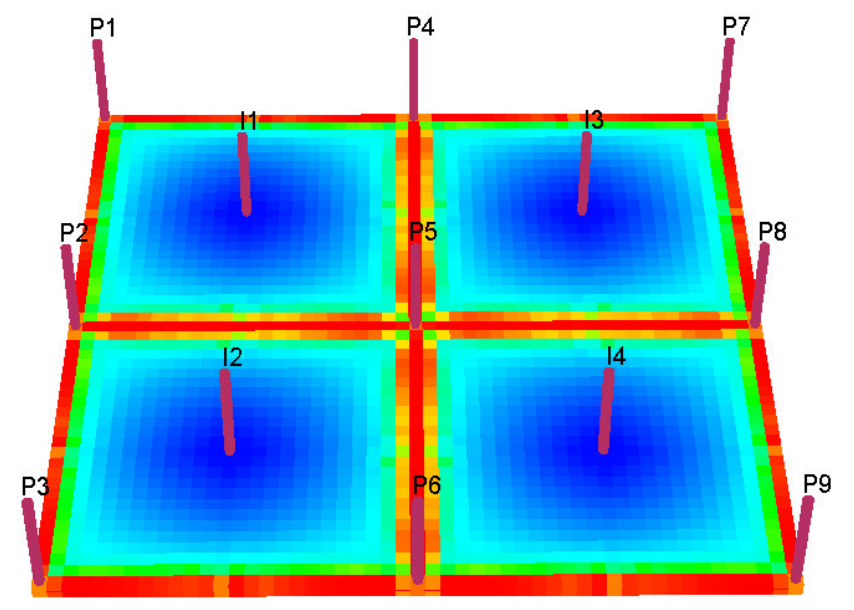

b. After rate optimization, water arrives at 1000 days

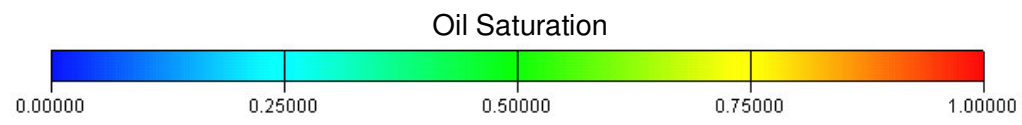

Figure 2.5 Oil saturation at arrival time: 2D homogenous example 
Also, after optimization, water breakthrough occurs at $65 \%$ recovery as opposed breakthrough at $40 \%$ recovery without optimization (Figure 2.6a). Performance plots such as water cut vs. recovery and cumulative water production vs. time shown in Figures. 2.6a and 6b demonstrate improved waterflood characteristics, for example, higher recovery and reduced water cycling.

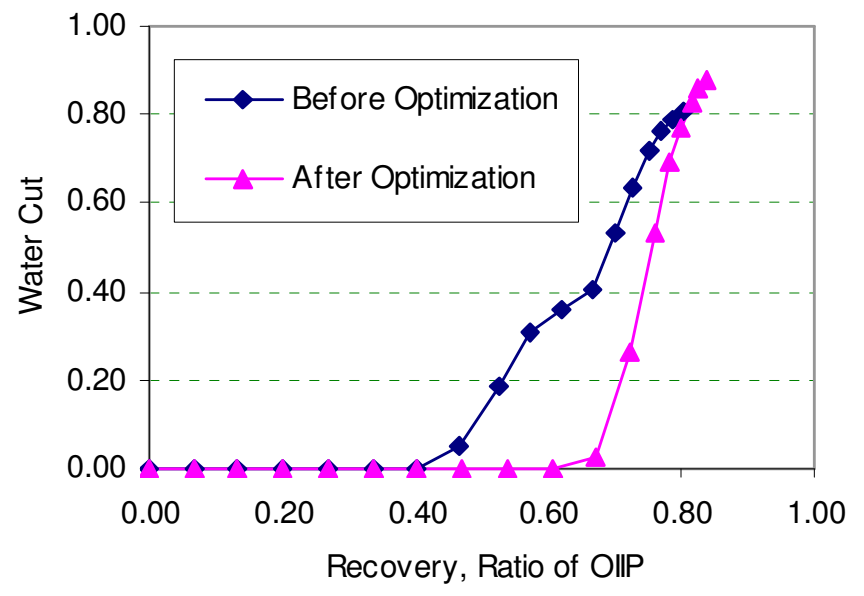

a. Water cut vs. recovery

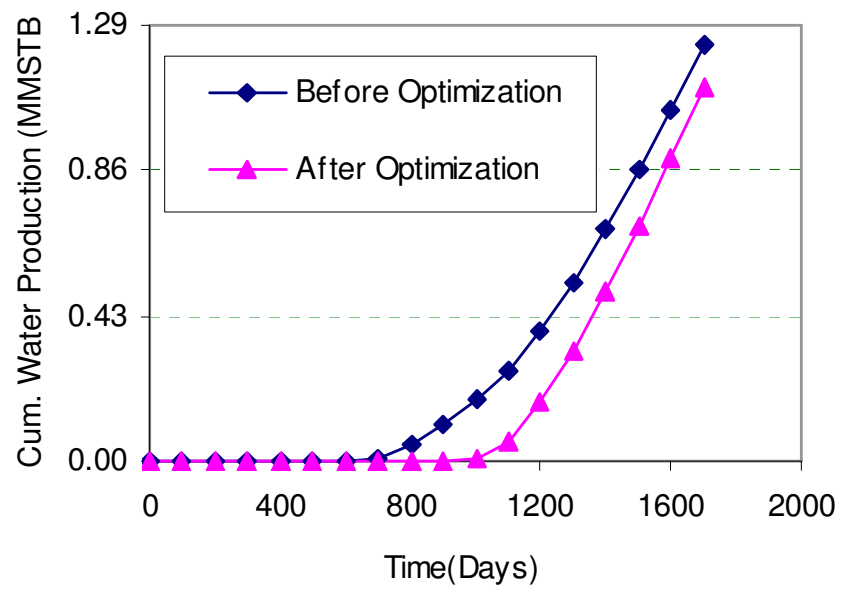

b. Cumulative water production vs. time

Figure 2.6 Comparison before and after rate optimization in terms of water cut and cumulative water production: $2 \mathrm{D}$ homogenous example 
Although this example was rather simple and the optimal rates could be obtained using a simple geometrical analysis, it serves to verify our approach. We now change the permeability field to a heterogeneous case as shown in Figure 2.7. The permeabilities range over three orders of magnitude and have a north east trend. Obviously, the optimization is less intuitive here to reach a simultaneous arrival time at all producers. For base case, we choose the optimized rates obtained from the homogenous case as our initial guess. This resulted in significant variation in arrival times as shown in Figure 2.8.

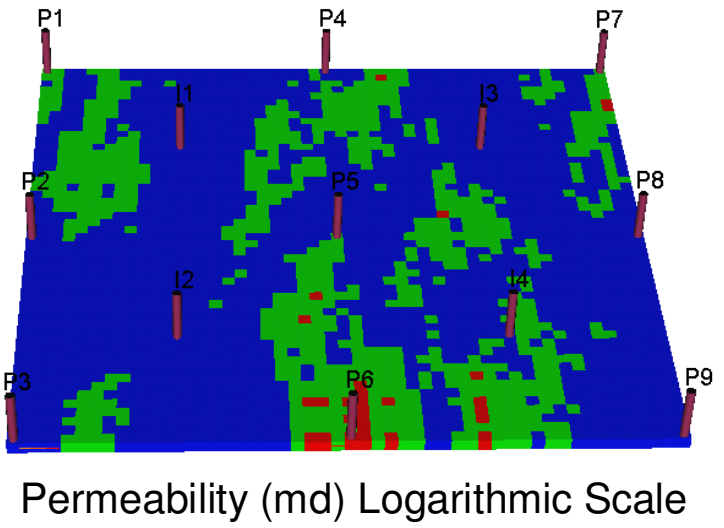

100

1000

10000

100000

Figure 2.7 Permeability field: 2D heterogeneous example 


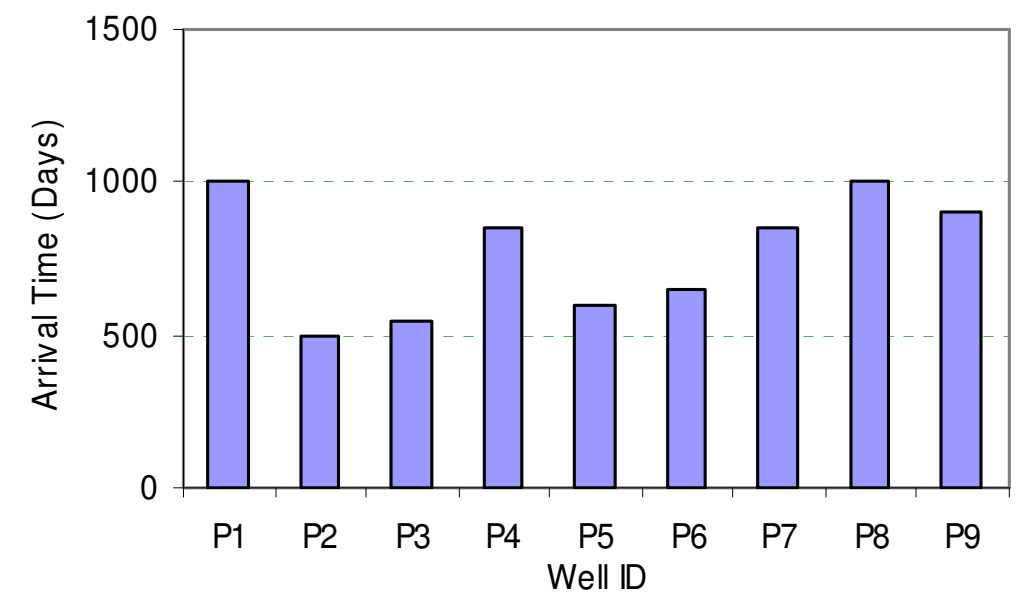

Figure 2.8 Water arrival time prior to rate optimization

After optimization, we managed to reduce this variation significantly, leading to a simultaneous arrival time of around 700 (Figure 2.9). The optimization was carried out at multiple time intervals: 100, 200, 300, 400, 500, 600, 700 days. Because the mobility ratio is chosen to be unity, very little variation in injection/production rates is observed with time as shown in Figs. 2.10a and 10b.

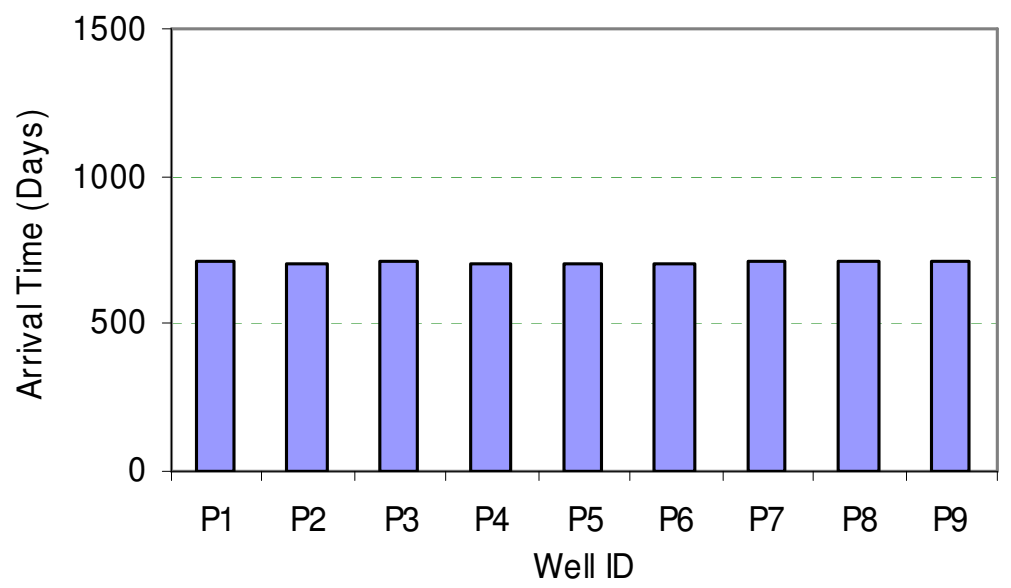

Figure 2.9 Water arrival time after rate optimization 


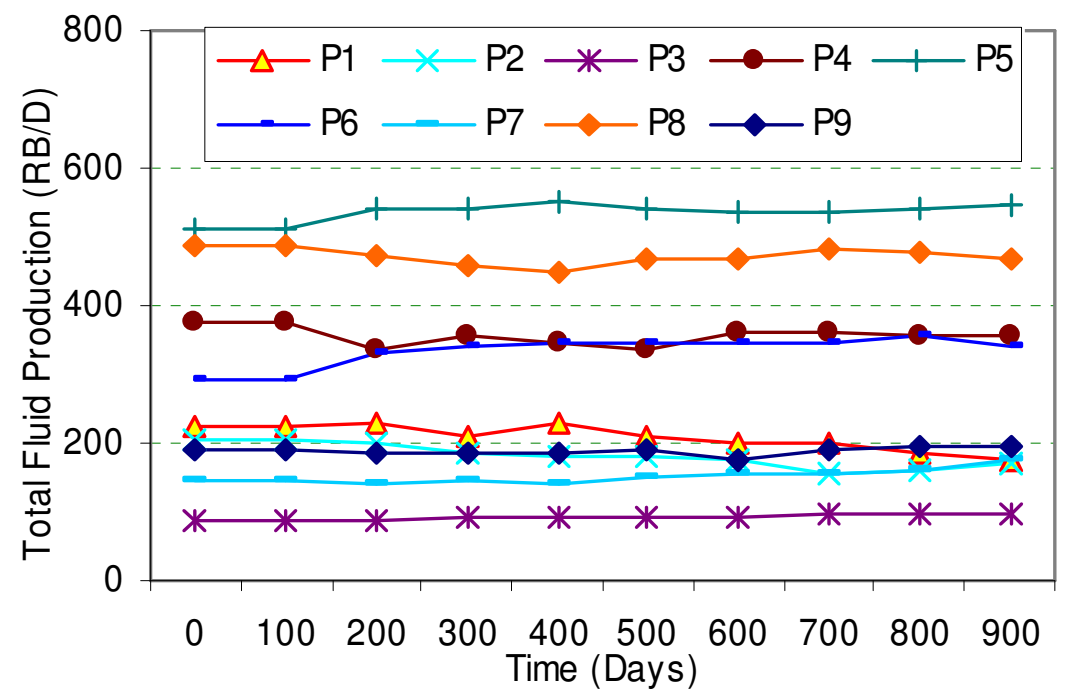

a. Total fluid rate

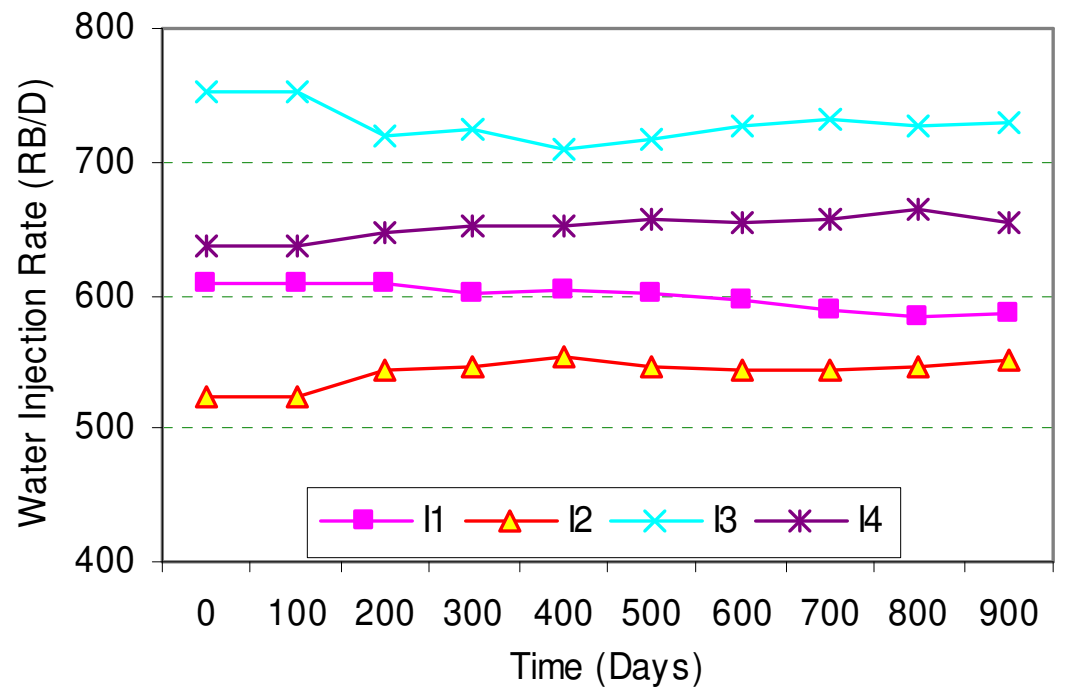

b. Water injection rate

Figure 2.10 Total production/injection rates after rate optimization, $M=1: 2 D$ heterogeneous example 
The optimized case show significant improvements in sweep efficiency and recovery at breakthrough as shown in Figure 2.11. The base case has a recovery of 37\% of OIIP while the optimized case has a recovery of $49 \%$ of OIIP, indicating the potential for improved recovery via rate optimization.

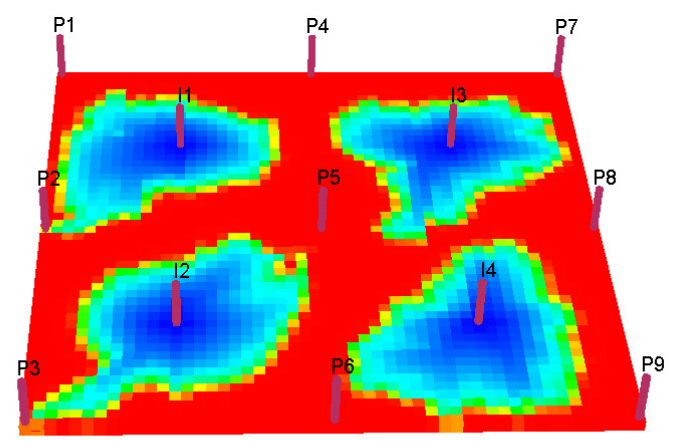

a. Prior to rate optimization, water arrival at $\mathbf{5 0 0}$ days, recovery $=\mathbf{3 7 \%}$ of OIIP

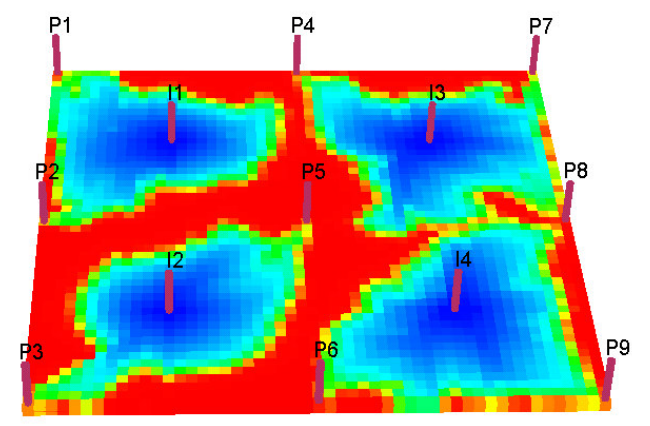

b. After rate optimization, water arrival at 700 days, recovery $=49 \%$ of OIIP

Oil Saturation

\begin{tabular}{|l|l|lr|rr|}
\hline & & & & \\
\hline 0.00000 & 0.25000 & 0.50000 & 0.75000 & 1.00000
\end{tabular}

Figure 2.11 Oil saturation at arrival time: 2D heterogeneous example 


\subsection{Mathematical Formulation}

In this section, we discuss the underlying mathematical formulations behind our proposed method. First, we present the formulation of the rate optimization based on waterfront arrival times and the streamline time of flight. We then discuss the analytical calculation of sensitivities of arrival time to well rates and their role in the optimization process. Finally, we briefly review field and individual well constraints in the optimization process.

\subsubsection{Objective Function and Its Minimization}

Our objective is to maximize the sweep efficiency by equalizing the arrival times of the waterfront for a specific group of producers (Sudaryanto and Yortsos 2001). Let $t_{i, m}$ denote the calculated arrival time for producer $i$ belonging to group $m$, and, $t_{d, m}$, the desired arrival time for the group $m$. Then the overall arrival time misfit can be expressed as.

$$
\|\mathbf{e}(\mathbf{q})\|_{2}^{2}=\sum_{m=1}^{N_{\text {group }}} \sum_{i=1}^{N_{\text {prod }, m}}\left(t_{d, m}-t_{i, m}(\mathbf{q})\right)^{2}
$$

where the arrival time residual at an individual well is given by

$$
e_{i, m}=t_{d, m}-t_{i, m}(\mathbf{q})
$$

The vector $\mathbf{q}$ contains the control variables (wells rate) and has a dimension of $\mathrm{n}$, the number of well rates to be optimized. Our goal is to minimize the misfit by optimizing the injection/production rates subject to equality and inequality constraints which are mainly defined by the operational restrictions and facility capacities. 
$\min _{\mathbf{q}} f(\mathbf{q})=\frac{1}{2}\|\mathbf{e}(\mathbf{q})\|_{2}^{2}$

Subject to

$$
\begin{aligned}
& \mathbf{h}(\mathbf{q})=0 \\
& \mathbf{g}(\mathbf{q}) \leq 0
\end{aligned}
$$

Where $h: \mathfrak{R}^{n} \rightarrow \mathfrak{R}^{z}$ and $g: \mathfrak{R}^{n} \rightarrow \mathfrak{R}^{y}$

The superscripts $z$ and $y$ represent the number of equality and inequality constraints respectively.

The sequential quadratic programming (SQP) algorithm (Nocedal and Wright 2006), one of the widely used algorithms for non-linear constrained optimization, is used to minimize Eq. 2.2. The principal idea is the formulation of a quadratic programming sub-problem based on a quadratic approximation of the Lagrangian function and linearization of the constraints. The QP sub-problem can be written as:

$$
\min _{\delta \mathbf{q}} f\left(\mathbf{q}^{k}\right)+\nabla f^{T}\left(\mathbf{q}^{k}\right) \delta \mathbf{q}+\frac{1}{2} \delta \mathbf{q} \nabla^{2} L\left(\mathbf{q}^{k}\right) \delta \mathbf{q}
$$

Subject to

$$
\begin{aligned}
& \mathbf{h}\left(\mathbf{q}^{k}\right)+\nabla \mathbf{h}\left(\mathbf{q}^{k}\right)^{T} \delta \mathbf{q}=0 \\
& \mathbf{g}\left(\mathbf{q}^{k}\right)+\nabla \mathbf{g}\left(\mathbf{q}^{k}\right)^{T} \delta \mathbf{q} \leq 0
\end{aligned}
$$

$$
L\left(\mathbf{q}, \lambda_{L}, \lambda_{K}\right)=f(\mathbf{q})+\lambda_{L}^{T} \mathbf{h}(\mathbf{q})+\lambda_{K}^{T} \mathbf{g}(\mathbf{q})
$$

The vectors $\lambda_{L}$ and $\lambda_{K}$ in the Lagrangian function represent the Lagrange multipliers corresponding to the equality constraints and the Karush-Kuhan-Tucker multipliers corresponding to the inequality constraints. The QP sub-problem is solved at each major iteration $k$ of the SQP.

For our application, we assume that the constraints are linear and they have the following forms: 


$$
\begin{aligned}
& \mathbf{h}(\mathbf{q})=\mathbf{A q}+\mathbf{b} \\
& \mathbf{g}(\mathbf{q})=\mathbf{C q}+\mathbf{d}
\end{aligned}
$$

By combining Eqs. 2.3 and 2.4, the QP sub-problem at $k^{\text {th }}$ iteration can now be formulated as follows,

$$
\min _{\boldsymbol{\delta} \mathbf{q}}\left\|\mathbf{e}\left(\mathbf{q}^{k}\right)-\mathbf{S}\left(\mathbf{q}^{k}\right) \delta \mathbf{q}\right\|_{2}^{2}+\|\beta \boldsymbol{\delta} \mathbf{q}\|_{2}^{2}
$$

Subject to

$$
\begin{aligned}
& \mathbf{A} \delta \mathbf{q}+\mathbf{h}\left(\mathbf{q}^{k}\right)=0 \\
& \mathbf{C} \delta \mathbf{q}+\mathbf{g}\left(\mathbf{q}^{k}\right) \leq 0
\end{aligned}
$$

In Eq. $2.5 \delta \mathbf{q}$ represents a perturbation in rate and $\mathbf{S}(\mathbf{q})$ is the sensitivity matrix. A single entry of the sensitivity matrix $S_{i j}$ quantifies the changes in arrival time at producer $i$ because of small changes in the rate of well $j$. It is given by

$$
S_{i j}=\frac{\partial t_{i, m}\left(\mathbf{q}^{k}\right)}{\partial q_{j}}
$$

The term $\|\boldsymbol{\beta} \boldsymbol{\delta} \mathbf{q}\|_{2}^{2}$ in Eq. 5 is a regularization term to ensure that the Hessian of the Lagrangian is positive definite (Nocedal and Wright 2006). The Hessian of Eq. 2.5 is given by

$$
\mathbf{H}^{k}=\mathbf{S}^{T}\left(\mathbf{q}^{k}\right) \mathbf{S}\left(\mathbf{q}^{k}\right)+\beta^{2} \mathbf{I}
$$

The weights $\beta$ determine the relative strengths assigned to the rates prior to optimization. In general, the optimization results will be sensitive to the choice of $\beta$ (McLaughlin and Townley 1996).

When the QP sub-problem is subject to no constraints, an LSQR algorithm (Paige and Saunders 1982) can be used to solve the following system of linear equations: 


$$
\left[\begin{array}{c}
\mathbf{S}\left(\mathbf{q}^{k}\right) \\
\beta \mathbf{I}
\end{array}\right][\delta \mathbf{q}]=\left[\begin{array}{c}
\mathbf{e}\left(\mathbf{q}^{k}\right) \\
0
\end{array}\right]
$$

If the QP sub-problem is subject only to equality constrains, the solution can be obtained by solving the following system of linear equations:

$$
\left[\begin{array}{cc}
\mathbf{H}^{k} & \mathbf{A}^{T} \\
\mathbf{A} & 0
\end{array}\right]\left[\begin{array}{l}
\delta \mathbf{q} \\
\boldsymbol{\lambda}_{L}
\end{array}\right]=\left[\begin{array}{c}
\mathbf{S}^{T}\left(\mathbf{q}^{k}\right) \mathbf{e}\left(\mathbf{q}^{k}\right) \\
-\mathbf{h}\left(\mathbf{q}^{k}\right)
\end{array}\right]
$$

If the QP includes inequality constraints, the typical solution involves the active set strategy, through which a sequence of equality constrained problems is solved (Nocedal and Wright 2006).

\subsubsection{Arrival Time and Residual Computation}

We define the arrival time to a producer as the time required for the waterfront to reach the producer from its current position. For example, if the time required by the waterfront to travel from the injector to the producer is 1000 days and the front already has traveled for 600 days, the arrival time is 400 days. For calculation purposes, we compute the arrival time to a producer as the average of the arrival times associated with a set of fast streamlines as defined by the user. In our application, we take the top $20 \%$ of the streamlines.

$$
t_{i, m}^{k}=\left(\frac{1}{N_{f s l, i}} \sum_{l=1}^{N_{f s l, i}} \tau_{l, i} /\left[\frac{d f_{w}}{d S_{w}}\right]_{S_{w}=S_{w f, l}}\right)^{k}
$$

In the above expression, $N_{f s l, i}$ represents the number of the fastest streamlines connected to the producer $i$ belonging to group $m$. As mentioned, we choose a set of the fastest streamlines which consists a portion of $N_{s l, i}$, where $N_{s l, i}$ is the total number of streamlines connected to the producer $i$. The variable $\tau$ represents the usual streamline time-of-flight defined as, 


$$
\tau=\int_{\Sigma} s(\mathbf{x}) d x
$$

where the integral is along the streamline trajectory, $\sum$ and $s(\mathrm{x})$ is the 'slowness' defined as the reciprocal of the total interstitial velocity

$$
s(\mathbf{x})=\frac{1}{|v(\mathbf{x})|}=\frac{A(\mathbf{x}) \phi(\mathbf{x})}{q_{s l}}
$$

The variables $q_{s l}, A(\mathbf{x})$ and $\phi(\mathbf{x})$ represent the flow rate, streamtube area, and porosity along individual streamlines. Note that Eq. 2.10 acknowledges that the travel time of the waterfront is related to the streamline time of flight through the fractional flow relationship.

So far we have discussed the calculation of the arrival time for individual producers. The next step is to compute the desired arrival time in Eq. 2.1. This should be the same for all producers within group $m$. The desired arrival time, $t_{d, m}^{k}$ for group $m$ at iteration $k$ is chosen so as to minimize the variance as follows:

$$
\min _{t_{d, m}^{k}} \frac{1}{N_{p r o d, m}} \sum_{i=1}^{N_{p r o d, m}}\left(t_{d, m}^{k}-t_{i, m}\left(\mathbf{q}^{k}\right)\right)^{2}
$$

which results in the following,

$$
t_{d, m}^{k}=\frac{\sum_{i}^{N_{\text {prod }, m}} t_{i, m}\left(\mathbf{q}^{k}\right)}{N_{\text {prod }, m}}
$$

Note that $t_{d, m}$ is recomputed at each iteration of the optimization to ensure minimization of Eq. 2.5. Finally, the residuals for each producer are computed using Eq. 2.1b. 


\subsubsection{Analytical Sensitivity Calculation}

The next step is to compute the coefficients of the sensitivity matrix $S_{i j}$ analytically. By Combining Eqs. 2.6 and 2.10, we can write $S_{i j}$ as follows,

$$
S_{i j}=\frac{1}{N_{f s l, i}} \sum_{l=1}^{N_{f s l, i}} \frac{\partial \tau_{l, i}}{\partial q_{j}} /\left[\frac{d f_{w}}{d S_{w}}\right]_{S_{w}=S_{w f, l}}
$$

Using the chain rule, the partial derivative in Eq. 2.15 can be written as

$$
\frac{\partial \tau_{l, i}}{\partial q_{j}}=\frac{\partial \tau_{l, i}}{\partial q_{s l, i}} \frac{\partial q_{s l, i}}{\partial q_{j}}
$$

The expression $\partial \tau_{l, i} / \partial q_{s l, i}$ represents the change in time of flight along individual streamlines connected to producer $i$ because of changes in the total flow rate along the streamline. If we assume that the streamlines do not shift because of small perturbations in wells rate, then this partial derivative can be computed analytically using Eq. 2.11 and Eq. 2.12.

$$
\frac{\partial \tau_{l, i}}{\partial q_{s l, i}}=\int_{\Sigma} \frac{\partial s(\mathbf{x})}{\partial q_{s l, i}} d x=-\int_{\Sigma} \frac{s(\mathbf{x})}{q_{s l, i}} d x=-\frac{\tau_{l, i}}{q_{s l, i}}
$$

The second partial derivative in Eq. 2.16, $\left(\partial q_{s l, i} / \partial q_{j}\right)$ represent the change in the total production rate along a streamline connected to producer $i$ because of a change in the total rate of well $j$. Recall that well $j$ can be either a producer or an injector. Let's consider first the case when it is a producer. In this case, the derivative will vanish for $i$ $\neq j$ because of the assumption that the streamlines do not shift for small perturbations in well rates. If $i=j$, the well rate and the flow rate along individual streamline are related as follows,

$$
q_{i}=N_{s l, i} q_{s l, i}
$$


Using Eqs. 17 and 18, we can now rewrite Eq. 2.16 as

$$
\begin{aligned}
& \frac{\partial \tau_{s l, i}}{\partial q_{j}}=-\frac{\tau_{s l, i}}{q_{j}} \quad \forall i=j \\
& \frac{\partial \tau_{s, i}}{\partial q_{j}}=0 \quad \forall i \neq j
\end{aligned}
$$

After substituting Eq. 2.19 in Eq. 2.15, we have the analytical form of the sensitivities with respect to production rates.

$$
\begin{aligned}
& S_{i j}=-\frac{t_{i, m}}{q_{j}} \quad \forall i=j \\
& S_{i j}=0 \quad \forall i \neq j \\
& j \text { is a producer }
\end{aligned}
$$

In Eq. 2.20, we assume that $t_{i, m}$ is sensitive only to the production of producer $i$. The sensitivity of $t_{i, m}$ is negligible with respect to other producers.

Similar assumptions hold when computing the sensitivity with respect to injection rates. Following a similar approach, the analytical sensitivity with respect to the injection rate can be written as follows,

$$
\begin{aligned}
& S_{i j}=-\frac{\sum_{l=1}^{N_{f s l, i, j}} \tau_{l, i, j} /\left[\frac{d f_{w}}{d S_{w}}\right]_{S_{w}=S_{w f, l}}}{q_{j} N_{f s l, i}} \text { if } N_{f s l, i, j} \neq 0 \\
& S_{i j}=0 \text { if } N_{f s l, i, j}=0 \\
& j \text { is an injector }
\end{aligned}
$$

The variable $N_{f s l, i, j}$ is the number of the fastest streamlines connecting a producer $i$ to an injector $j$. This number represents only a portion of $N_{f s l, i}$, the total number of the fastest streamlines connected to the producer $i$. If the injector $j$ is not connected to producer $i$ through a fast streamline i.e. $\left(N_{f s l, i, j}=0\right)$, then the arrival time at producer $i$ is not sensitive 
to a perturbation in the rate of injector $j$. This assumption will be examined later in the discussion section.

It is worthwhile to emphasize here that the computation of the sensitivity matrix discussed here require a single flow simulation using either a streamline or a finitedifference model. Use of finite-difference model will require the additional calculations associated with the streamlines and time of flight. In contrast, if we compute the

sensitivity matrix by numerical perturbation, it will require $1+N_{p r o d}+N_{i n j}$ simulation runs if both production/injection rates were optimized. Thus, analytical computation of sensitivity matrix can lead to a substantial savings in computation time.

\subsection{Application and Discussion}

In this section, we first use the 2D homogenous case discussed before to examine the validity of the assumptions made for the analytical sensitivities in Eqs. 2.20 and 2.21 and their impact on the convergence of the solution. We then examine the impact of mobility ratios on the rate optimization process. Next, we illustrate our proposed approach using an application to smart wells with inflow control valves (ICV). Finally, the feasibility of our approach is demonstrated through a field example.

\subsubsection{Sensitivity Comparisons and Convergence Behavior: 2D Homogeneous Example}

We first examine with the validity of the assumptions in the analytical sensitivity computations (Eqs. 2.20 and 2.21) by comparison with the numerical perturbation method. We have assumed that the streamlines do not shift significantly because of small local perturbations in rates. Also, the waterfront arrival time at producer $i$ will be mainly sensitive to production at producer $i$ and relatively insensitive to others. Table 2.2 shows the sensitivity of the arrival time at the nine producers with respect to their production rates using the numerical perturbation method. 
Table 2.2 Sensitivity of arrival time at producers with respect to production rates

\begin{tabular}{|l|l|l|l|l|l|l|l|l|l|}
\hline$S_{i j}$ & $j=1$ & $j=2$ & $j=3$ & $j=4$ & $j=5$ & $j=6$ & $j=7$ & $j=8$ & $j=9$ \\
\hline$i=1$ & -1.7098 & -0.0147 & 0.0242 & -0.0179 & 0.0411 & 0.0334 & 0.0225 & 0.0321 & 0.0283 \\
\hline$i=2$ & -0.1193 & -2.7960 & -0.1155 & 0.0378 & -0.0177 & 0.0460 & 0.0136 & 0.0152 & 0.0088 \\
\hline$i=3$ & 0.0198 & -0.0149 & -1.7095 & 0.0256 & 0.0389 & -0.0135 & 0.0258 & 0.0302 & 0.0160 \\
\hline$i=4$ & -0.1201 & 0.0455 & 0.0184 & -2.8039 & -0.0180 & 0.0193 & -0.1228 & 0.0428 & 0.0076 \\
\hline$i=5$ & -0.4493 & -0.3045 & -0.4479 & -0.3092 & -3.5780 & -0.3014 & -0.4511 & -0.3050 & -0.4584 \\
\hline$i=6$ & 0.0136 & 0.0418 & -0.1179 & 0.0088 & -0.0194 & -2.7978 & 0.0125 & 0.0401 & -0.1255 \\
\hline$i=7$ & 0.0184 & 0.0340 & 0.0318 & -0.0227 & 0.0384 & 0.0345 & -1.7170 & -0.0171 & 0.0139 \\
\hline$i=8$ & 0.0136 & 0.0178 & 0.0180 & 0.0368 & -0.0185 & 0.0456 & -0.1232 & -2.7990 & -0.1265 \\
\hline$i=9$ & 0.0302 & 0.0310 & 0.0227 & 0.0299 & 0.0402 & -0.0170 & 0.0221 & -0.0167 & -1.7124 \\
\hline
\end{tabular}


The shaded values represent the sensitivity of the arrival time of a specific producer to its production rate. Clearly, these elements are one to two orders of magnitude higher than the off-diagonal elements which have been neglected in our analysis. Similarly for the injection wells we assume in Eq. 2.20 that if there is no connection between the injector $j$ and the producer $i$, then the sensitivity of the arrival time at the producer $i$ is negligible with respect to injector $j$. To illustrate this, Figure 2.12 shows the streamline patterns for the $2 \mathrm{D}$ homogenous example at the first iteration during optimization. Injector I1 is connected only to four producers: P1, P2, P4, and P5. The sensitivity of the arrival time with respect to injection rates computed using the numerical perturbation method is summarized in Table 2.3. A small perturbation in the injection rate of I1 has a sizable impact only on the arrival times at P1, P2, P3, and P4. Same behavior is observed for the other injectors.

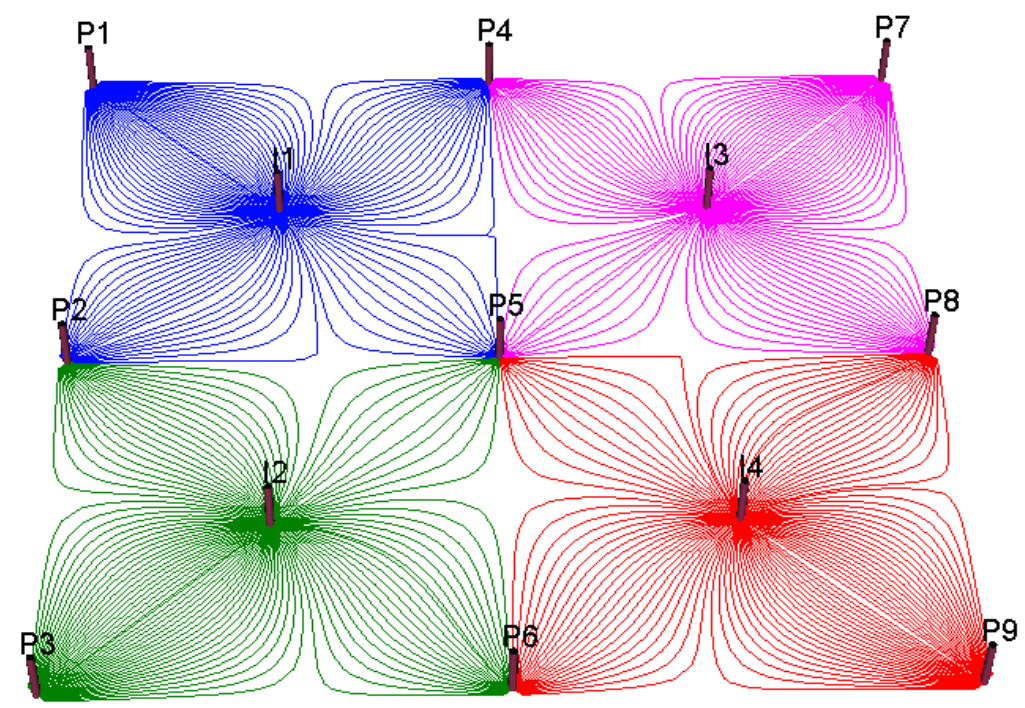

Figure 2.12 Streamlines: 2D homogenous example 
Table 2.3 Sensitivity of arrival time at producers with respect to injection rates

\begin{tabular}{|l|l|l|l|l|}
\hline$S_{i j}$ & $j=1$ & $j=2$ & $j=3$ & $j=4$ \\
\hline$i=1$ & -0.2953 & -0.0297 & -0.0285 & -0.0306 \\
\hline$i=2$ & -0.7248 & -0.7258 & -0.0682 & -0.0719 \\
\hline$i=3$ & -0.0308 & -0.2976 & -0.0295 & -0.0320 \\
\hline$i=4$ & -0.7253 & -0.0715 & -0.7230 & -0.0724 \\
\hline$i=5$ & -1.7683 & -1.7704 & -1.7675 & -1.7706 \\
\hline$i=6$ & -0.0703 & -0.7260 & -0.0693 & -0.7269 \\
\hline$i=7$ & -0.0316 & -0.0324 & -0.2951 & -0.0328 \\
\hline$i=8$ & -0.0705 & -0.0718 & -0.7232 & -0.7279 \\
\hline$i=9$ & -0.0299 & -0.0299 & -0.0291 & -0.2963 \\
\hline
\end{tabular}

Next, we briefly examine the convergence characteristics of the minimization using the analytical sensitivities. As expected, an accurate calculation of the sensitivities using the numerical perturbation generally leads to faster convergence and less iterations as shown in Figure 2.13a. For the 2D homogeneous, it requires 5 iterations to minimize the norm of the residuals to 0.02 . In contrast, the analytical sensitivities require 8 iterations to minimize the norm of the residuals to 0.09 . However, if we compare the associated costs, the numerical method requires 71 simulation run (13 simulation run / iteration) whereas the analytical method requires only 8 (one simulation run / iteration) as shown in Figure 2.13b. This clearly demonstrates the potential computational advantage of our method for large-scale field applications. 


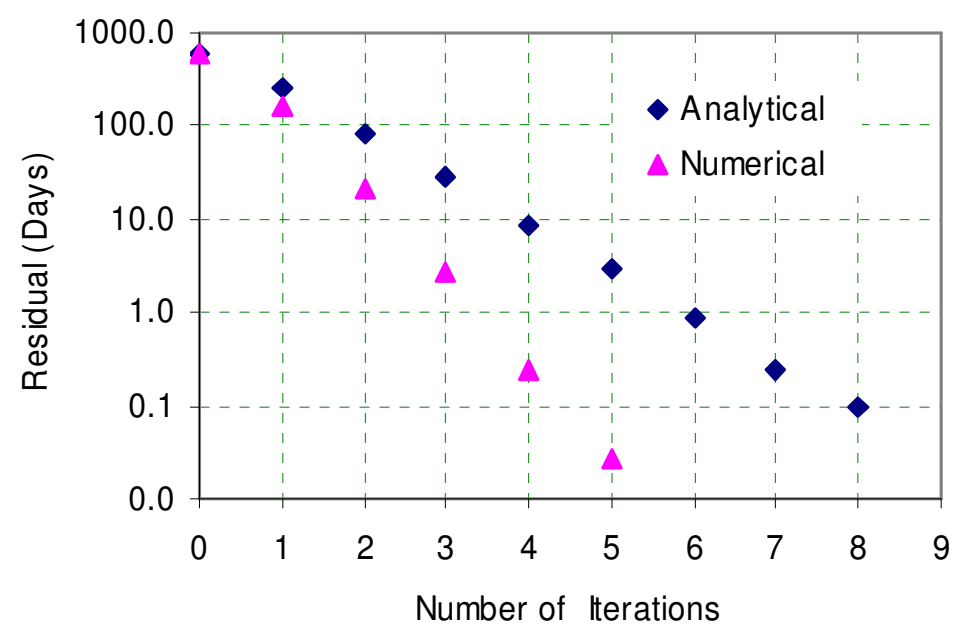

a. Comparison in terms of numbers of iterations

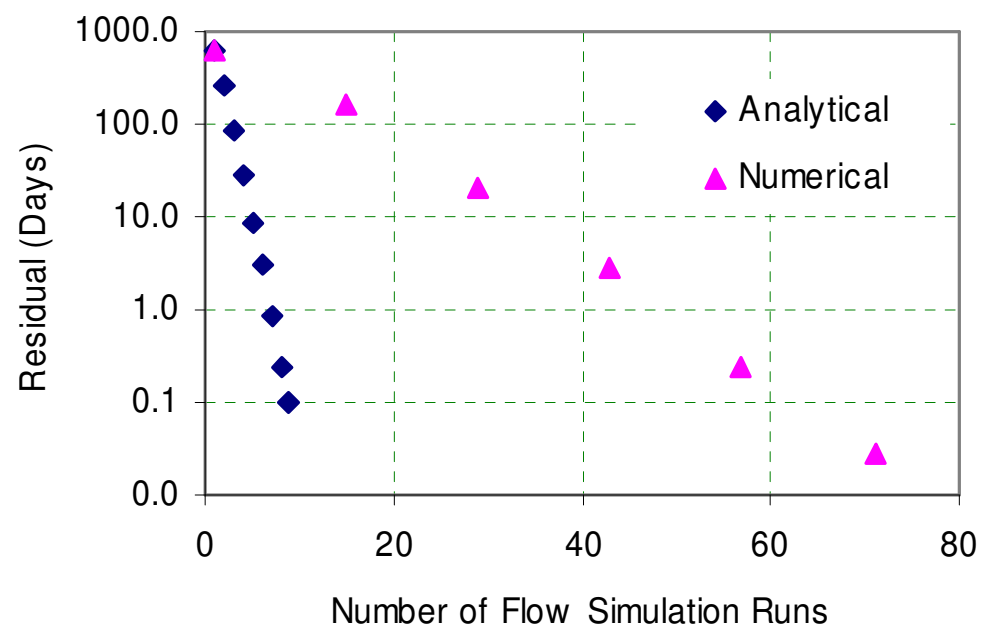

b. Comparison in terms of numbers of simulation runs

Figure 2.13 Comparison between analytical and numerical sensitivities 


\subsubsection{Impact of Mobility Ratio: 2D Heterogeneous Example}

We have seen that the optimal rates do not change with time significantly in Figure 2.10a and $\mathbf{b}$ when $M=1$. We now examine the impact of changing $M$ on the optimization process. We will use the same 2D heterogeneous example described in Figure2.7 for two different mobility ratio displacements, $\mathrm{M}=0.25$ and 10 .

For favorable mobility ratio $(M=0.25)$, Osako et al. (2004) showed that the transverse flux associated with viscous cross flow is generally quite significant. This requires updating the pressure field more frequently. We performed the optimization at multiple time intervals of equal size viz. 100 Days. At each time interval, the pressure field is updated, the streamline and time of flight are recomputed. The optimization is then carried out using the new sensitivities. Figure 2.14a and b show the optimization results for $M=0.25$. A higher variability in well rates can be observed here compared to the unit mobility ratio case in Figure 2.10a and b. The optimized water arrival when $\mathrm{M}=0.25$ is around 950 Days. For the unfavorable mobility ratio case $(M=10)$, the cross flow is not significant and we require fewer pressure updates. ${ }^{16}$ Figures $\mathbf{2 . 1 5 a}$ and $\mathbf{b}$ show that the rates are almost constant throughout the optimization process. The optimized water arrival occurred at 350 Days. 


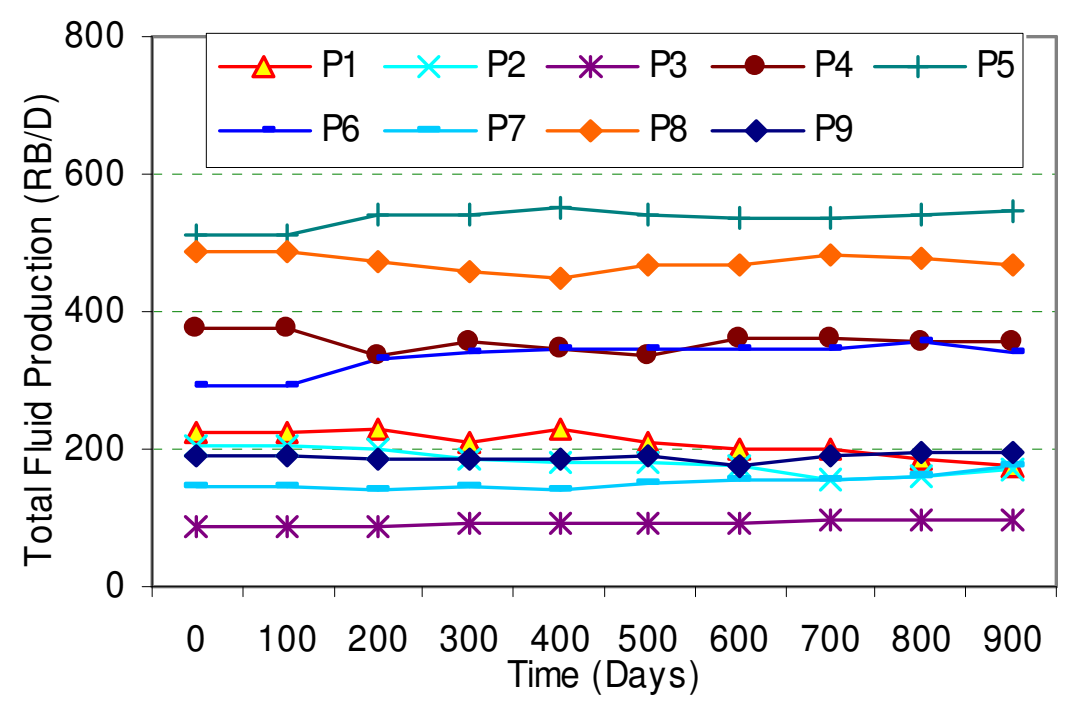

a. Total Fluid Rate

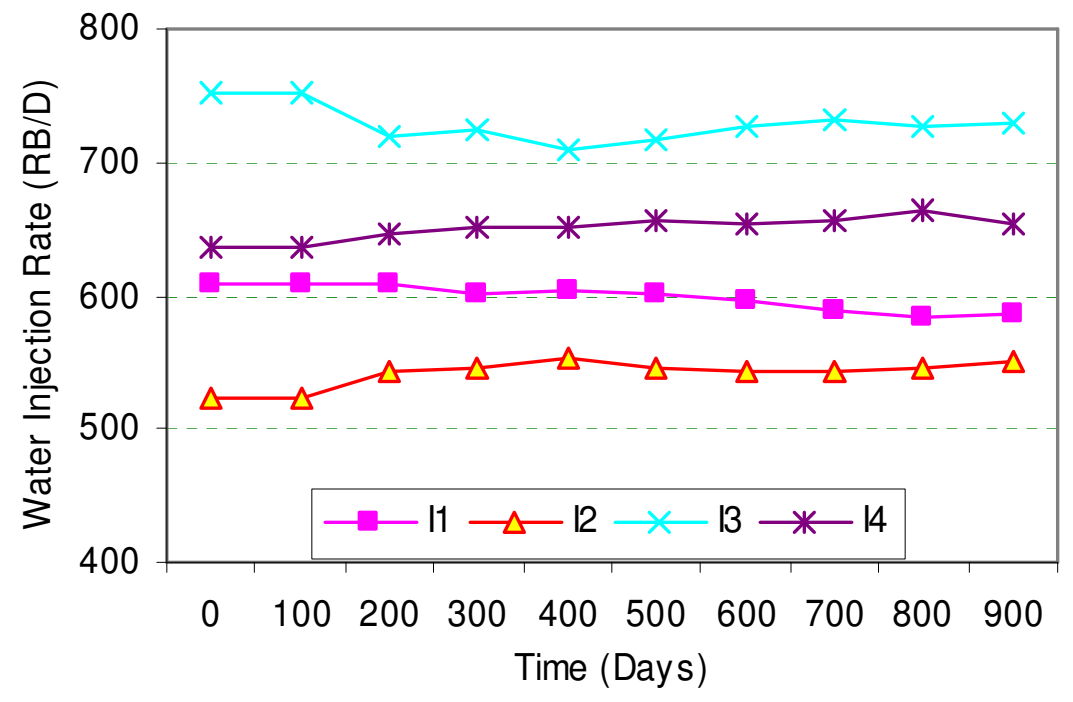

b. Water Injection Rate

Figure 2.14 Production/injection rates after rate optimization, $M=0.25: 2 D$ heterogeneous example 


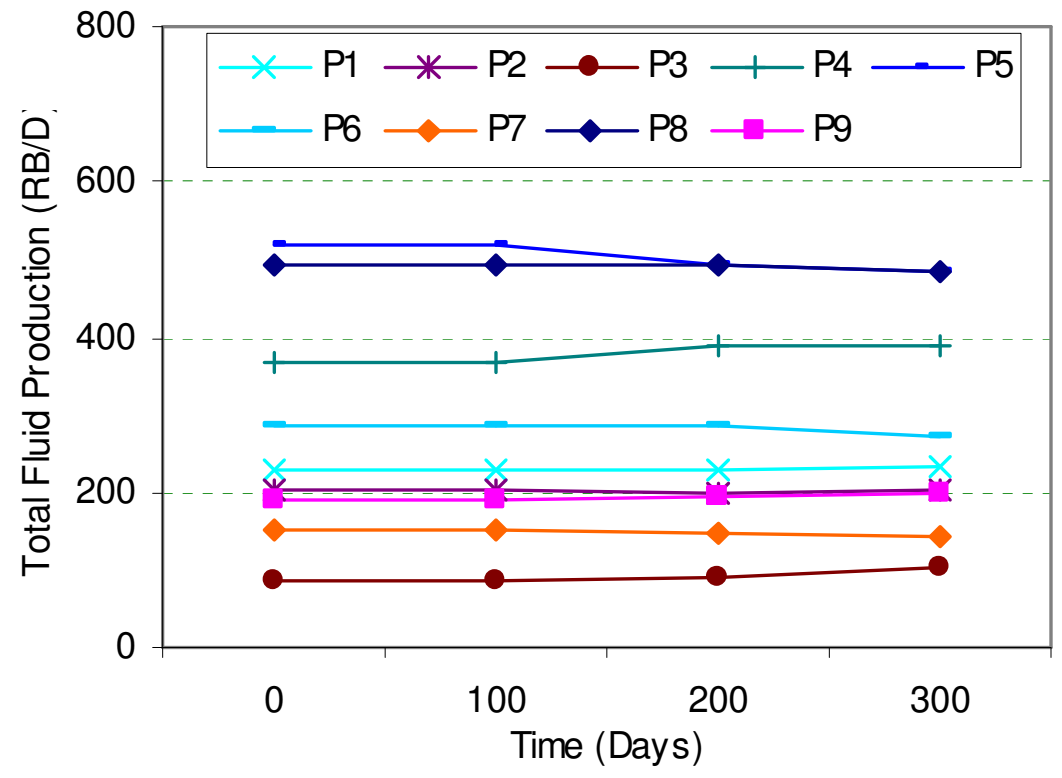

a. Total fluid rate

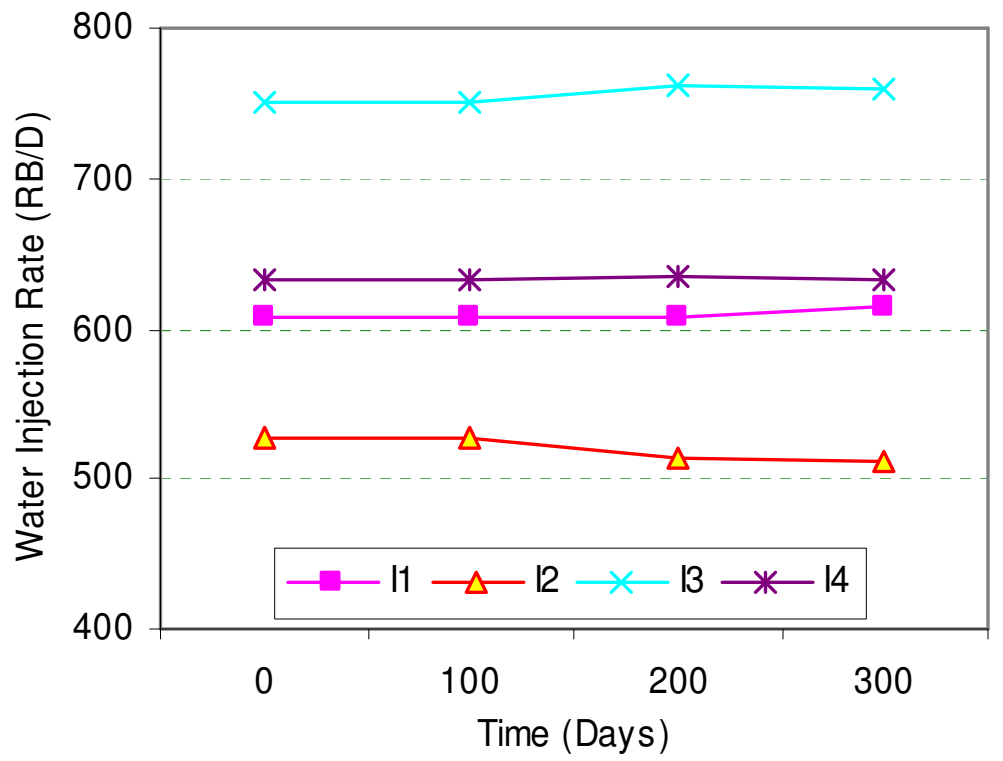

b. Water injection rate

Figure 2.15 Production/injection rates after rate optimization, $M=10$ : 2D heterogeneous example 
To summarize the effects of mobility ratio, for favorable mobility ratios we need to perform the optimization at multiple time intervals to account for pressure updates. The selection of the time step size depends, among others, on transverse fluxes arising from mobility and unsteady state effects according to guidelines provided by Osako et al. (2004).

\subsubsection{A Smart Well Example}

This example demonstrates the applicability of our approach to mange waterfront using smart wells with inflow control valves (ICV). The example follows closely from that of Brouwer et al. (2001) where the goal is to counteract the adverse impact of a high permeability streak causing premature water breakthrough during waterflooding.

The example consists of a two dimensional reservoir modeled with 50x50x1 gridblocks. The dimensions of each gridblock are, $\Delta x=\Delta y=\Delta z=33 \mathrm{ft}$, and the porosity is assumed to be constant and equals to 0.225 . The reservoir has a permeability of $100 \mathrm{md}$ except in the middle where there is a permeability streak of $400 \mathrm{md}$. The width of this streak is 10 gridbocks and it extends throughout the reservoir from east to west. The wells are placed such that the flow direction is parallel to the high permeability streak as shown in Figure 2.16. There are two wells: 1 producer and 1 injector. Both wells are completed as smart-horizontal wells with 50 controllable segments (ICVs) for each well. Each griblock along the well path represents a segment and will be treated as an individual well for flow simulation purposes. Thus, we will consider optimizing 100 wells: 50 producers and 50 injectors. As a base case, we assume that each segment has an equal rate of $100 \mathrm{RB} / \mathrm{D}$ for both wells. Obviously, the segments crossing the high permeability streak will result in an early water arrival as shown in Figure 2.17a. The

water reaches the producer in 350 days while the recovery is $48 \%$ of OIIP. Our aim is to improve the sweep efficiency though optimizing the rates of the individual segments to equalize the water front arrival times. The results after optimization are shown in Figure 2.17b. We have been able to delay the water arrival by about 200 days and reach to 
almost an even water front at arrival time. The recovery at breakthrough is improved by $32 \%$, a very significant increase.

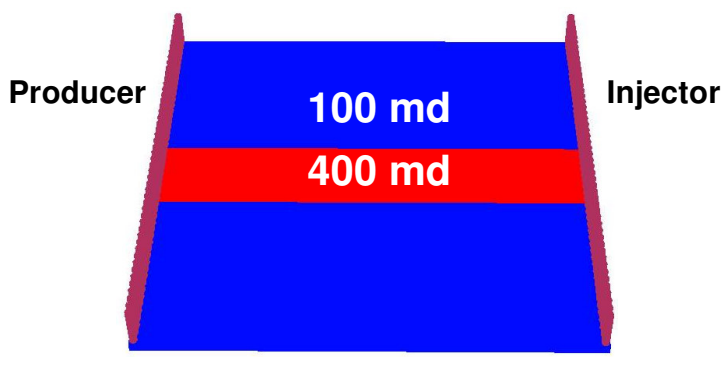

Figure 2.16 2D example for smart wells optimization

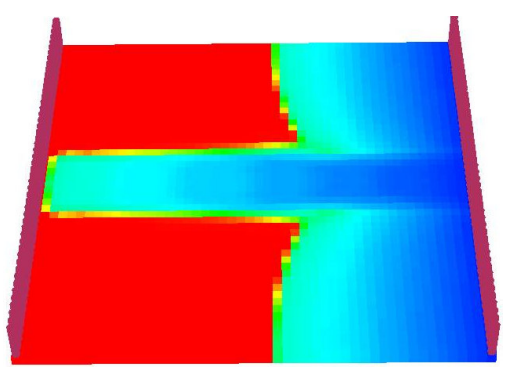

a. Prior to rate optimization, water arrival at 350 days, recovery $=\mathbf{4 8 \%}$

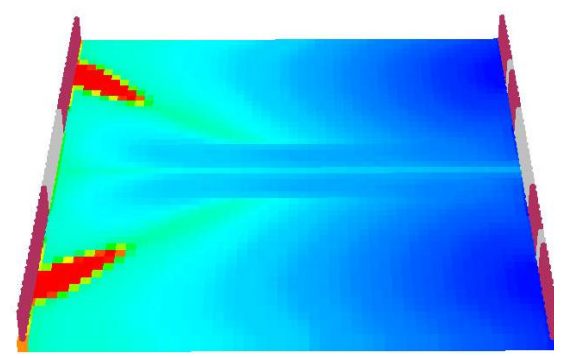

b. After rate optimization, water arrival at 575 days, recovery $=\mathbf{8 0 \%}$

Figure 2.17 Oil saturation at arrival time: 2D smart well example 
The water cut history for each segment before and after the optimization is presented in Figure 2.18a and b. Clearly, the rate optimization effectively diminishes the impact of the high permeability streak. After optimization all segments water out almost at the same time. The optimization was performed for one time interval as we assumed $M=1$. Throughout the optimization process the total production rate is kept constant and is equal to the field injection rate. The upper bound for injection/production for each segment is set at $500 \mathrm{RB} / \mathrm{D}$, and the lower bound is zero RB/D. The optimized injection/production rates for each segment are presented in Figure 2.19a and b. The results are quite intuitive. The algorithm suggests shutting in the segments that lie primarily on the high permeability streak and assigning higher rates for segments located towards the toe or the tail of the horizontal section. Allocating rates in such manner helps equalize the time of flight for the streamlines and leads to almost an equalized front arrival at the producer. Similar observations were reported by Brouwer et al. (2001).

\subsubsection{A Field Example}

We have applied our approach to a $\mathrm{CO}_{2}$ pilot project area in the Goldsmith San Andres Unit (GSAU), a dolomite formation located in west Texas. The pilot area covers around 320 acres with an average thickness of 100ft. It consists of nine inverted 5-spot patterns and has over 50 years of production history before the initiation of the $\mathrm{CO}_{2}$ pilot project in 1996. Extra wells located outside the pilot area were included in this study to account for correct boundary conditions. The extended study area was descretized into $58 \times 53 \times 10$ mesh or a total of 30,740 grid cells containing 11 water injectors and 31 producers. The porosity field, shown in Figure 2.20a, is obtained by a Sequential Gaussian Simulation using the well log and seismic data. The permeability field, shown in Figure 2.20b, is generated via a cloud transform based on the porosity-permeability relationship. In this example, we have only two phase flow because the reservoir pressure is kept above the bubble point pressure during the waterflood. 


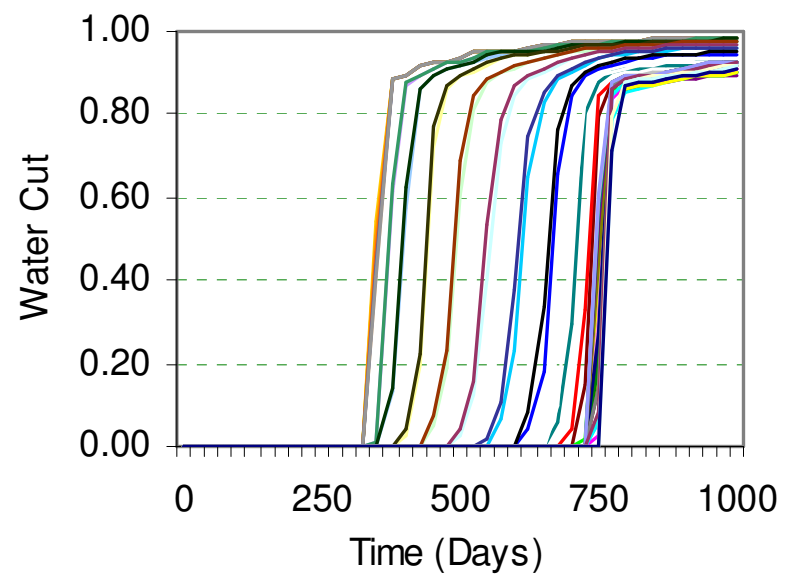

a. Prior to rate optimization

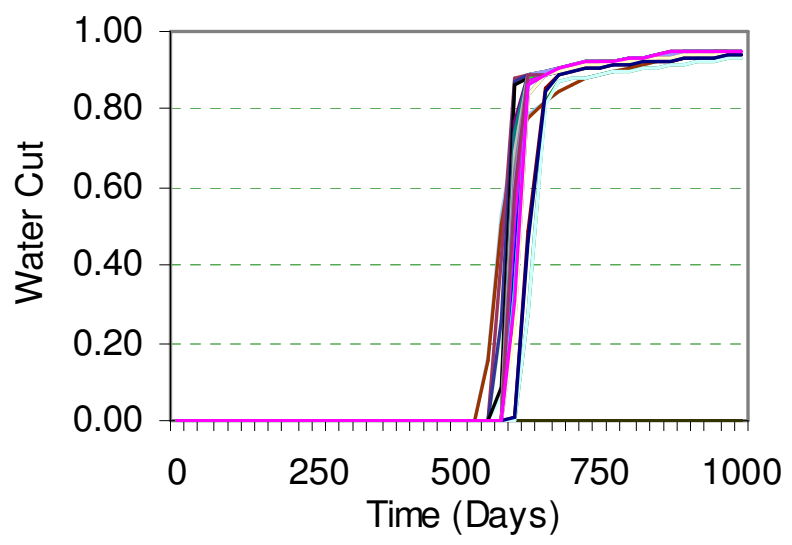

b. After rate optimization

Figure 2.18 Water cut profile for 50 segments along the horizontal section: smart well example 


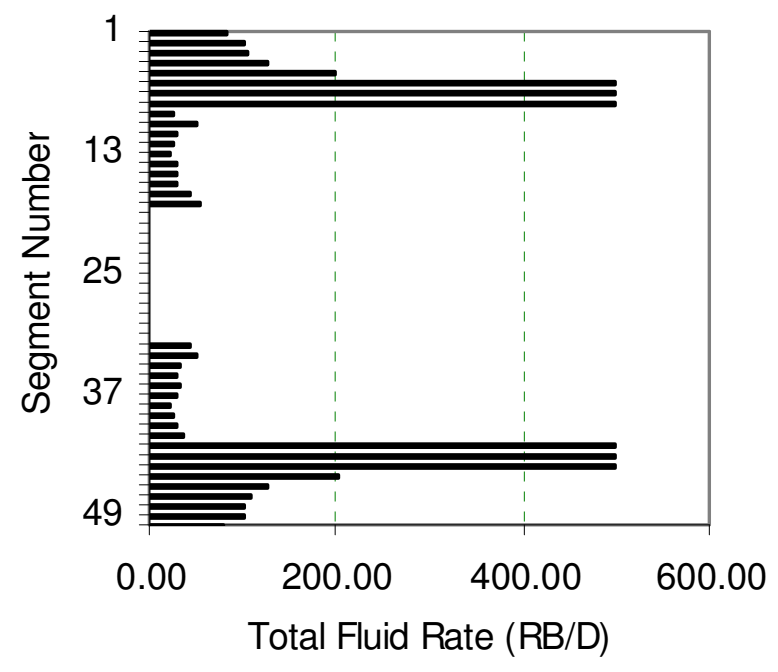

19a. Total fluid production rate

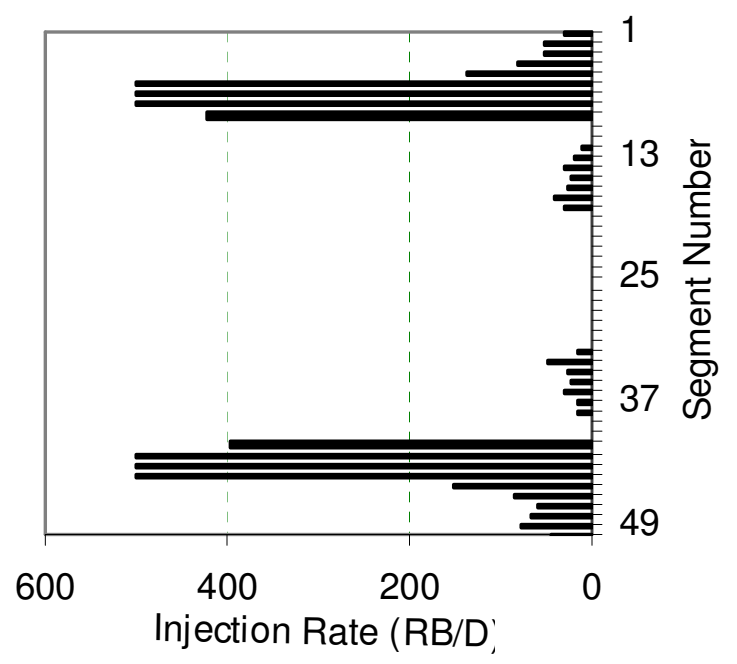

19b. Water injection rate

Figure 2.19 Total fluid production and injection after rate optimization: smart well example 


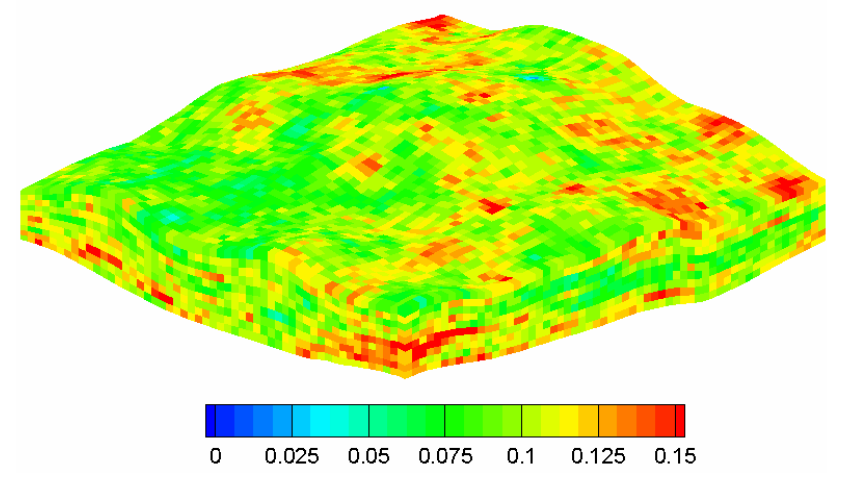

\section{a. Porosity distribution}

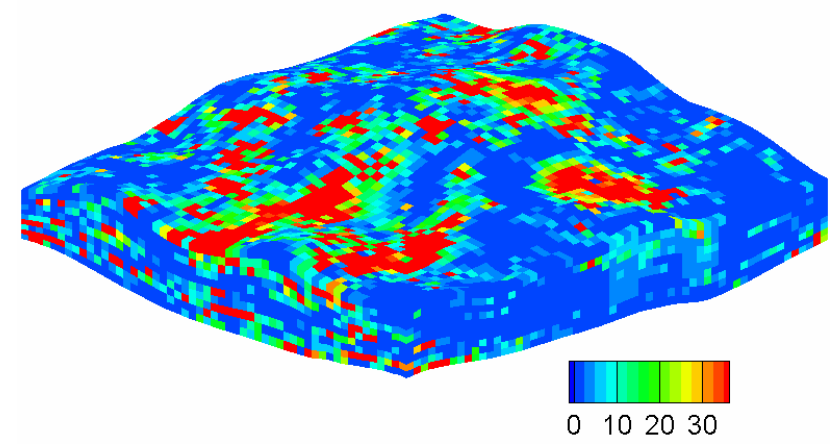

b. Permeability distribution

\section{Figure 2.20 Porosity and permeability distribution: field example}

We used the actual field production/injection history during the first 14 years as the base case for our optimization. Our goal is to show that our approach will suggest a new production strategy resulting in a better sweep. To account for the changing production rate and different starting times of the injection and production wells, 7 pressure time-steps were used during the first 14 years. At the beginning of each time interval, we start with the base case production strategy and then implement our approach. The optimization was performed only on the production wells, while injection wells were kept the same as the base case controlled by the bottom-hole pressure. The production wells were subdivided into different groups, with each group having a 
desired arrival time defined as the average of arrival times for the wells in the group as discussed in the mathematical formulation section. The well groups are chosen in the following manner:

- Compute the arrival time for each producer as the average time of flight of the fastest streamlines to the producer. For the field case, we took the arrival time as the time required to travel from the injector to the producer. This is unlike the previous cases, where it was computed as the travel time from the waterfront to the producer. This revised definition now allows us to continue the optimization even after water breakthrough.

- Identify the injector that contributes most of the fastest streamlines to each producer.

- Choose the producers with the same contributing injector as one group.

This process is repeated every time-step because of changing production/injection rates and infill drilling. To illustrate the procedure, Figure 2.21a shows three groups of production wells connected to three injectors: I1, I5, and I11. These groups were selected for the first time-step in the first iteration. Because of changing field condition during the second time-step and injector I2 being activated, the groups connected to I1 and I11 were changed as shown in Figure 2.21b. 


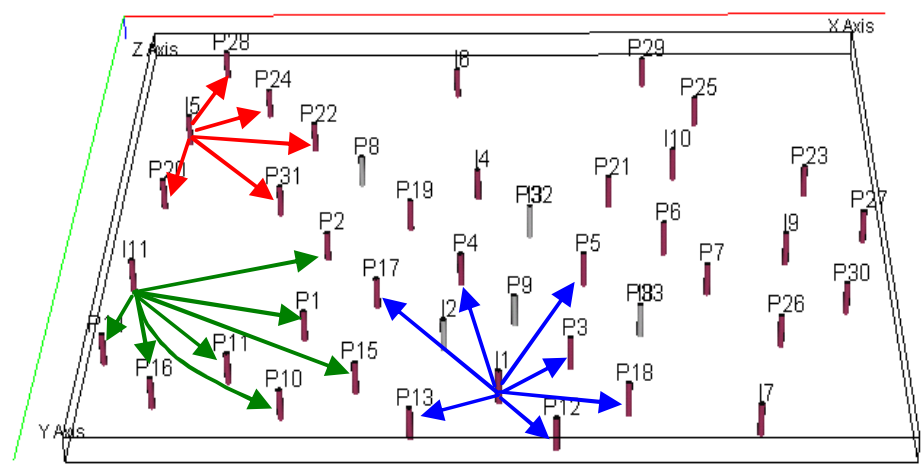

a. Wells groups before activating injector I2: first time-step

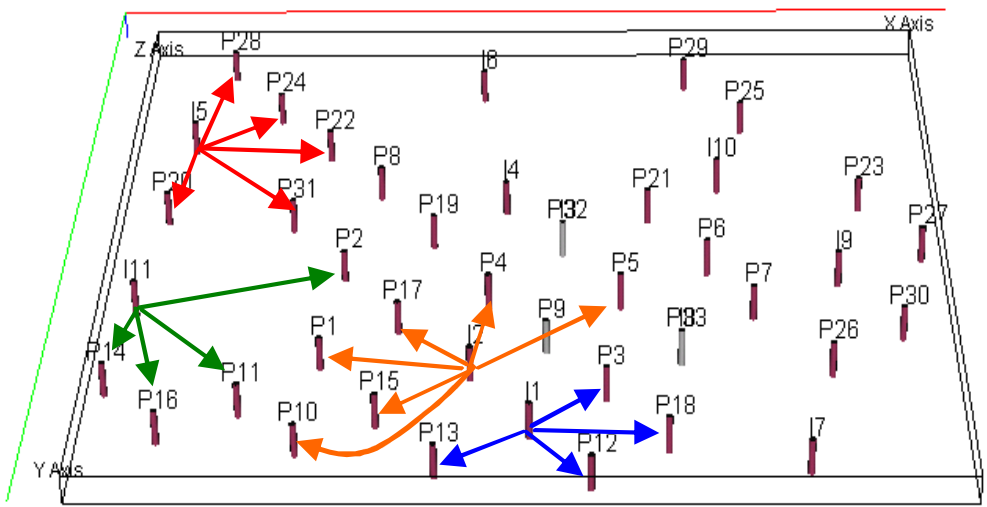

b. Wells groups after activating injector I2: second time-step

Figure 2. 21 Wells groups at the first and second time-steps for the field example. (inactive wells are shown in grey) 
We implemented three operational constraints throughout the optimization process: (i) Field injection and total fluid production to be kept the same as the base case. (ii) Well total production should not to exceed 800 STB/D. (iii) Well flowing bottom-hole pressure should not go below 1000 psi. Those constraints were met during the optimization process as shown in Figures 2.22 and 2.23.

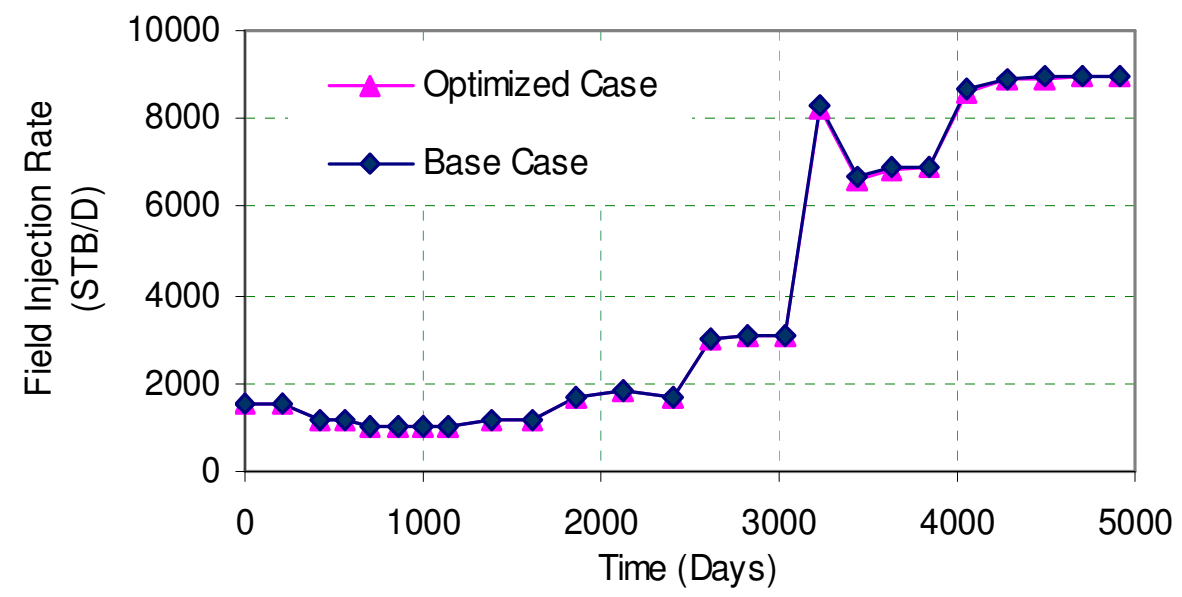

Figure 2.22 Field water injection before and after optimization acknowledging field constraints 


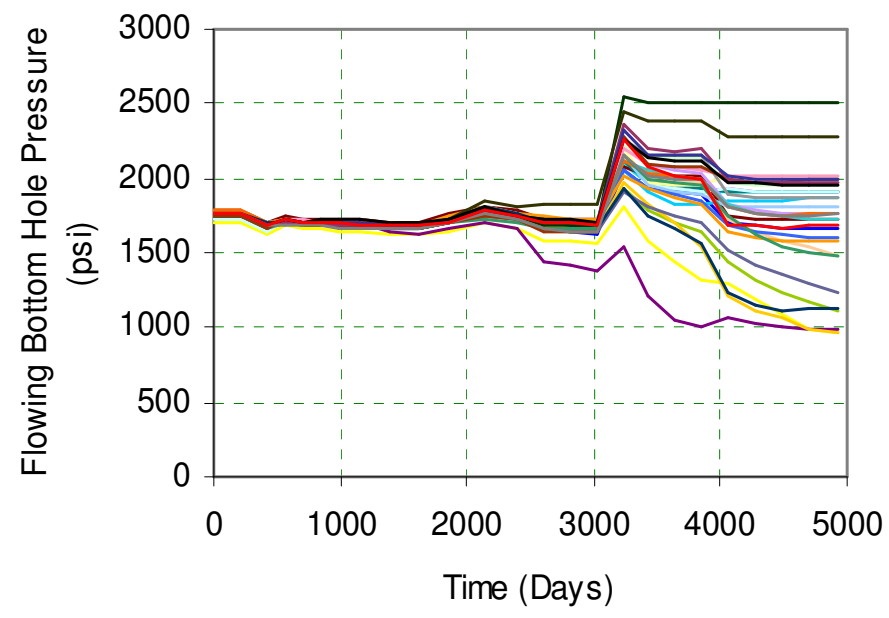

\section{a. Flowing bottom hole pressure}

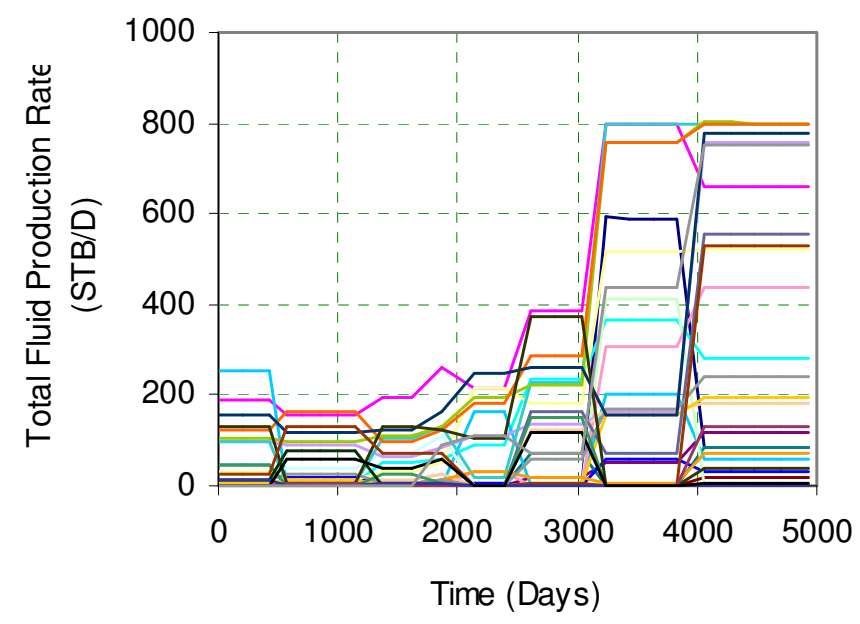

b. Total fluid production

\section{Figure 2.23 Performance of 33 wells after optimization: field example}

The optimization resulted in delaying of the field water breakthrough by almost 3 years and reduced the water cut as shown in Figure 2.24a. More cumulative oil was produced during the period of optimization resulting in a higher ultimate recovery as shown in Figure 2.24b. We can reach the same conclusions if we examine the oil saturation profile before and after the optimization. 


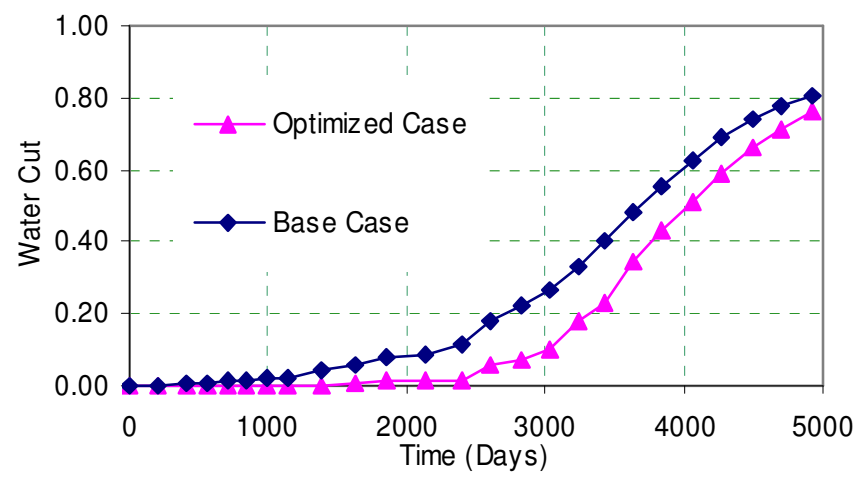

a. Water cut vs. time

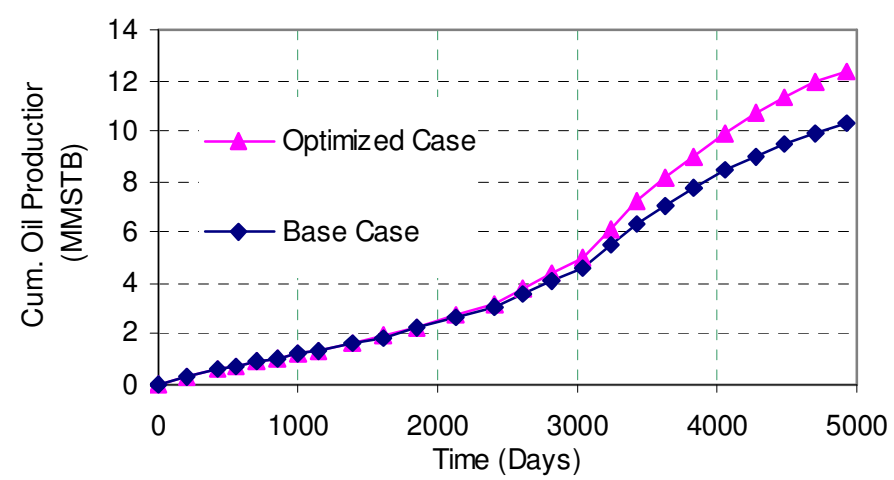

b. Cumulative oil production vs. time

\section{Figure 2.24 Performance plots for the field example}

Difference maps between the base and optimized case in terms of oil saturation are shown in Figure 2.25, indicating the bypassed oil that was recovered because of optimization. Figure $\mathbf{2 . 2 6}$ shows the performance of P28 and 31 before and after optimization. Those wells belong to the same group as shown in Figure 2.21 and they located in an area where there has been a significant improvement in oil recovery as shown in Figure 2.25. The performance shows that the improvement occurred because of reallocating rate in that area. Producer 28 was produced at a lower rate than the base case while P31 was produced at a higher rate as shown in Figure 2.26b. 


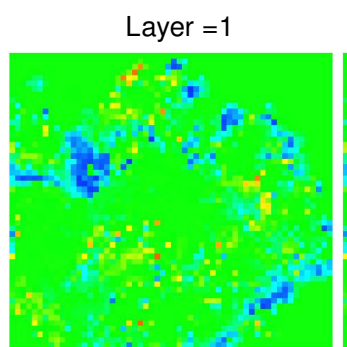

Layer $=6$
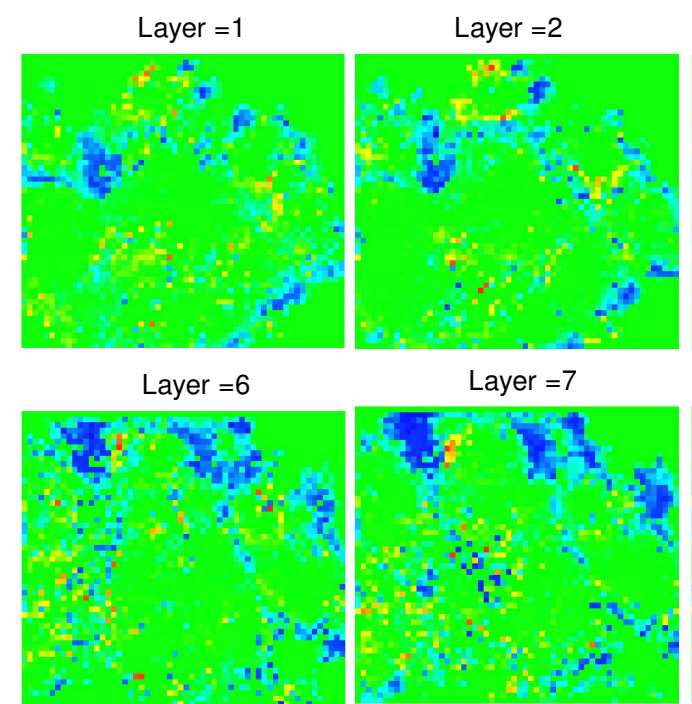

Layer $=7$
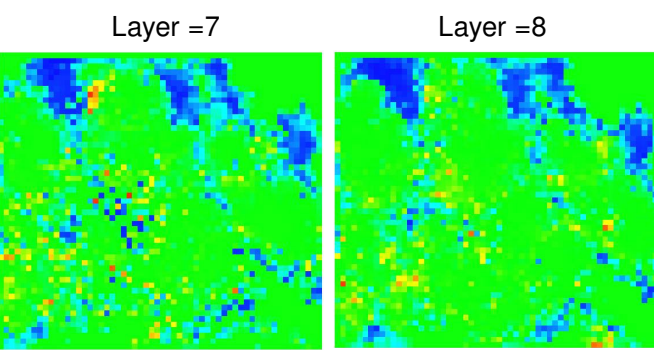

Layer $=3$
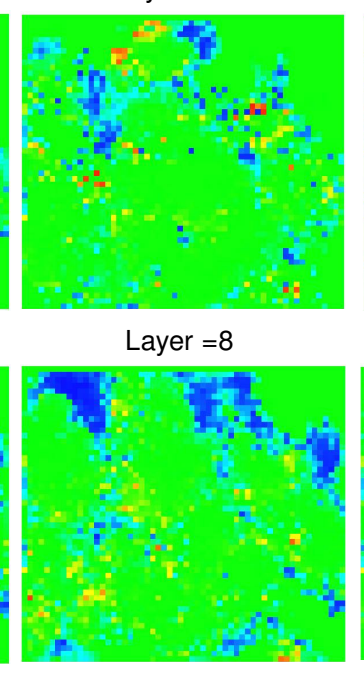

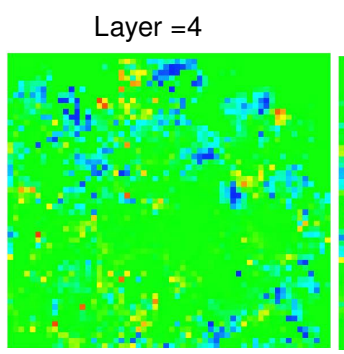

Layer $=9$

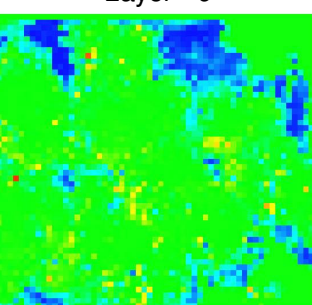

Layer $=5$

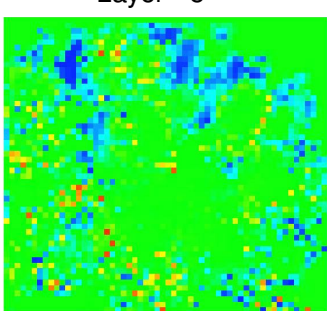

Layer $=10$

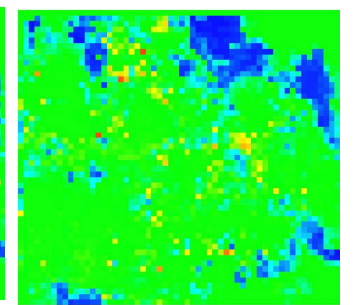

$-0.39650$

$-0.19825$

$-0.0000$

0.19825

0.39650

Figure 2.25 Difference maps in terms of oil saturation between the base case and the optimized case (So, base - So, optimized) @ 5000 days: field example 

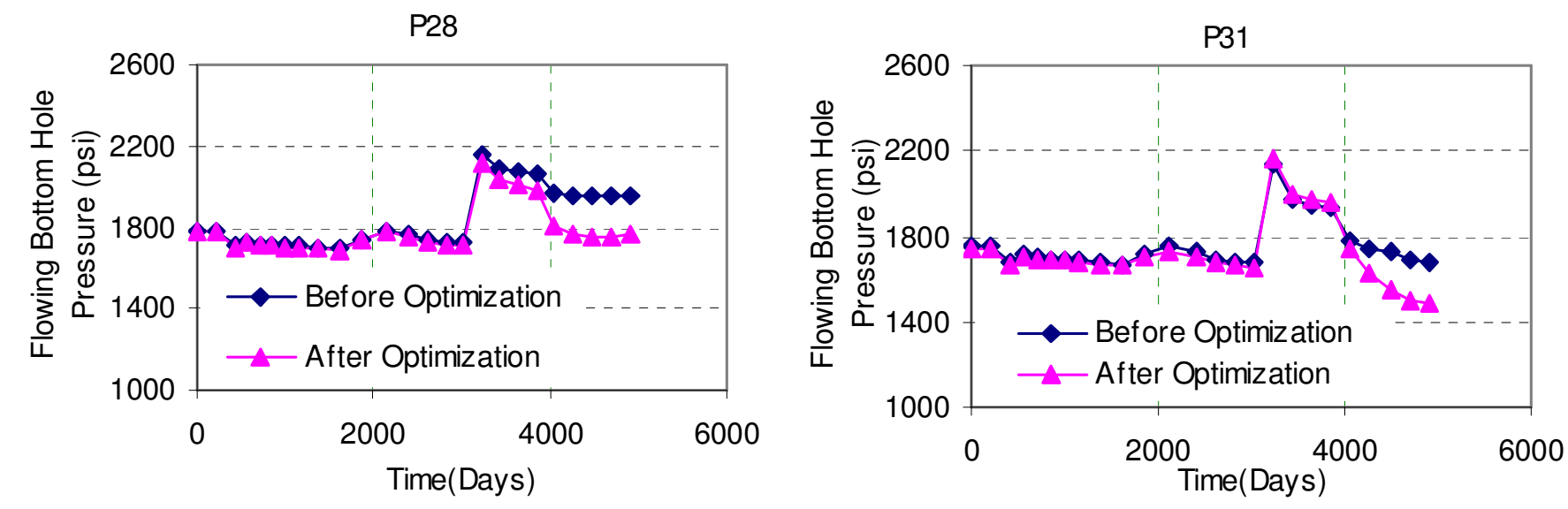

a. Flowing bottom hole pressure vs. time
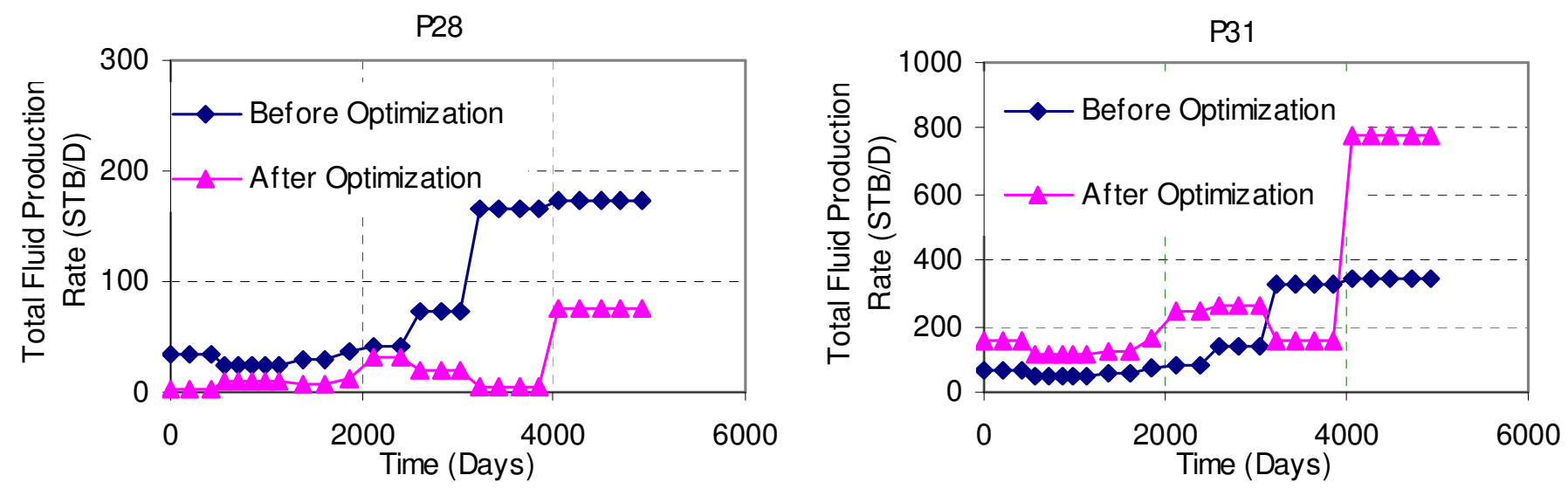

b. Total fluid production rate vs. time

Figure 2.26 Performance plots for P28 and P31 : field example 
It took about 30 minutes to perform the optimization on a Pentium ${ }^{\circledR}$ Dual CPU 3 $\mathrm{GHz}$ desktop. The simulation runs were performed using a two phase streamline simulator.

\subsection{Chapter Summary}

In this chapter we proposed a practical and efficient technique to optimize injection and production rates via flood front management to maximize the sweep efficiency during waterflood. Our work relies on equalizing the arrival times of the waterfront at all producers within selected sub-regions of a water flood project. The approach is computationally efficient and can be applied using both finite-difference and streamline simulators. Some specific conclusions from this study can be summarized as follows:

1. Streamline time of flight provides an effective way to optimize waterflood through equalizing the arrival time at the producers. The streamline approach also facilitates analytical computation of the arrival time sensitivities with respect to production and injection rates.

2. Analytical computation of sensitivities requires only one flow simulation regardless of the number of injectors and producers and leads to an efficient optimization algorithm. The assumptions used to derive the analytical sensitivities were verified by comparing the results with the numerical perturbation method.

3. We have validated our approach using $2 \mathrm{D}$ homogenous and heterogeneous cases and also by application to smart wells with inflow control valves. In all these cases, the optimization has led to a significant delay in water breakthrough with corresponding increase in sweep efficiency and oil recovery.

4. The power and practical feasibility of our method has been demonstrated by a field example from the Goldsmith San Andres Unit (GSAU) in west Texas. We optimized the production rates for 31 wells while accounting for changing field condition such as shut-in and infill drilling.

5. The optimization approach presented here is specific to the geological model used for flow simulation. However, because of its computational efficiency, a 
stochastic approach can be coupled with our method by performing the optimization on multiple realizations and quantifying the uncertainty associated with the optimization result. 


\section{CHAPTER III}

\section{OPTIMAL RATE UNDER GEOLOGIC UNCERTAINTY*}

In this chapter, we propose a practical and efficient method for computing optimal injection and production rates given multiple geologic models. The approach relies on equalizing arrival time of the waterfront at all producers for maximizing the sweep efficiency which was explained in the previous chapter. There are four major characteristics that distinguish our approach from previously published works. First, we use streamlines to efficiently and analytically compute the sensitivity of the arrival times with respect to well rates. Second, we account for geologic uncertainty via two optimization frameworks: (i) a stochastic framework that relies on a combination of the expect value and variance of a performance measure from multiple realizations for risk assessments and (ii) a min-max problem formulation that minimizes the worst case scenario. Third, analytical forms for gradients and Hessian of the objective functions are derived which make our optimization computationally efficient for large field cases. Finally, the optimization is performed under operational and facility constraints using a sequential quadratic programming approach. We propose a measurement-based optimization that allows us to assign specific weights to each realization involved in the optimization process based on observed data.

The organization of this chapter is as follows. First, we outline the major steps of the proposed approach. Next, we discuss the underlying mathematical formulation. These include the objective function formulation, minimization, and measurements-based optimization (MBO). Finally, we demonstrate the power and feasibility of our approach using a $2 \mathrm{D}$ heterogeneous case and a synthetic field example.

\footnotetext{
"Part of this chapter is reprinted with permission from "Optimal Rate Control Under Geologic Uncertainty," by Ahmed H. Alhuthali, Akhil Datta-Gupta, Bevan Yuen, and Jerry P. Fontanilla, paper SPE 113628 presented at the 2008 SPE/DOE Symposium on Improved Oil Recovery, Tulsa, Oklahoma, 20-23 April 2008. Copyright 2008 by the Society of Petroleum Engineers.
} 


\subsection{Approach}

Our approach relies on equalizing arrival time of waterfront at multiple producers to enhance sweep efficiency in a waterflood project accounting for geologic uncertainty. The approach is general and can be implemented using both finite-deference and streamline simulators. We assume that multiple geologic realizations are readily available and the optimization is limited only to wells rates. It doesn't include well placement and configuration as control variable. The major steps of our approach are summarized in Figure 3.1, and are outlined below.

- Flow simulation and streamline tracing. The first step is to conduct flow simulation for each geologic realization. If a finite-difference simulator is used, then we need to trace the streamlines using the fluid fluxes derived from flow simulation (Datta-Gupta and King 2007).

- Compute the travel time residuals, analytical sensitivities, and analytical Jacobian. The second step is to compute the residuals which quantify the misfit between the desired arrival time and the computed arrival time at each producing well for every geologic realization. In addition, this step involves the analytical computation of the arrival time sensitivities for all realizations. The sensitivities are defined as the partial derivatives of arrival time with respect to wells rates. It is important to point out that in our approach the sensitivity computations require only one simulation run per realization and can be carried out simultaneously with the flow simulation. The sensitivities are then used to compute the Jacobian which is defined as the gradient of the residuals.

- Compute Analytical gradient and Hessian. The next step is to use the analytical Jacobian and the residuals to compute the gradient and the Hessian of the objective function. The objective function has a stochastic or a min-max form and will be discussed in detail in the mathematical formulation section.

- Minimization and Optimal Rate Allocations. Once the gradient and the Hessian are available, we can use standard optimization algorithms to minimize the objective function. In our study, we used the Sequential Quadratic Programming (SQP) technique (Nocedal and Wright 2006) to generate the required changes in rates to minimize the objective function subject to appropriate field constraints. 
This step and the above-mentioned steps are repeated until a pre-defined stopping criterion on the objective functions or the rates is met.

- Mobility Effects and Changing Field Conditions. Depending upon the field conditions, occasionally we update the streamlines and perform the optimization again to account for mobility effects and changing field conditions.

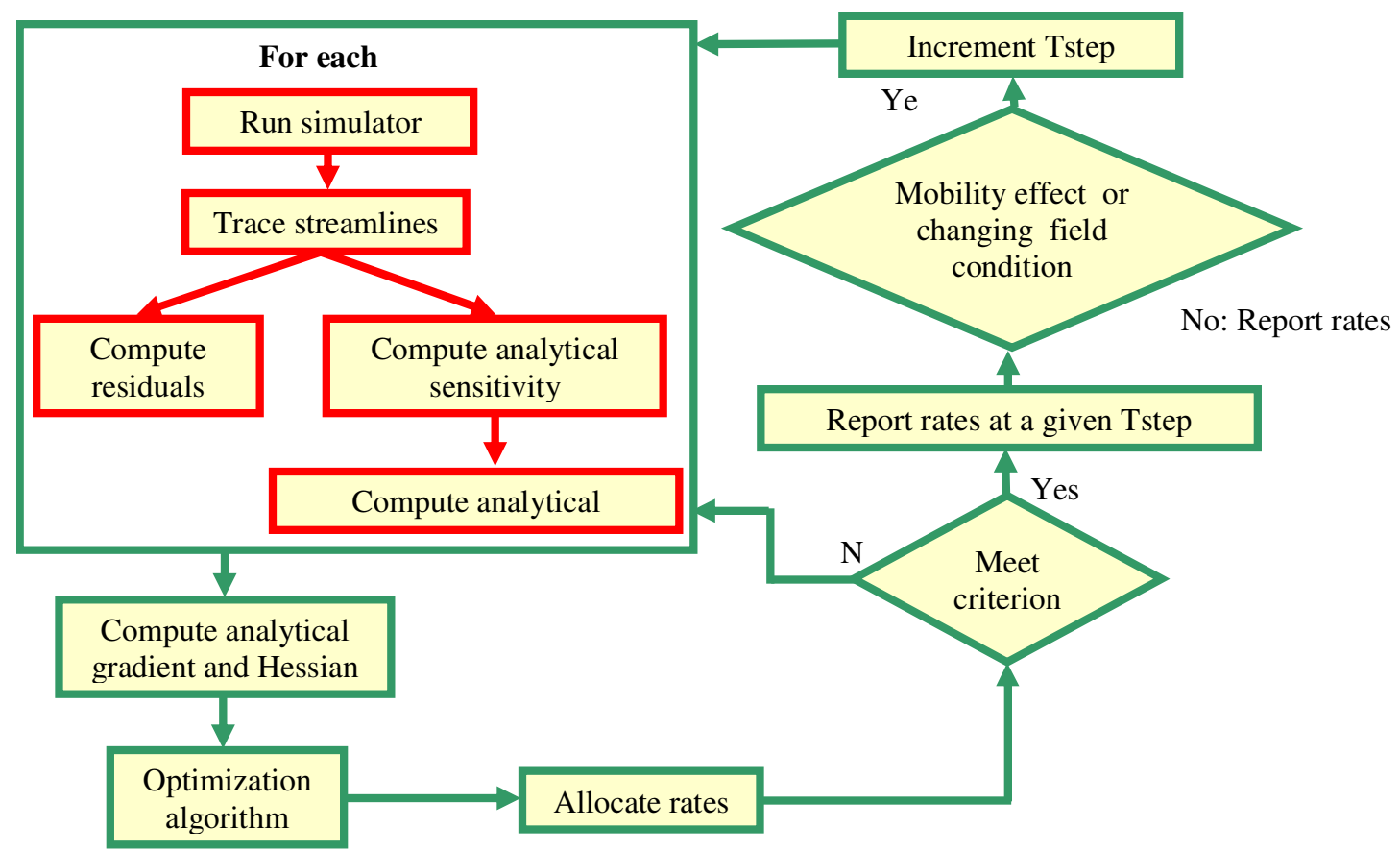

Figure 3.1 Flow chart to show the major step in the optimization approach

\subsection{Mathematical Formulation}

In this section, we discuss the underlying mathematical formulation behind the proposed optimization scheme. First, we present the formulation of the objective function in both stochastic and min-max forms. We then discuss the minimization algorithm and the derivation of the analytical gradient and the Hessian of the objective function. Finally, we discuss the measurements-based optimization to account for field production history. 


\subsubsection{Objective Function Formulation}

Our main objective is to maximize the sweep efficiency in waterflooding project through rate allocation. We accomplish this by equalizing the arrival time of the waterfront at the producers. Mathematically, this requires minimizing an appropriately defined misfit function for a specific group of producers. For a single realization $j$, we can formulate the misfit function as the square of the $l_{2}$ norm of the residuals,

$$
\mathbf{e}_{j}^{T} \mathbf{e}_{j}=\sum_{m=1}^{N_{\text {group }}} \sum_{i=1}^{N_{\text {prod }, m}}\left(t_{d, m}(\mathbf{q})-t_{i, m}(\mathbf{q})\right)^{2}
$$

The arrival time residuals are represented by the vector e in Eq. 3.1. The variable $t_{i, m}$ represents the calculated arrival time at well $i$, belonging to group $m$. The desired arrival time, $t_{d, m}$ for the well group $m$ is given by the arithmetic average of $t_{i, m}$ for each iteration during the optimization. The vector $\mathbf{q}$ contains the control variables and has a dimension of $n$, the number of well rates to be optimized.

To address geologic uncertainty, Eq. 3.1 needs to be generalized to handle multiple realizations. In this paper, we will use two forms of the objective function to address uncertainty. The first one is a stochastic formulation in terms of an expected value of the misfit in Eq. 3.1 for multiple realizations penalized by its standard deviation as follows,

$$
f(\mathbf{q})=\mathrm{E}\left[\mathbf{e}^{T} \mathbf{e}\right]+r \sigma\left[\mathbf{e}^{T} \mathbf{e}\right]
$$

Equation 2 can derived within the decision analysis framework as shown in AppendixA. The decision analysis framework and concepts have been used previously in the oil industry (Bickel et. al. 2006; Guyaguler and Horne 2001; Simpson et. al. 2000; Yeten et. al. 2003). The variable $r$ is the risk coefficient that weights the trade off between the expected value and the standard deviation. A positive $r$ means that the decision maker is risk averse, while a negative $r$ means that the decision maker is risk prone. A zero risk coefficient indicates that the decision maker is risk neutral. In this study we will limit our 
discussions to positive and zero risk attitude coefficients. The expected value and the standard deviation are given by the following equations,

$$
\begin{aligned}
& \mathrm{E}\left[\mathbf{e}^{T} \mathbf{e}\right]=\frac{1}{N_{r}} \sum_{i=1}^{N r} \mathbf{e}_{i}^{T} \mathbf{e}_{i} \\
& \sigma\left[\mathbf{e}^{T} \mathbf{e}\right]=\sqrt{\operatorname{Var}\left(\mathbf{e}^{T} \mathbf{e}\right)}=\left[\mathrm{E}\left[\left(\mathbf{e}^{T} \mathbf{e}\right)^{2}\right]-\mathrm{E}^{2}\left[\mathbf{e}^{T} \mathbf{e}\right]\right]^{1 / 2}
\end{aligned}
$$

The variable $N_{r}$ in Eq. 3.3 refers to the number of realizations used in the optimization. Our goal is to minimize Eq. 3.2 by changing $\mathbf{q}$, the injection/production rates, subject to multiple equality and inequality constraints imposed by the operational restrictions and facility limitations. Thus,

$$
\min _{\mathbf{q}} f(\mathbf{q})
$$

Subject to

$$
\begin{aligned}
& \mathbf{h}(\mathbf{q})=0 \\
& \mathbf{g}(\mathbf{q}) \leq 0
\end{aligned}
$$

Where $h: \mathfrak{R}^{n} \rightarrow \mathfrak{R}^{z}$ and $g: \mathfrak{R}^{n} \rightarrow \mathfrak{R}^{y}$

The superscripts $z$ and $y$ represent the number of equality and inequality constraints respectively.

The second form of the objective function involves a min-max optimization scheme. This approach attempts to optimize the worst-case scenario. The problem can be formulated as follows (Brayton et al. 1979),

$$
\min _{\mathbf{q}} \max _{i} \mathbf{e}_{i}^{T} \mathbf{e}_{i}
$$

Subject to

$$
\begin{aligned}
& \mathbf{h}(\mathbf{q})=0 \\
& \mathbf{g}(\mathbf{q}) \leq 0
\end{aligned}
$$

Where $h: \mathfrak{R}^{n} \rightarrow \mathfrak{R}^{z}$ and $g: \mathfrak{R}^{n} \rightarrow \mathfrak{R}^{y}$ 
For our application, we assume that the constraints are linear and they have the following forms:

$$
\begin{aligned}
& \mathbf{h}(\mathbf{q})=\mathbf{A q}+\mathbf{b} \\
& \mathbf{g}(\mathbf{q})=\mathbf{C q}+\mathbf{d}
\end{aligned}
$$

\subsubsection{Objective Function Minimization}

In this section, we discuss the approaches to minimize Eq. 3.4 and 3.5. Equation 4 can be minimized using the sequential quadratic programming (SQP) algorithm for nonlinear constrained optimization (Nocedal and Wright 2006). The main concept behind the approach is to formulate the problem into a series of quadratic programming (QP) sub-problems which can be solved at each major iteration $k$. The QP sub-problem is mainly a quadratic approximation of the Lagrangian of Eq. 3.4 which is given in the following form:

$$
L\left(\mathbf{q}, \lambda_{L}, \lambda_{K}\right)=f(\mathbf{q})+\lambda_{L}^{T} \mathbf{h}(\mathbf{q})+\lambda_{K}^{T} \mathbf{g}(\mathbf{q})
$$

The vectors $\lambda_{L}$ and $\lambda_{K}$ refer to the Lagrange multipliers corresponding to the equality constraints and the Karush-Kuhn-Tucker multipliers corresponding to the inequality constraints. After linearizing the constraints using a Taylor approximation, the QP subproblem can be written as:

$$
\min _{\delta \mathbf{q}} f\left(\mathbf{q}^{k}\right)+\nabla_{q} f^{T}\left(\mathbf{q}^{k}\right) \delta \mathbf{q}+\frac{1}{2} \delta \mathbf{q} \nabla_{q}^{2} L\left(\mathbf{q}^{k}\right) \delta \mathbf{q}
$$

Subject to

$$
\begin{aligned}
& \mathbf{h}\left(\mathbf{q}^{k}\right)+\nabla_{q} \mathbf{h}\left(\mathbf{q}^{k}\right)^{T} \delta \mathbf{q}=0 \\
& \mathbf{g}\left(\mathbf{q}^{k}\right)+\nabla_{q} \mathbf{g}\left(\mathbf{q}^{k}\right)^{T} \delta \mathbf{q} \leq 0
\end{aligned}
$$

Equation 8 indicates that we need to evaluate the following terms at each iteration to minimize Eq. 3.4:

- The objective function : $f\left(\mathbf{q}^{k}\right)$

- The gradient of the objective function : $\nabla_{q} f\left(\mathbf{q}^{k}\right)$ 
- The Hessian of the Lagrangian: $\nabla_{q}^{2} L\left(\mathbf{q}^{k}\right)$. This term is equal to the Hessian of the objective function, $\nabla_{q}^{2} f\left(\mathbf{q}^{k}\right)$, given that the constraints are linear.

- The gradient of the constraints: $\nabla_{q} \mathbf{h}\left(\mathbf{q}^{k}\right)$ and $\nabla_{q} \mathbf{g}\left(\mathbf{q}^{k}\right)$

The objective function evaluation relies mainly on the computation of the residuals which we will discuss later. The gradient of the constraints are straightforward because we assumed that they are linear with respect to the control variable. The computation of the gradient and Hessian of the objective function will be discussed in the next section.

The second form of the objective function that we are interested in is a min-max problem given by Eq. 3.5. This problem can be reformulated into an equivalent linear programming problem as follows (Brayton et. al. 1979).

$$
\begin{array}{cl}
\underset{\mathbf{q}}{\min } \gamma & \\
\text { Subject to } & \\
& f_{i}(\mathbf{q}) \leq \gamma \\
& \mathbf{h}(\mathbf{q})=0 \\
& \mathbf{g}(\mathbf{q}) \leq 0
\end{array}
$$

Where $h: \mathfrak{R}^{n} \rightarrow \mathfrak{R}^{z}$ and $g: \mathfrak{R}^{n} \rightarrow \mathfrak{R}^{y}$

$f_{i}(\mathbf{q})$ is equal to $\mathbf{e}_{i}^{T} \mathbf{e}_{i}$ which represents the residuals obtained from realization $i$ and will be treated as a constraint in Eq. 3.9. It will be approximated using Taylor series expansion.

$$
f_{i}(\mathbf{q}+\delta \mathbf{q})=f_{i}(\mathbf{q})+\nabla^{T} f_{i}(\mathbf{q}) \delta \mathbf{q}
$$

This requires the computation of the gradient of $f_{i}(\mathbf{q})$ for each realization involved in the optimization. The gradient can be evaluated using the following expression:

$$
\nabla_{q} f_{i}(\mathbf{q})=\nabla_{q}\left[\mathbf{e}_{i}^{T} \mathbf{e}_{i}\right]=2 \mathbf{J}_{i}^{T} \mathbf{e}_{i}
$$


The term $\mathbf{J}_{\mathrm{i}}$ refers to the Jacobian Matrix for realization $i$. Later we will show that the Jacobian Matrix can be computed analytically without additional simulation runs.

\subsubsection{Objective Function Evaluation}

The objective function evaluation in Eq. 3.8 and 3.9 relies on the computation of the residuals for each realization. We introduced the general form for residuals in Eq. 3.1, but we haven't explained how to calculate the waterfront arrival time and the desired arrival time.

The calculated arrival time is given by the following expression.

$$
t_{i, m}(\mathbf{q})=\frac{1}{N_{f s l, i}} \sum_{l=1}^{N_{f s l, i}} \tau_{l, i} /\left[\frac{d f_{w}}{d S_{w}}\right]_{S_{w}=S_{w f, l}}
$$

In the above expression, $N_{f s, i}$ represents the number of the fast streamlines connected to the producer $i$ belonging to group $m$. We choose a set of the fast streamlines which consists of a fraction of $N_{d, i}$, the total number of streamlines connected to the producer $i$. The variable $\tau$ represents the usual streamline time-of-flight. The desired arrival time for a group of producers is computed as the arithmetic average of the calculate arrival times at the producers within this group.

$$
t_{d, m}(\mathbf{q})=\frac{\sum_{i}^{N_{p r o d, m}} t_{i, m}(\mathbf{q})}{N_{p r o d, m}}
$$

In the previous chapter, we provided a complete discussion on the derivation of Eq. 3.12 and 3.13.

\subsubsection{Objective Function Gradients and Hessian}

The gradient of the objective function in Eq. 3.2 is given by the following expression: 


$$
\nabla_{q} f(\mathbf{q})=2 \mathrm{E}\left[\mathbf{J}^{T} \mathbf{e}\right]+2 r \frac{\operatorname{Cov}\left(\mathbf{e}^{T} \mathbf{e}, \mathbf{J}^{T} \mathbf{e}\right)}{\sigma\left(\mathbf{e}^{T} \mathbf{e}\right)}
$$

The derivation of Eq. 3.14 is given in Appendix-B. The first term in Eq. 3.14, E[J $\mathbf{J}^{\mathrm{T}} \mathbf{e}$, represents the expected value of the gradient of the misfit function in Eq. 3.1 computed for each realization. The second term contains $\operatorname{Cov}\left(\mathbf{e}^{\mathrm{T}} \mathbf{e}, \mathbf{J}^{\mathrm{T}} \mathbf{e}\right)$ which represents the crosscovariance vector of the misfit and its gradient computed for each realization. For illustration, a single element with an index $i$ in the cross covariance vector is given by the following expression:

$$
\operatorname{Cov}_{i}=\operatorname{Cov}\left(\mathbf{e}^{T} \mathbf{e},\left(\mathbf{J}^{T} \mathbf{e}\right)_{i}\right)
$$

The expression in Eq. 3.15 indicates that an element $i$ can be obtained by computing then cross covariance between two vectors. The first term is the misfit function in Eq. 3.1 for each realization and the second term is the $i^{\text {th }}$ element of the gradient evaluated for each realization.

The Hessian of the objective function in Eq. 3.2 is given by,

$$
\begin{aligned}
\nabla_{q}^{2} f(\mathbf{q})=2 \mathrm{E}\left[\mathbf{J}^{T} \mathbf{J}\right] & +r \frac{4 \operatorname{Cov}\left(\mathbf{J}^{T} \mathbf{e}, \mathbf{J}^{T} \mathbf{e}\right)+2 \operatorname{Cov}\left(\mathbf{e}^{T} \mathbf{e}, \mathbf{J}^{T} \mathbf{J}\right)}{\sigma\left(\mathbf{e}^{T} \mathbf{e}\right)} \\
& -r \frac{4 \operatorname{Cov}\left(\mathbf{e}^{T} \mathbf{e}, \mathbf{J}^{T} \mathbf{e}\right) \operatorname{Cov}^{T}\left(\mathbf{e}^{T} \mathbf{e}, \mathbf{J}^{T} \mathbf{e}\right)}{\sigma^{3}\left(\mathbf{e}^{T} \mathbf{e}\right)}
\end{aligned}
$$

The derivation of Eq. 3.16 is presented in Appendix-C. The first term, $E\left[\mathbf{J}^{\mathrm{T}} \mathbf{J}\right]$ is the expected value of Hessian of the square of the $l_{2}$ norm of the residuals computed for each realization. $\operatorname{Cov}\left(\mathbf{J}^{\mathrm{T}} \mathbf{e}, \mathbf{J}^{\mathrm{T}} \mathbf{e}\right)$ is the covariance matrix for the gradients vectors obtained from each realization. A single element in this matrix can be obtained using the following expression:

$$
\operatorname{Cov}_{i j}=\operatorname{Cov}\left(\left(\mathbf{J}^{T} \mathbf{e}\right)_{i},\left(\mathbf{J}^{T} \mathbf{e}\right)_{j}\right)
$$


This expression means that an $i j$-element where $i$ is the row index and $j$ is the column index, is evaluated by computing the covariance between two series of numbers. The first one represents the $i^{\text {th }}$ element of each gradient vector, and the second series represents the $j^{\text {th }}$ element of each gradient vector. $\operatorname{Cov}\left(\mathbf{e}^{\mathrm{T}} \mathbf{e}, \mathbf{J}^{\mathrm{T}} \mathbf{J}\right)$ is a cross covariance matrix between the square of the $l_{2}$ norm of the residuals evaluated for each realization and the Hessian of the $l_{2}$ norm of the residuals computed for each realization. An element in this matrix, $\operatorname{Cov}_{i j}$, is computed using the following expression:

$$
\operatorname{Cov}_{i j}=\operatorname{Cov}\left(\mathbf{e}^{T} \mathbf{e},\left(\mathbf{J}^{T} \mathbf{J}\right)_{i j}\right)
$$

The above expression indicates that a single element located at $i j$-index is computed by a cross-covariance between two series of numbers. The first series is the square of the $l_{2}$ norm for each realization, and the second one is the $i j$-elements of the Hessian matrix computed for each realization.

\subsubsection{Jacobian Matrix and Analytical Sensitivity Calculations}

In this section, we show the computation of the Jacobian matrix, J, for each realization. The Jacobian is given by the following expression:

$$
\mathbf{J}=\nabla_{q} \mathbf{e}
$$

A single element in the residual vector, e, can be written as follows:

$$
e_{i, m}=t_{d, m}(\mathbf{q})-t_{i, m}(\mathbf{q})
$$

Equation 20 refers to the arrival time residual at producer $i$. By combining Eq. 3.13, 3.19, and 3.20 It can be shown that a single element in the Jacobian matrix can be written as,

$$
J_{i j}=\left[\frac{1}{N_{\text {Group }}} \sum_{k=1}^{N_{\text {Group }}} S_{k j}\right]-S_{i j}
$$


The sensitivity coefficient $S_{i j}$ quantifies the changes in arrival time at producer $i$ because of small changes in the rate of well $j$. It is given by

$$
S_{i j}=\frac{\partial t_{i, m}(\mathbf{q})}{\partial q_{j}}
$$

In the previous chapter, we derived an analytical form for these sensitivities. If the derivative is taken with respect to the rate of a producer, the sensitivity coefficient is given by the following expression:

$$
\begin{aligned}
& S_{i j}=-\frac{t_{i, m}}{q_{j}} \quad \forall i=j \\
& S_{i j}=0 \quad \forall i \neq j \\
& j \text { is a producer }
\end{aligned}
$$

In Eq. 3.22, we assume that $t_{i, m}$ is sensitive only to the production of producer $i$. The sensitivity of $t_{i, m}$ is considered to be negligible with respect to the rates of other producers.

If the derivative is taken with respect to the rate of an injector, the sensitivity coefficient can be written as follows:

$$
\begin{aligned}
& S_{i j}=-\frac{\sum_{l=1}^{N_{f l l i, j}} \tau_{l, i, j} /\left[\frac{d f_{w}}{d S_{w}}\right]_{S_{w}=S_{w f, l}}}{q_{j} N_{f s l, i}} \text { if } N_{f s l, i, j} \neq 0 \\
& S_{i j}=0 \text { if } N_{f s l, i, j}=0 \\
& j \text { is an injector }
\end{aligned}
$$

The variable $N_{f s l, i, j}$ is the number of the fast streamlines connecting a producer $i$ to an injector $j$. This number represents only a fraction of $N_{f s l, i}$ the total number of the fastest streamlines connected to the producer $i$. If the injector $j$ is not connected to producer $i$ through a fast streamline i.e. $\left(N_{f s l, i, j}=0\right)$, then the arrival time at producer $i$ is not sensitive to a perturbation in the rate of injector $j$. 
The analytical calculation of sensitivities leads to an analytical calculation of the Jacobian matrix for each realization. Consequently, this leads to an analytical calculation of the gradient and Hessian of objective function as shown by Eq. 3.14 and 3.16. Also, it leads to an analytical calculation of the gradient to solve the min-max problem shown by

\section{Eq. 3.11.}

It is important to emphasize that the computation of the Jacobian matrix requires a single flow simulation using either a streamline or a finite-difference simulator. On the other hand, if we compute the Jacobian matrix by numerical perturbation, it will require $1+N_{p r o d}+N_{i n j}$ simulation runs if both production/injection rates were optimized. Thus, analytical computation of Jacobian matrix can lead to a substantial savings in computation time.

\subsubsection{Measurements-Based-Optimization}

In this section, we will discuss how to include observed measurements in the optimization process if they are available. Unlike some previous approaches where measurements are used to history match the reservoir model, we propose a method to incorporate production data in the optimization by giving different weight to multiple realizations. Previously, we assumed that all realizations are equiprabable and has equal weights, $1 / N_{r}$, which is implied by Eq. 3.3. The question is "can we assign different weights for each realizations based on their closeness to the observed data?" Under such conditions, the expected value of the square of $l_{2}$ norms of the residuals will be given as follows,

$$
\begin{aligned}
& \mathrm{E}\left[\mathbf{e}^{T} \mathbf{e}\right]=\sum_{i=1}^{N r} w_{i}\left[\mathbf{e}_{i}^{T} \mathbf{e}_{i}\right] \\
& \sum_{i=1}^{N r} w_{i}=1
\end{aligned}
$$

This can be achieved through the application of the Bayes' theorem. Let's denote $P\left(X_{\mathrm{i}}\right)$ as the prior distribution of model parameters $X_{i}$. Also, let's denote $P\left(d \mid X_{i}\right)$ as the likelihood of the observed measurements $d$ given that we have a reservoir model $X_{i}$. The weight $w_{i}$, can be written as 


$$
w_{i}=P\left(X_{i} \mid d\right)=\frac{P\left(X_{i}\right) P\left(d \mid X_{i}\right)}{\sum_{j=1}^{N_{r}} P\left(X_{j}\right) P\left(d \mid X_{j}\right)}
$$

In the above expression, we assign weights based on a conditional probability of the model parameters given that we have measurements available. If we assume that the prior models are equiprobable, we can write the weights in terms of the likelihoods only.

$$
w_{i}=\frac{P\left(d \mid X_{i}\right)}{\sum_{j=1}^{N_{r}} P\left(d \mid X_{j}\right)}
$$

The likelihoods represent the distribution of errors between the observed measurements and the simulation response $g\left(X_{i}\right)$. The errors are assumed to be additive and Gaussian with zero mean and covariance $C_{d}$.

$$
P\left(d \mid X_{i}\right) \alpha \operatorname{Exp}\left(-\frac{1}{2}\left(d-g\left(X_{i}\right)\right)^{T} C_{d}^{-1}\left(d-g\left(X_{i}\right)\right)\right)
$$

By substituting Eq. 3.28 into Eq. 3.27, we can write the weights as follows,

$$
w_{i}=\frac{\operatorname{Exp}\left(-\frac{1}{2}\left(d-g\left(X_{i}\right)\right)^{T} C_{d}^{-1}\left(d-g\left(X_{i}\right)\right)\right)}{\sum_{j=1}^{N_{r}} \operatorname{Exp}\left(-\frac{1}{2}\left(d-g\left(X_{j}\right)\right)^{T} C_{d}^{-1}\left(d-g\left(X_{j}\right)\right)\right)}
$$

The above expression is general enough to include all kind of measurements. In this paper, we will present an example of including 4D seismic data in the optimization process. It is important to point out that the weights can be continuously updated as more measurements become available.

\subsection{Applications and Discussion}

In this section, we first use a 2D heterogeneous example to illustrate our approach for addressing geologic uncertainty. Next we demonstrate the practical viability of the approach using a synthetic field example based on a giant middle-eastern field. Finally, 
we revisit the $2 \mathrm{D}$ heterogeneous case to illustrate the impact of incorporating observed measurements in the optimization scheme and how that can facilitate decision-making.

\subsubsection{Illustration of the Approach: A 2 D Heterogeneous Example}

This example case involves four producers and one injector arranged in a fivespot pattern (Figure 3.2). The porosity is fixed at 0.225 and the permeability is spatially heterogeneous. The reservoir has a dimension of $1716 \times 1716 \times 33 \mathrm{ft}$ and is discretized into $50 \times 50 \times 1$ grid blocks. The field total production rate equals to $2520 \mathrm{RB} / \mathrm{D}$. For the base case, we divide the total production equally among all producers. The optimization is performed only on the producers under the following constrains: (i) the total production should be kept the same (ii) the maximum individual well total production is $1100 \mathrm{RB} / \mathrm{D}$ and (iii) the minimum individual well total production is $150 \mathrm{RB} / \mathrm{D}$. We generated 182 realizations of the permeability field out of which 32 realizations are used for the optimization and the rest for validation purposes. A sample of the 32 realization is shown in Figure 3.3. These realizations were generated using sequential Gaussian simulation with strongly anisotropic variograms.

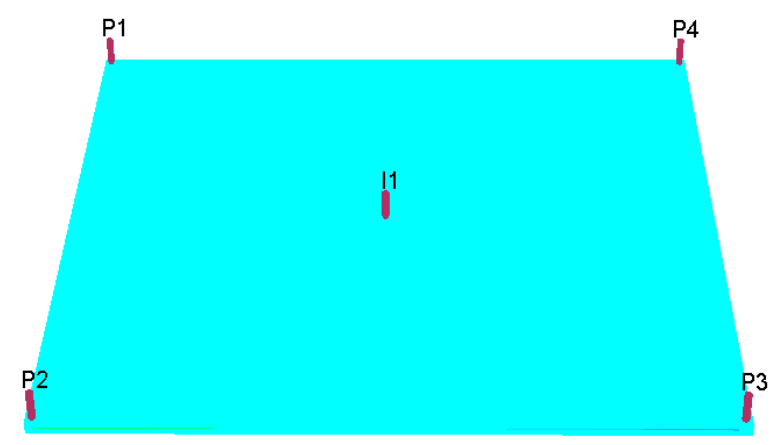

Figure 3.2 Well locations: 2D heterogeneous case 

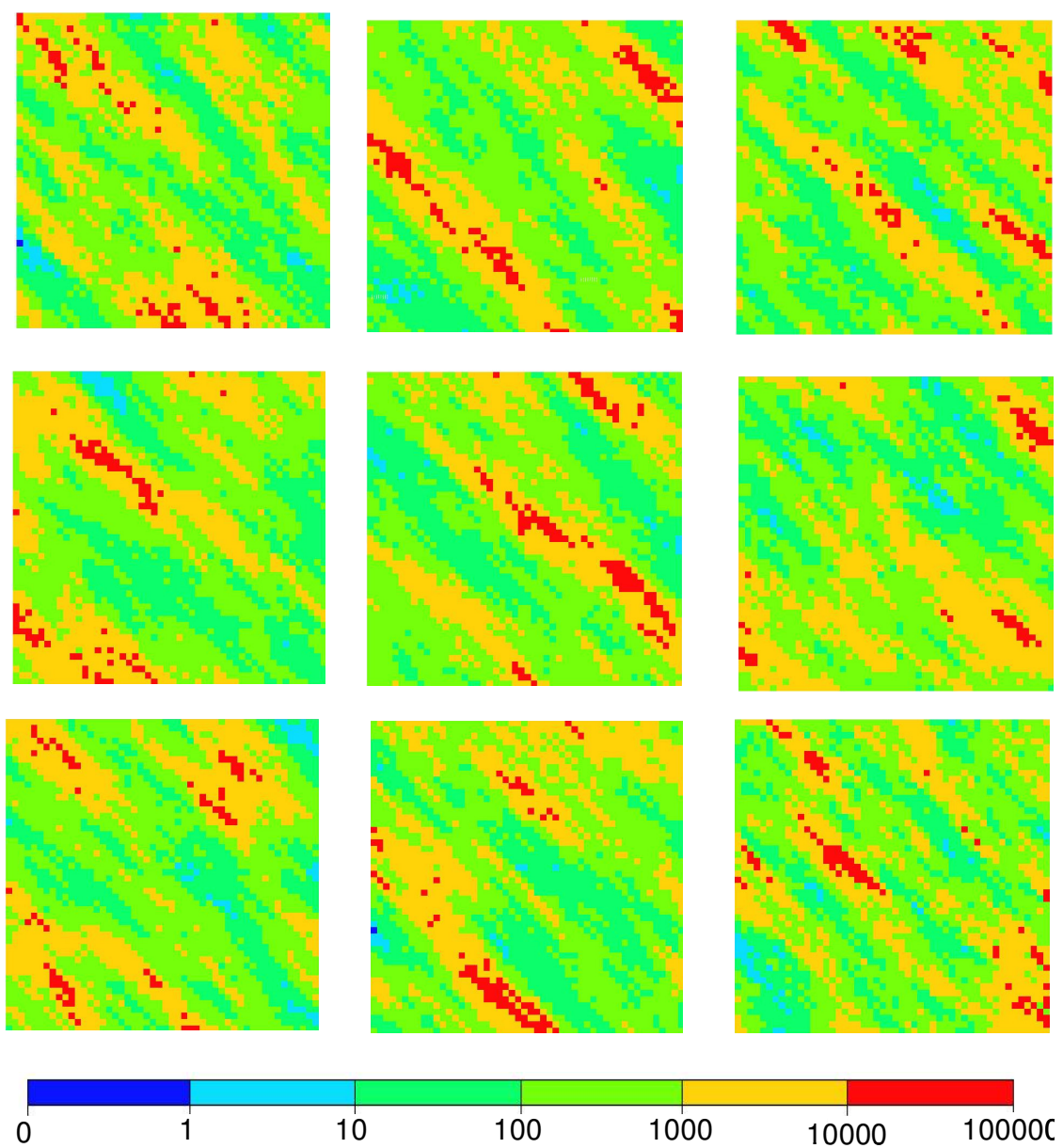

Figure 3.3 Multiple realizations of permeability $(\mathrm{md}): 2 \mathrm{D}$ heterogeneous case

First, let's start with examining the impact of different number of realizations on the optimization. We assume to be risk-neutral $(r=0)$ and perform the optimization using the following number of realizations: 1, 2, 4, 8, 16, and 32. The total well fluid rates after optimization are shown in Figure 3.4. Although the rates obtained from each optimization case are different, the general trends of high and low productions are the same for all cases. It is somewhat expected because all the realizations were drawn from the same distribution and they have similar permeability trends. 


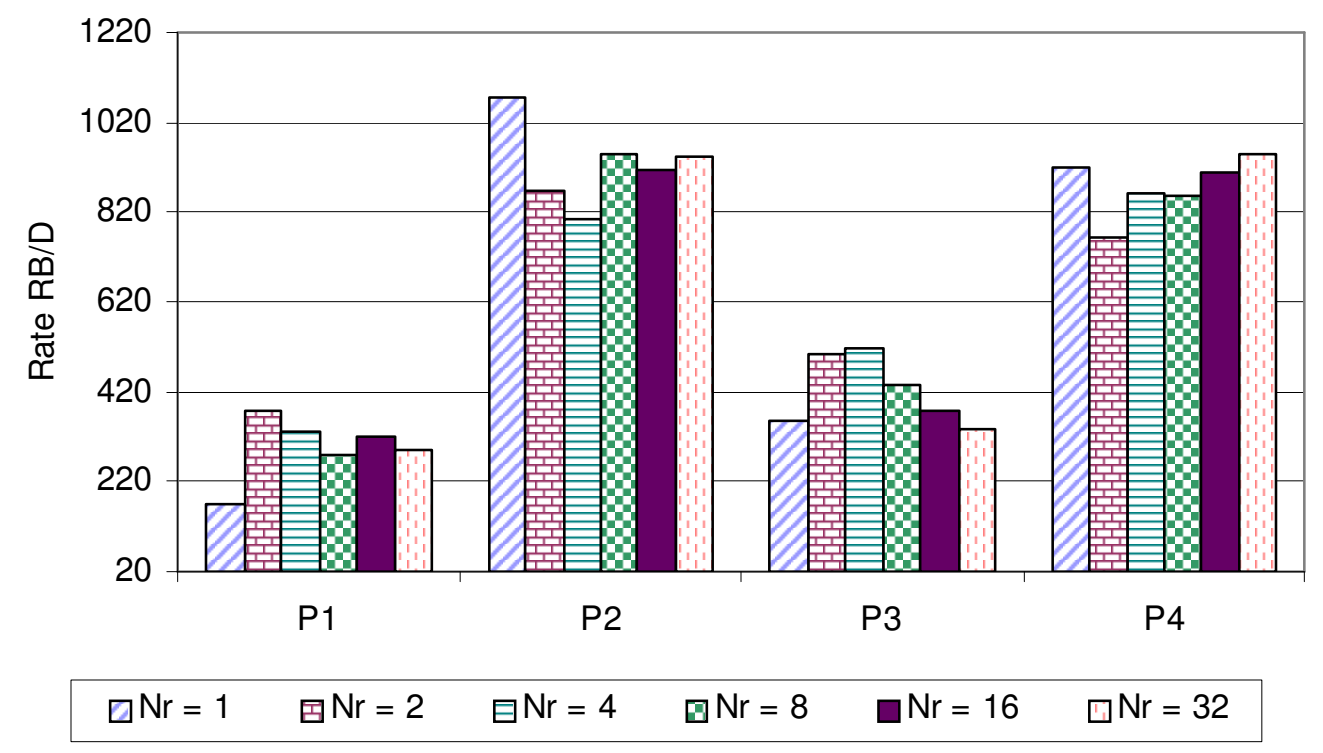

Figure 3.4 Optimized rates using different number of realizations (Nr): 2D heterogeneous case

The impact of the optimization on the expected value and the standard deviation of the arrival time misfit is shown Figure 3.5. Here we have shown a comparison between the base case and the optimized case with 32 realizations. For this, we run the flow simulator for each of the 32 realizations with the base case rates and the rates obtained from the optimized case with 32 realizations. The results show that with the optimized rates we manage to reduce the expected value by almost $67 \%$ and the standard deviation by almost 58\%. It is important to point out that the huge improvement in the standard deviation was achieved although we set $r$ to be zero. This seems to indicate that the expected value and the standard deviation are highly correlated for the case studied. 


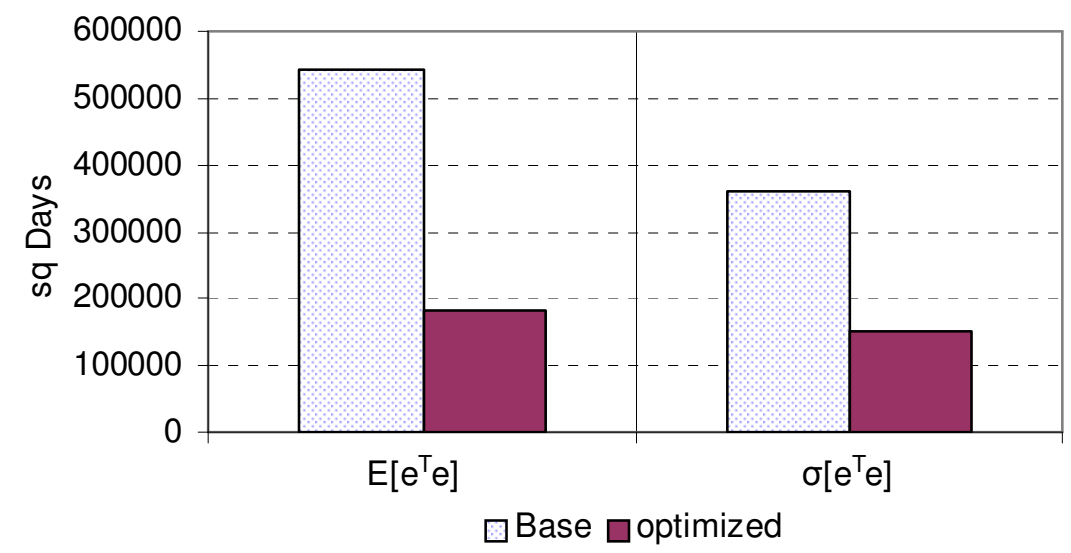

\section{Figure 3.5 Comparison between the base case and the optimized case in terms of expected value and standard deviation: $2 \mathrm{D}$ heterogeneous case, $\mathrm{Nr}=32$}

We performed additional sensitivity on the choice of $r$. Here, we fix the number of permeability realizations at 32 and choose the following value of $r$ to be: $0,1,2,3$, and 4. Recall that positive values of $r$ indicate risk averse attitude. We will compare the results with different risk attitude coefficients. We will also compare these results to the min-max optimization which is actually optimizing the worst case scenario. For this, we first perform the stochastic optimization with multiple values of $r$ as well as the min-max optimization using the 32 realizations. Next, we run the forward simulation for the 32 realizations using the rates obtained from these different optimization cases. The travel time residuals for each realization are then used to compute the expected value and the standard deviation. Figure 3.6 shows the comparison in terms of the expected value. As $r$ increases, the expected value of travel time misfit increases because of the trade-off with the standard deviation. The min-max case, representing optimizing the worst case scenario has the highest expected value but still lower than the base case in Figure 3.5. Figure 3.7 shows that as $r$ increases the standard deviation decreases as one might expect from Eq. 3.2. The standard deviation for the min-max case is only slightly lower than the case with $r$ equal to zero for this example. 


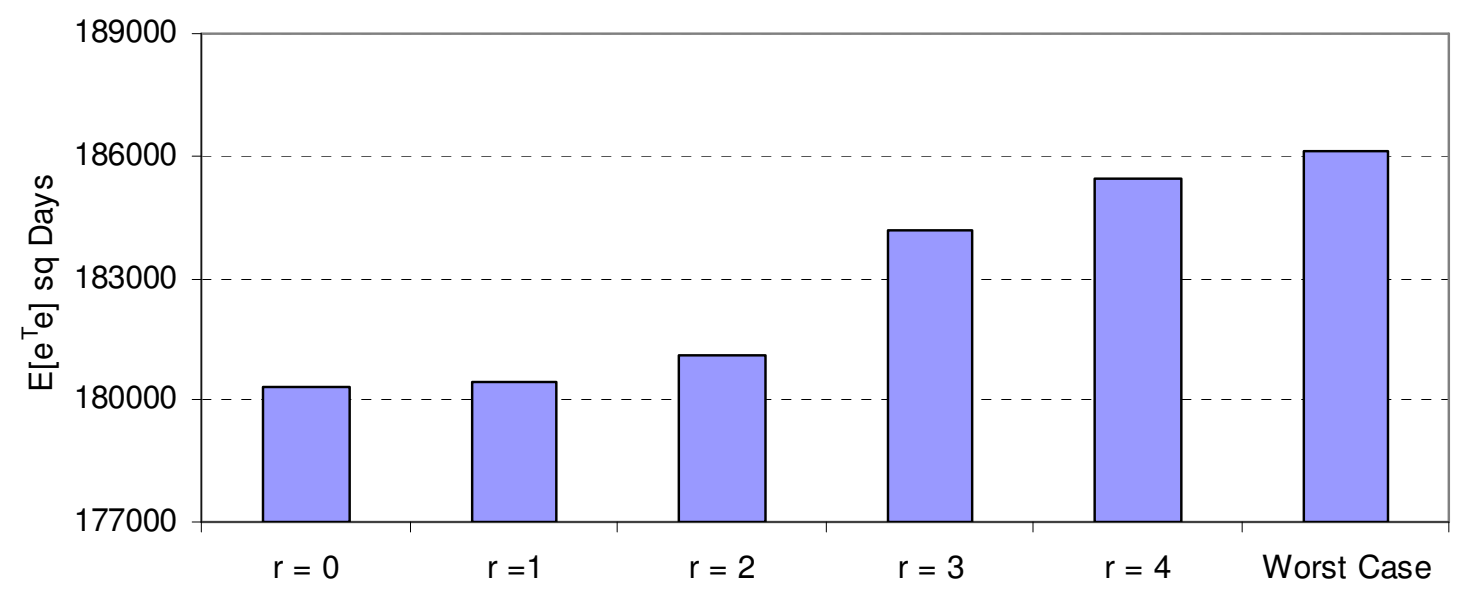

Figure 3.6 Comparison in terms of the expected value for different $r$ and the worst case scenario after optimization: $2 \mathrm{D}$ heterogeneous case, $\mathrm{Nr}=32$

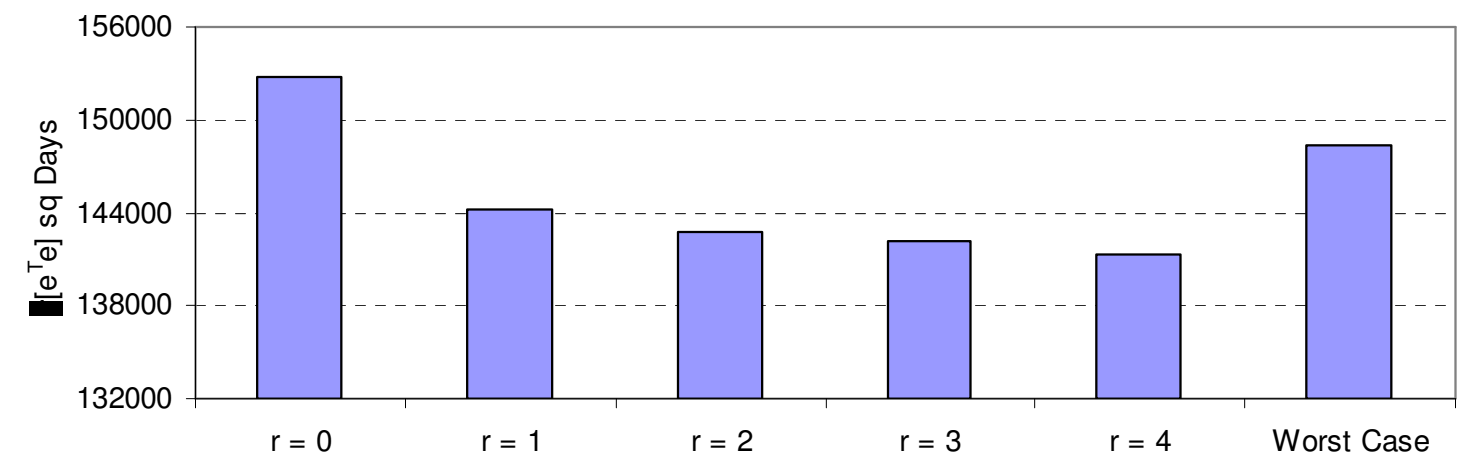

Figure 3.7 Comparison in terms of the standard deviation for different $r$ and the worst case scenario after optimization: $2 \mathrm{D}$ heterogeneous case, $\mathrm{Nr}=32$

So far, we have seen the results of the stochastic and the min-max optimization in terms of the expected value of the arrival time misfit and its standard deviation from 32 realizations. However, the min-max optimization does not address the expected value; rather, it tries to improve the worst case scenario. This is reflected in Figure 3.8 which compares the maximum of arrival time misfit for 32 realizations after optimizing with different $r$ and the min-max case. The min-max case which optimizes the worst case 
scenario has the lowest value, as expected. However, all the optimized cases have arrival time misfit values that are lower than that of the base (un-optimized) case (1694370 Sq. days).

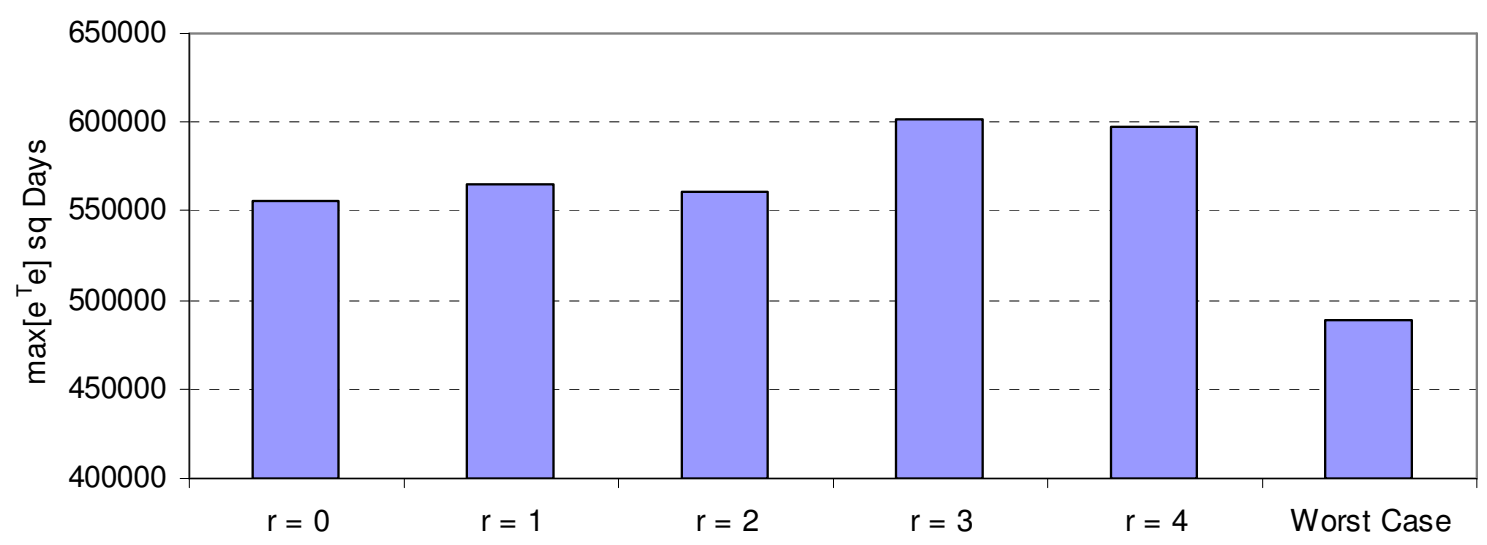

Figure 3.8 Comparison in terms of the maximum of the square of $\mathbf{l}_{\mathbf{2}}$ norm of residuals for different $\boldsymbol{r}$ and the worst case scenario after optimization : 2D heterogeneous case, $\mathrm{Nr}=32$

Finally, we examine the impact of optimization on the sweep efficiency. Recall that the objective of our optimization is to maximize waterflood sweep efficiency and hence increase oil production and recovery. We now use the 150 realizations that were set aside in the beginning. Specifically, we run the flow simulator for 1500 days for these 150 realizations using the total fluid production rates obtained from the following cases:

- No control: the production wells are produced based on their productivity index.

- Optimized case using 32 realization and $\mathrm{r}=0$.

- Optimized case using single realization.

- Based case: equal rates.

We will present the results in terms of average cumulative oil production from 150 realizations for each of the above cases. Figure 3.9 shows a significant increase in 
average cumulative oil production obtained from the optimized case using 32 realizations and $r=0$ compared to the case with no control. These results clearly show the value of control in waterflood projects. Figure 3.10 shows that the optimized cases will yield higher average cumulative oil production compared to the base case with equal rates for all producers. More importantly, it shows that the optimized case using 32 realizations will yield a higher average cumulative oil production compared to the optimized case using the single realization chosen here. These results underscore the value of addressing geologic uncertainty in the optimization process.

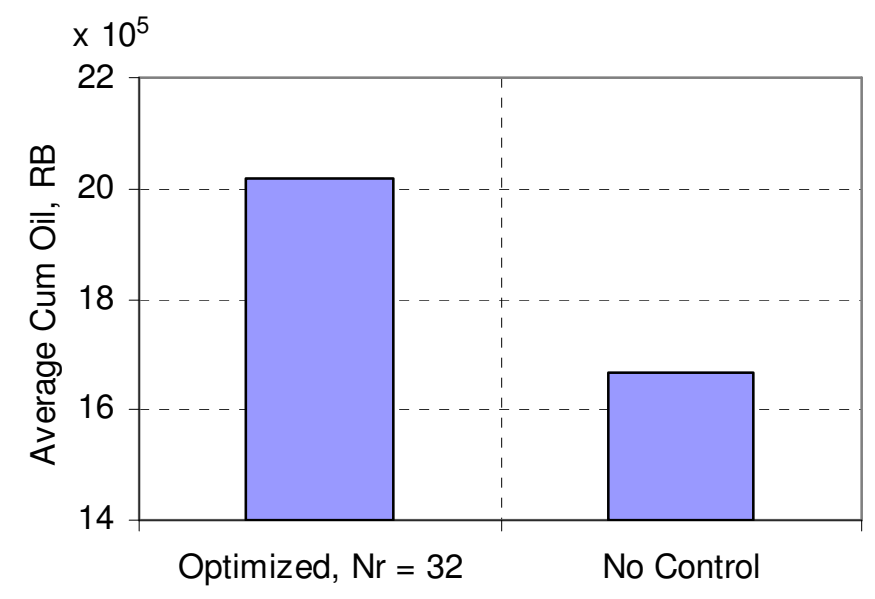

Figure 3.9 Comparison between the optimized case using 32 realizations and no control case in terms of the average cumulative oil production from 150 realizations : 2D heterogeneous case, $r=0$ 


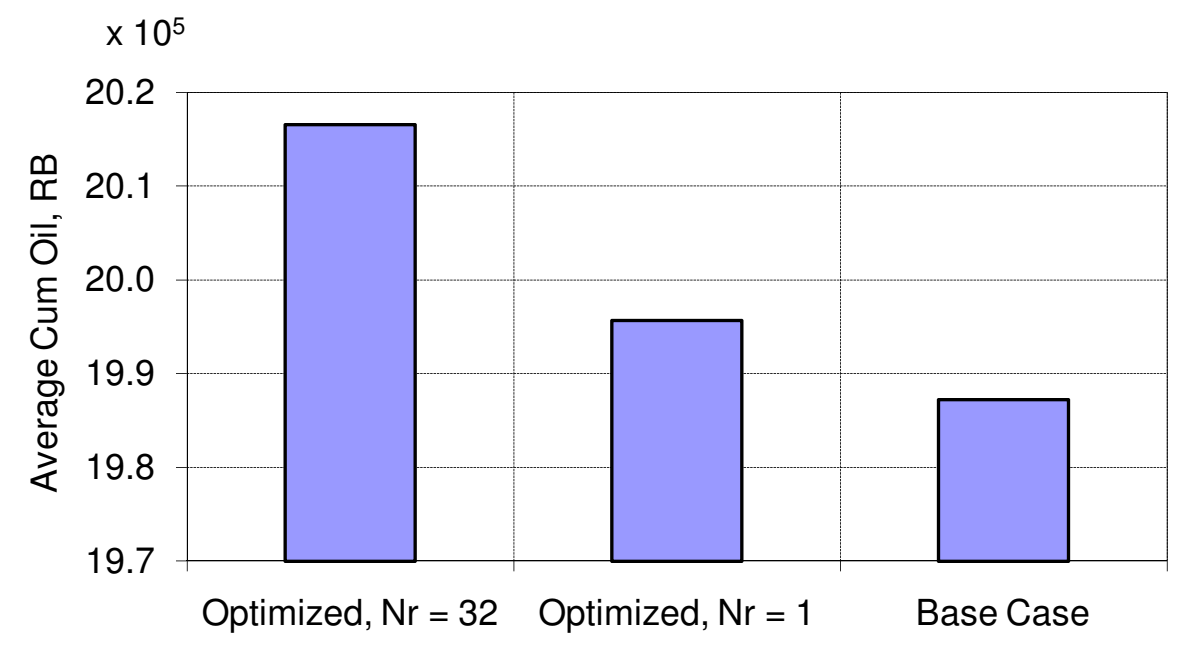

Figure 3.10 Comparison between the optimized case using 32 realization, the optimized case using a single realization, and the base case in terms of the average cumulative oil production from 150 realizations : 2D heterogeneous case, $r=0$

\subsubsection{A Synthetic Field Example}

We have applied our approach to a synthetic field example based on a giant middle-eastern field. This field has more than four oil and gas bearing reservoirs. The reservoir under study is a folded anticline consisting primarily of Jurassic carbonate. Matrix porosity and permeability averaged $25 \%$ and $600 \mathrm{md}$, respectively (Alhuthali et al. 2005).

The simulation model for this example is a sector model extracted from the full field model. It is a 3-phase black-oil model descretized into 58x36x17 mesh or a total of 35,496 grid cells. The reservoir is under peripheral water injection. Initially, the model has 22 producers and 11 injectors as shown in Figure 3.11. The porosity and permeability fields for different layers are shown in Figure 3.12. The average porosity and permeability for layers deteriorate with depth as depicted by Figure 3.13. 


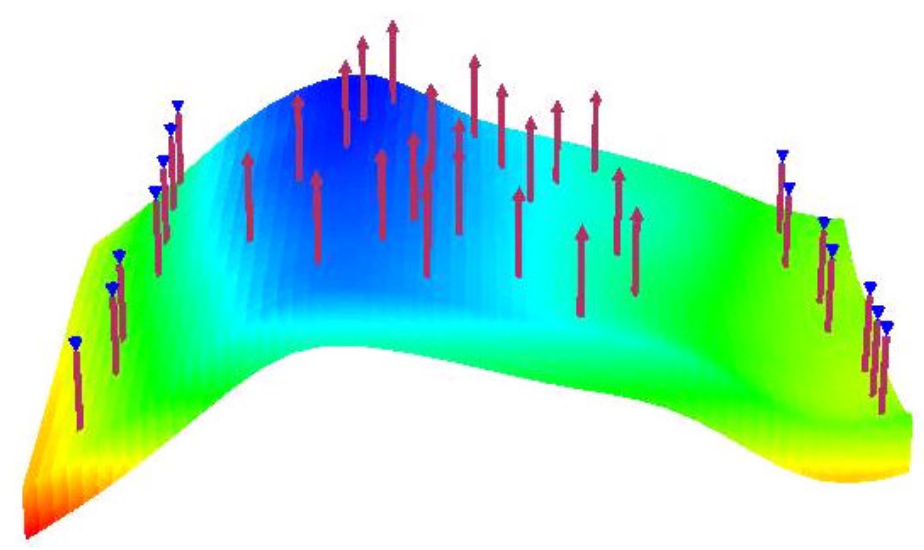

Figure 3.11 Well locations: 3D synthetic field case

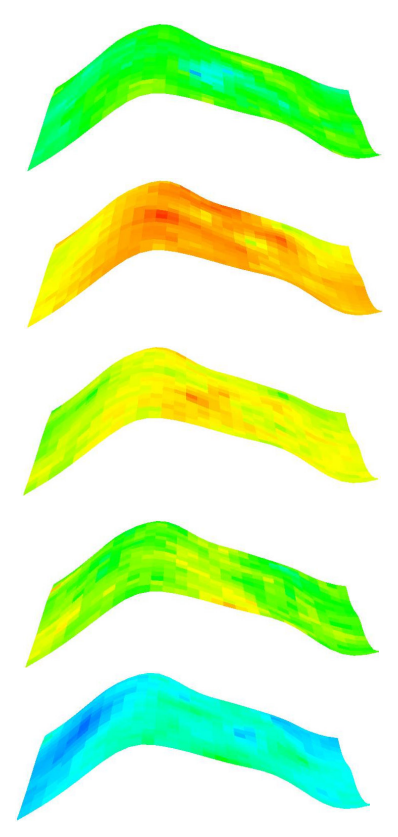

Porosity

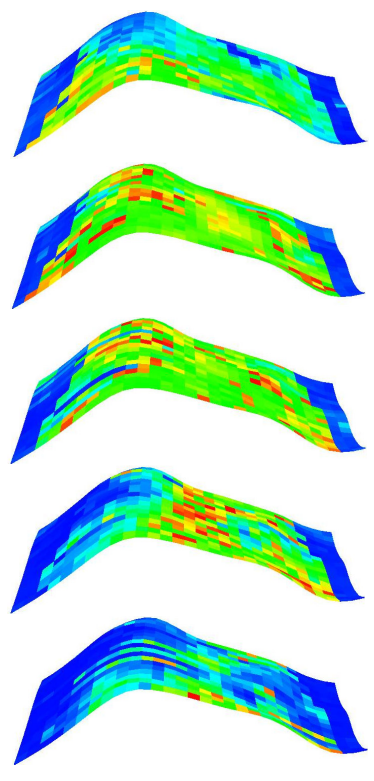

Permeability

Figure 3.12 Porosity and permeability fields for different layers: 3D synthetic field case 


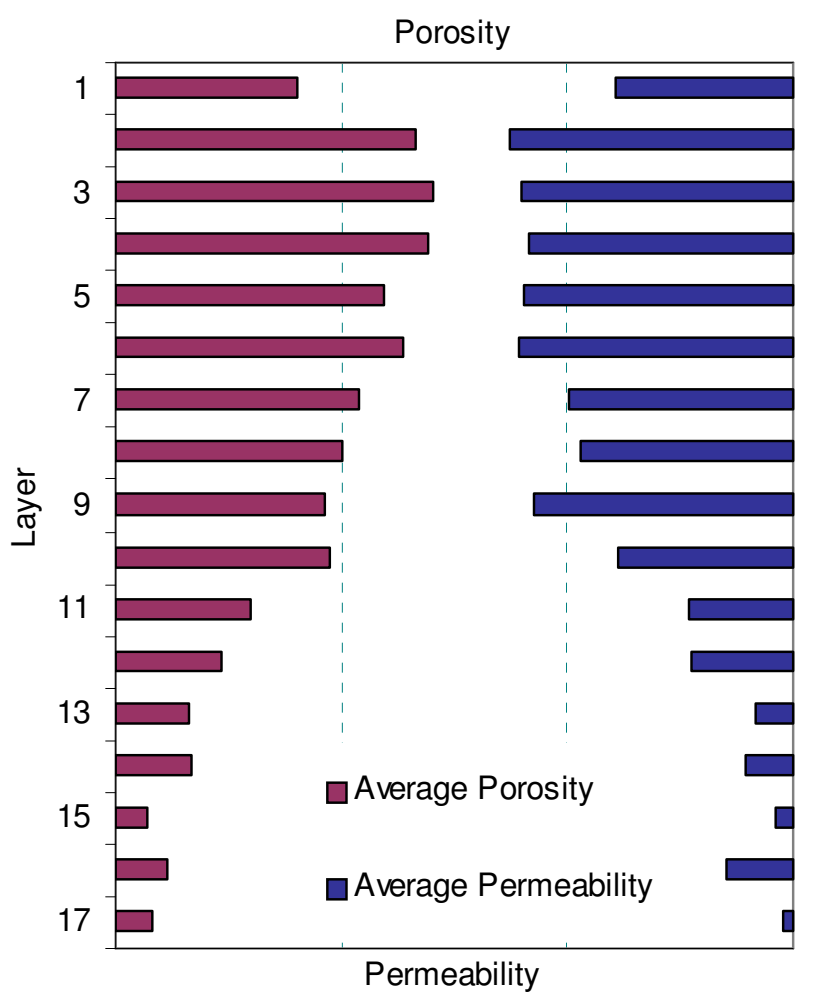

Figure 3.13 Average porosity and permeability per layers: 3D synthetic field case

It has been reported in the literature (Cosentino et. al. 2002) that part of this reservoir is fractured. These fractures have mainly a north-west trend as shown in Figure 3.14. We generated multiple realizations of discrete fracture networks (DFN) using prespecified distributions that control fracture length, height, and azimuth inside elliptical fracture swarms (Al-Harbi et al. 2005). The DFN patterns are then converted to a gridbased fracture permeability using a moving window to calculate the fracture density for each grid cell. The fracture density is used to enhance the matrix permeability and generate multiple single-permeability realizations for the synthetic field. Figure 3.15 shows a sample of the DFN realizations and the corresponding grid-based permeability distribution. 


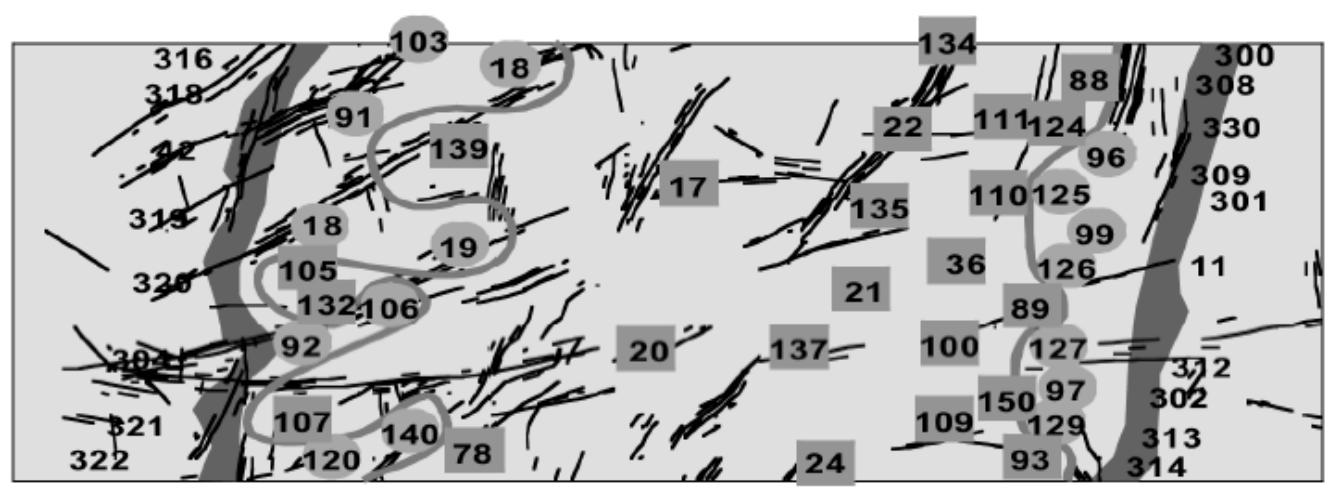

Figure 3.14 Integrated map showing mainly fracture distribution and orientations after Cosentino et al. (2002)
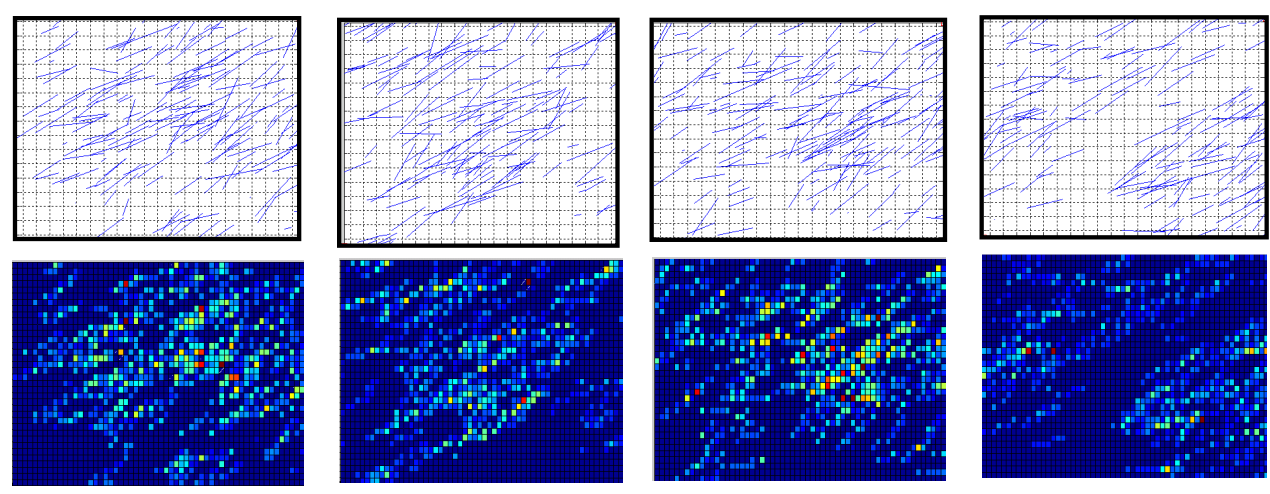

Figure 3.15 Discrete fracture network maps on the top and the corresponding grid-based fracture permeability maps below: 3D synthetic field case

The optimization is performed using 10 permeability realizations and $r=0$, that is risk-neutral conditions. It is implemented after 15 years of production when a smartcomplex well is added, making a total of 23 producers. The smart-complex well location and completion is shown in Figure 3.16. The well is completed horizontally towards the top of the reservoir, specifically the second layer. It consists of a mother-bore and two laterals with a total reservoir contact of about $4 \mathrm{~km}$. 


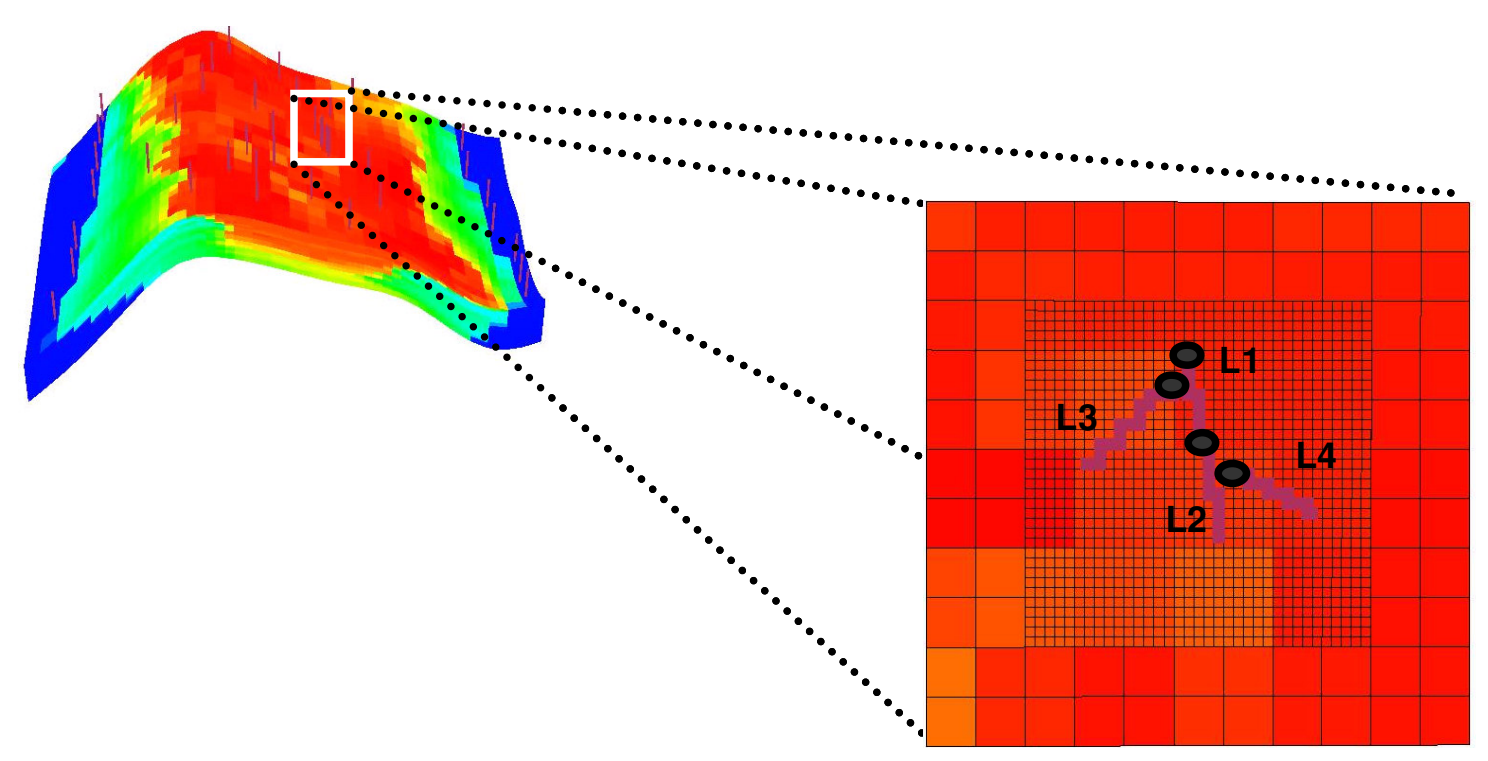

Figure 3.16 Smart-complex well completions, 2 ICVs along the mother-bore (L1 and L2) and one ICV at the entry of each lateral (L3 and L4): 3D synthetic field case

The well is equipped with four ICVs, two along the mother-bore and one at the entry of each lateral. For the purpose of the optimization, we will consider the complex well as four horizontal wells. The rate optimization will be performed with multiple equality and inequality constraints summarized as follows,

- Voidage replacements $\&$ total production $=400 \mathrm{M} R B / D$

- Maximum well total production $=35 \mathrm{M}$ RB/D

- Maximum well total injection $=70 \mathrm{MRB} / \mathrm{D}$

- Minimum well total production/injection $=1000 \mathrm{RB} / \mathrm{D}$

- Maximum complex well total production $<50 \mathrm{M}$ RB/D 


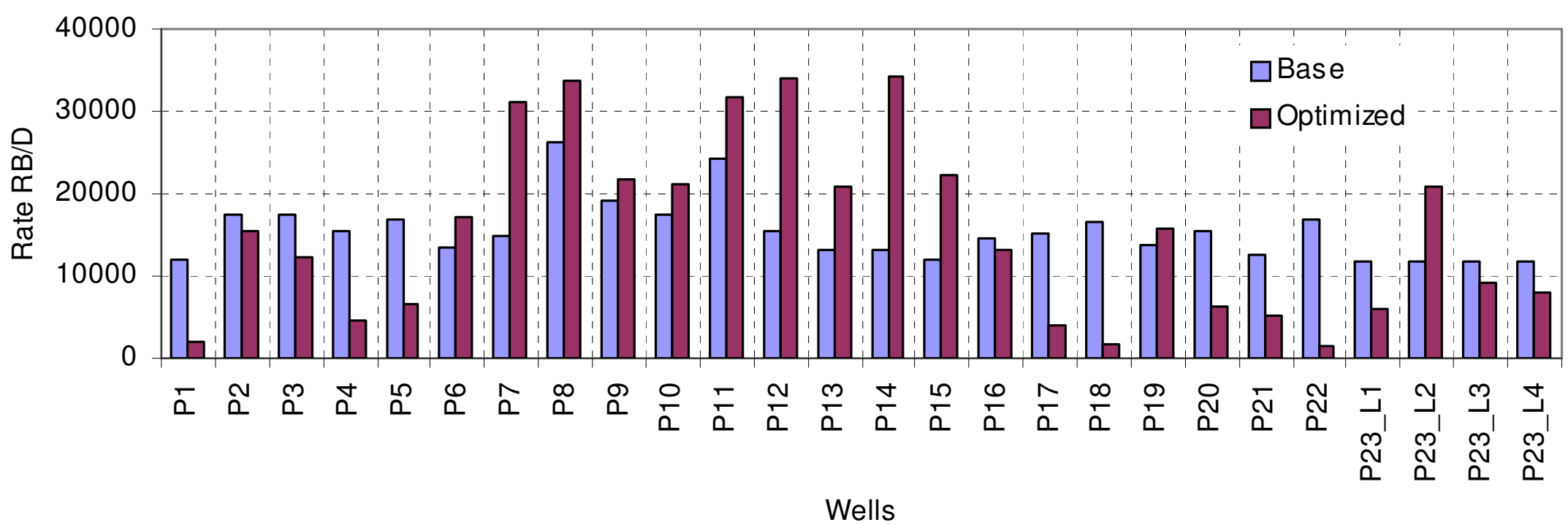

Figure 3.17 Comparison between the base case and the optimized case in terms of the total fluid production: 3D synthetic field case 


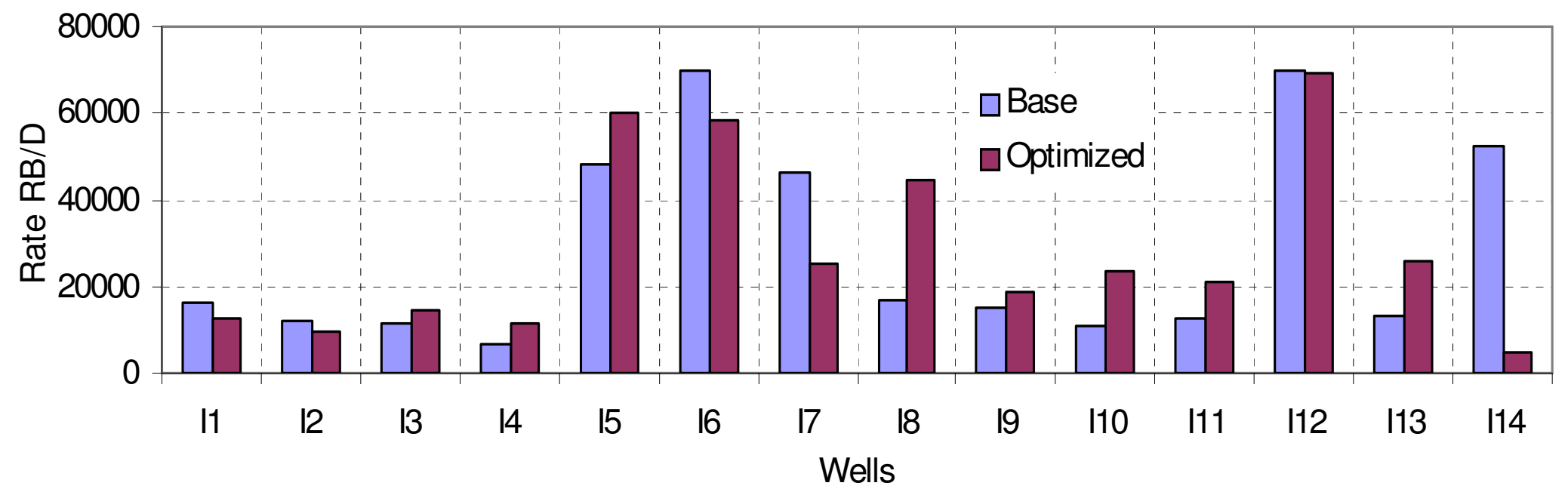

Figure 3.18 Comparison between the base case and the optimized case in terms of the total fluid injection: 3D synthetic field case 
The base case is chosen such that the production and injection rate are assigned based on the well productivity or injectivity indices. The complex well is assigned a production rate of $48 \mathrm{M} \mathrm{RB/D}$ as a base case, and the total rate is distributed equally among the four branches. Figures 3.17 and 3.18 compare the base case rates and the optimized case rates. The producer numbered 23 (P23) represents the smart-complex well.

The spatial distributions of rate allocation before and after optimization are shown in Figure 3.19 and 3.20 respectively. In terms of injection allocation, there has been no significant change before and after optimization on the western flank. However on the eastern flank, some of the injection rate was allocated from the southern injectors to the northern ones. In term of production allocations, there has been a significant change in the production rate allocation after optimization. Recall that the objective of the optimization is to equalize the water arrival time at all producers. For peripheral water injection in a homogeneous reservoir, intuitively we expect the production rates to increase towards the center of the field with increasing distance from the injectors. As we see, that is not necessarily the case for this strongly heterogeneous case. The complex well is produced at a total production of $43 \mathrm{M} \mathrm{RB/D}$ which is lower than the base case rate that was assigned.

The optimized case resulted in a reduction of the objective function by almost $60 \%$ from the base case where rates were allocated based on well productivity indices. Figure 3.21 shows the decrease in the objective function with iterations. 


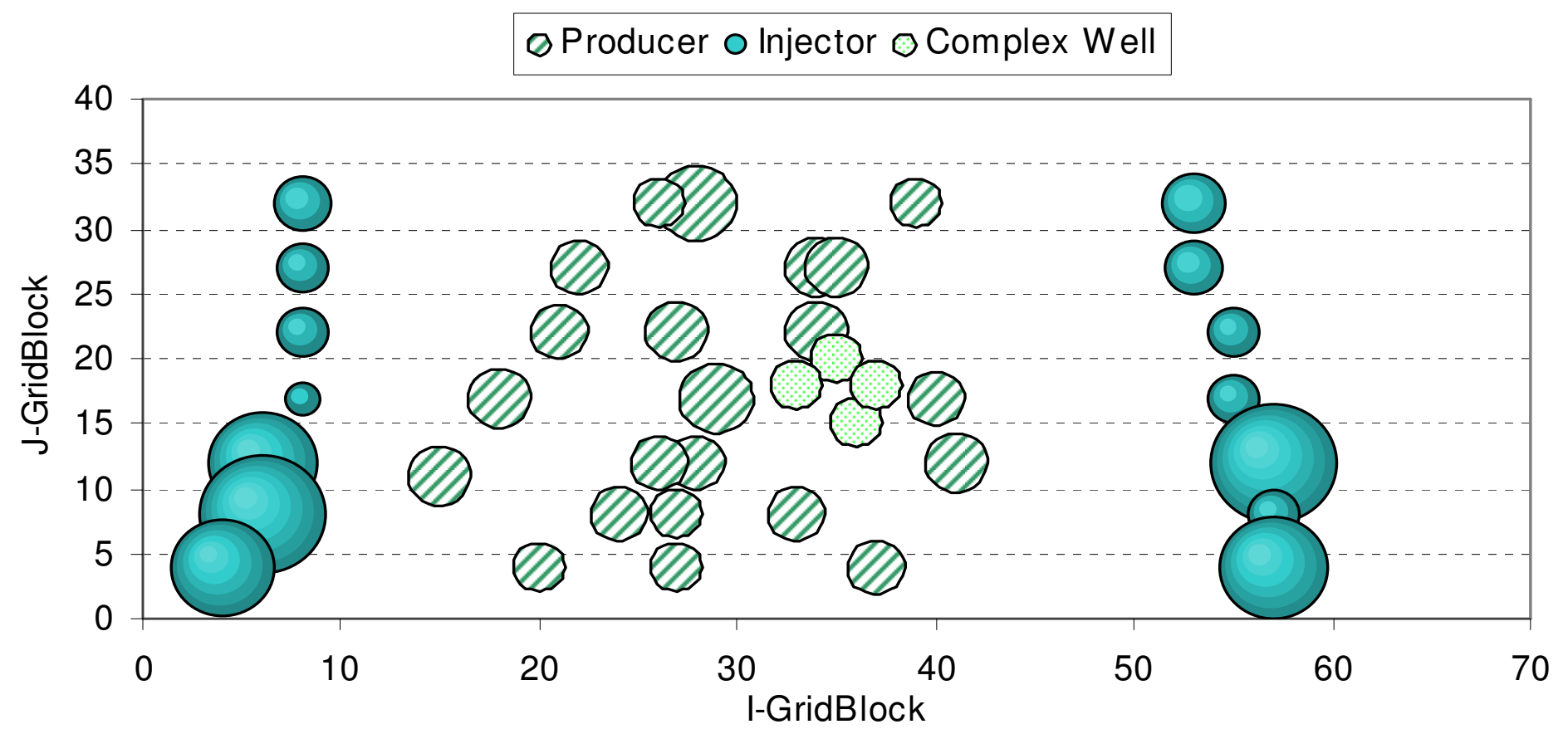

Figure3. 19 Bubble map showing rate allocations for the base case, the size of the bubble represents the amount of total injection/production rate: 3D synthetic field case 


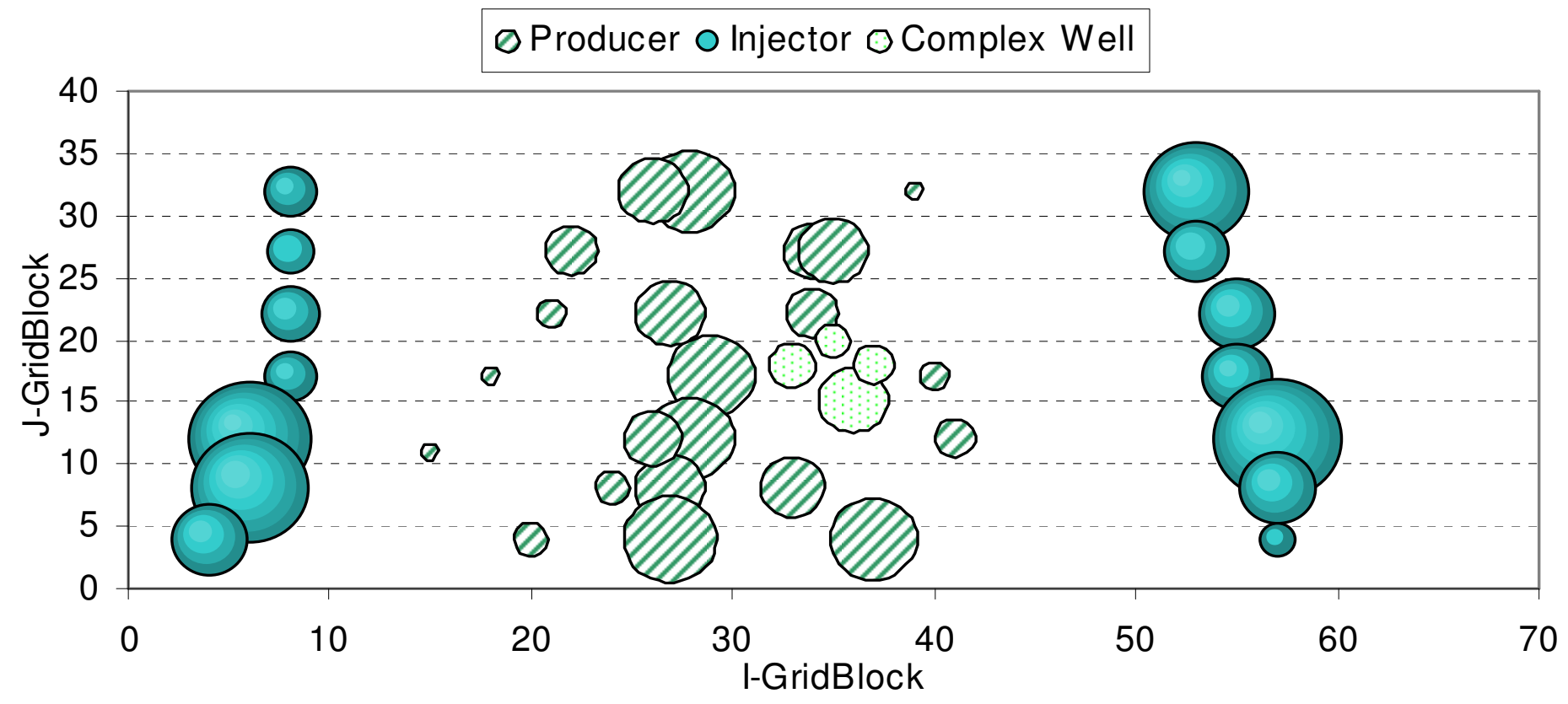

Figure 3.20 Bubble map showing fate allocations for the optimized case, the size of the bubble represents the amount of total injection/production rate: 3D synthetic field case 


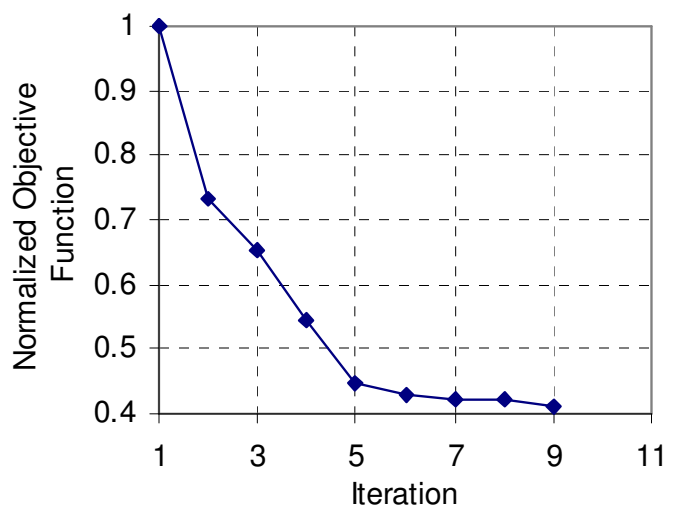

Figure 3.21 Normalized objective function evaluation per iteration: 3D synthetic field case

To examine the impact of optimization, we generated a new permeability distribution with the same geostatistical and fracture parameters and run the forward simulator for this model using the base case rate and the optimized rates for almost 2000 days. Figure 3.22 compares the water cut before and after optimization. There has been a significant reduction in the water cut, especially after the optimization was implemented.

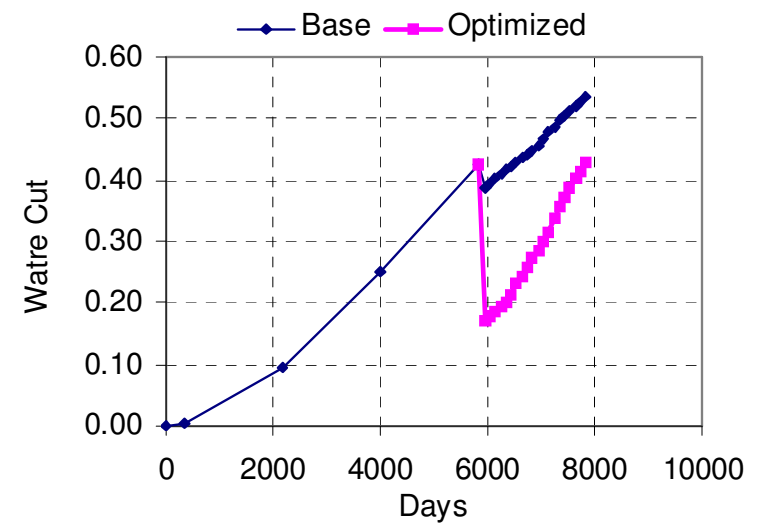

Figure 3.22 Comparison between the base case and the optimized case in terms of water cut: 3D synthetic field case 
To demonstrate the impact on the oil recovery, Figs. 3.23 and 3.24 show the cumulative oil and water production ratio after the optimization is implemented. These ratios are computed as follows,

$$
\text { Ratio }=\frac{\text { Cum. production from start of optimization }(\text { at } 15 \text { years })}{\text { Total Cum. production }}
$$

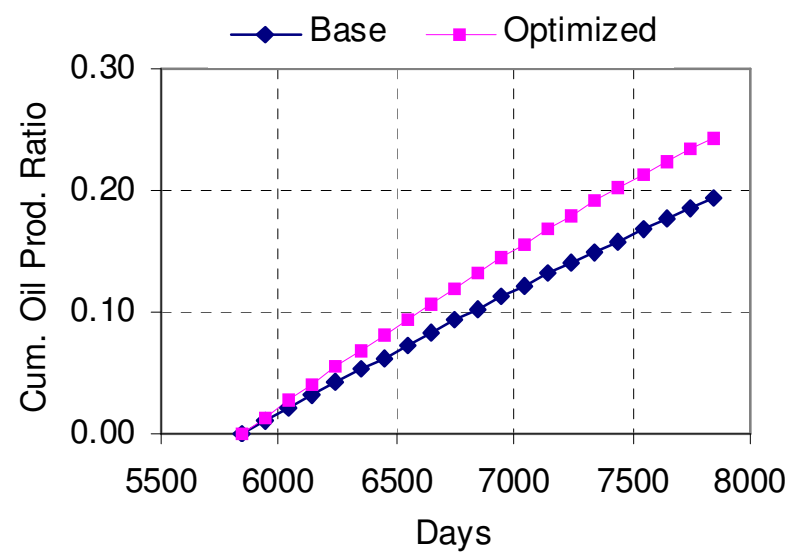

Figure 3.23 Cumulative oil production ratio vs. time: 3D synthetic field case

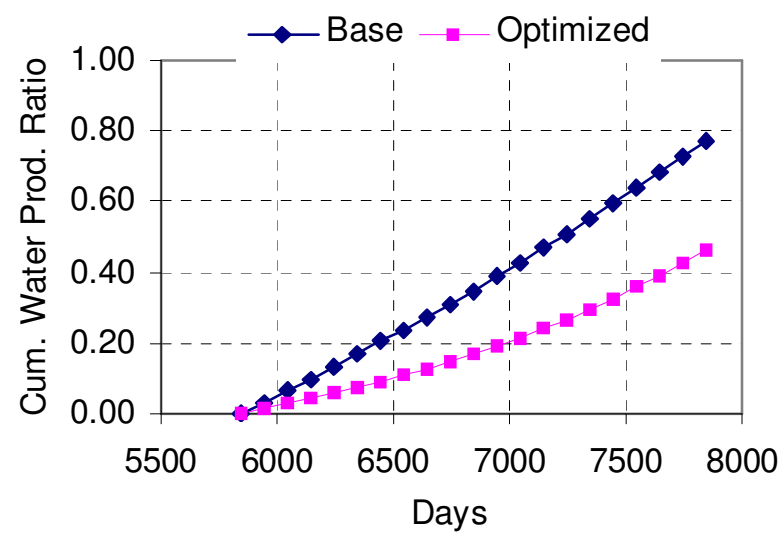

Figure 3.24 Cumulative water production ratio vs. time: 3D synthetic field case 
The numerator in Eq. 3.30 represents the cumulative production starting at 15 years when the optimization implemented. The denominator is the cumulative production from the beginning. An incremental cumulative oil production of $5 \%$ is realized after using the optimized rates. In addition, there has been a significant reduction in the cumulative water production (about 30\%).

\subsubsection{Measurements-Based Optimization (MBO): 2D Heterogeneous Case}

This section illustrates our approach of incorporating observed measurements in the optimization process. For this example, we show how to incorporate the 4D seismic derived saturation data if our objective is to minimize Eq. 3.2 with $r=$ zero. The same approach will apply for any other data, including production data. We use the previous 2D example shown in Figure 3.2. We assume a true model with the permeability field given in Figure 3.25. We run the forward simulator using this model for 200 days to generate a water saturation map which we will treat as measured data derived from 4D seismic surveys (Figure 3.26). We assumed a 10\% error in the $4 \mathrm{D}$ seismic data. The rates used for this simulation run are the base case rates. Next, we run the flow simulator for 32 realizations using the base case rates and generate water saturation maps for each realization after 200 days.

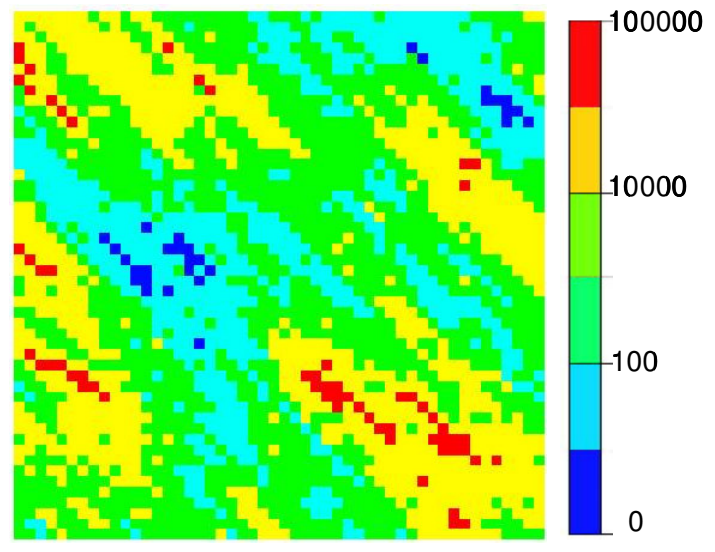

Figure 3.25 True permeability field: MBO 


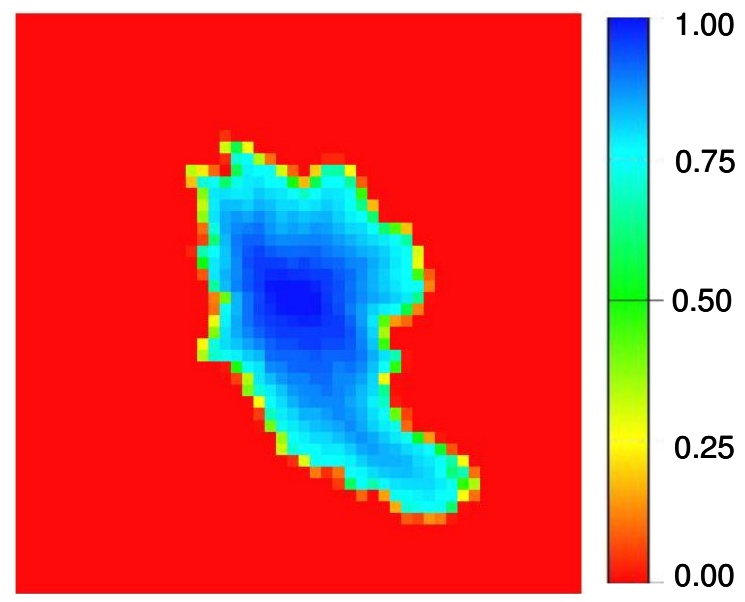

Figure 3.26 Water saturation map for the true model after 200 Days: MBO

We then computed the weights for each realization using Eq. 3.29. Figure 3.27 shows the weights obtained for each realization. It is clear that four realizations will dominate the optimization results in this case.

The optimization was performed after producing the field for 200 days. Figure 3.28 shows a comparison between the rates obtained using equal weights for 32 realizations and the rates obtained using the MBO. Figure 3.29 shows a comparison in terms of water cut versus oil recovery between the base case, the optimized case with equal weights, and the MBO case. These plots were generated using the true model and the optimized rates were used after 200 days of production. It is clear that using the optimized rates resulted in delaying the water breakthrough significantly. The MBO improved the results and delayed the water breakthrough even further. 


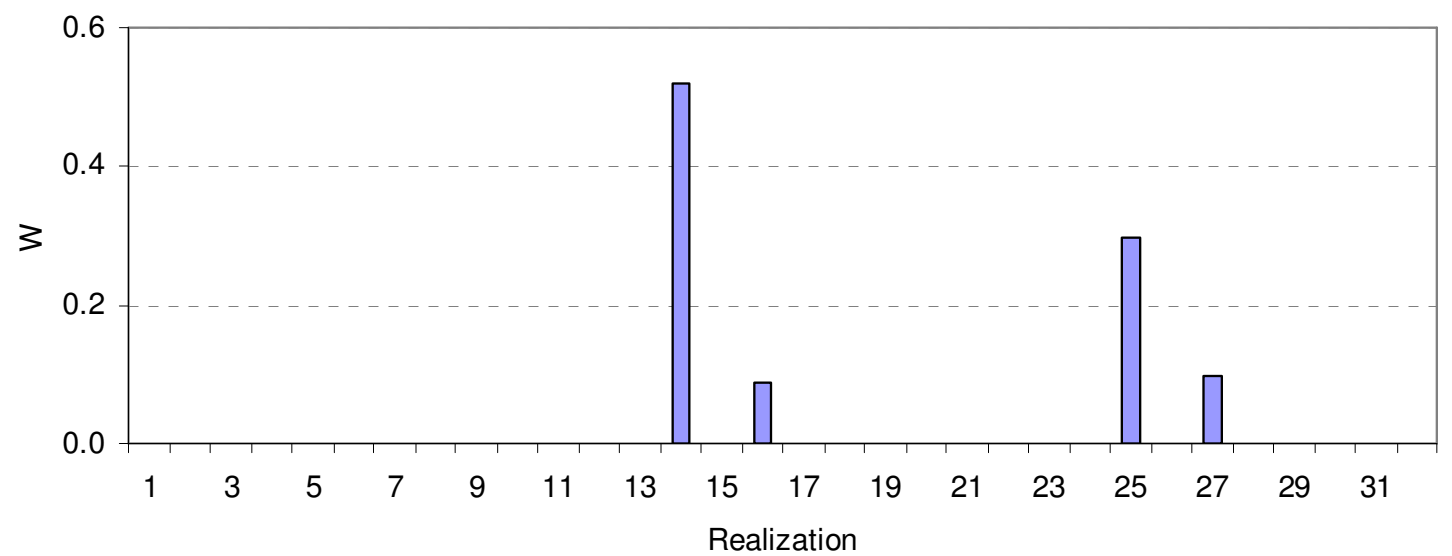

Figure 3.27 Calculated weight for 32 realizations using Bayes' Theorem: MBO

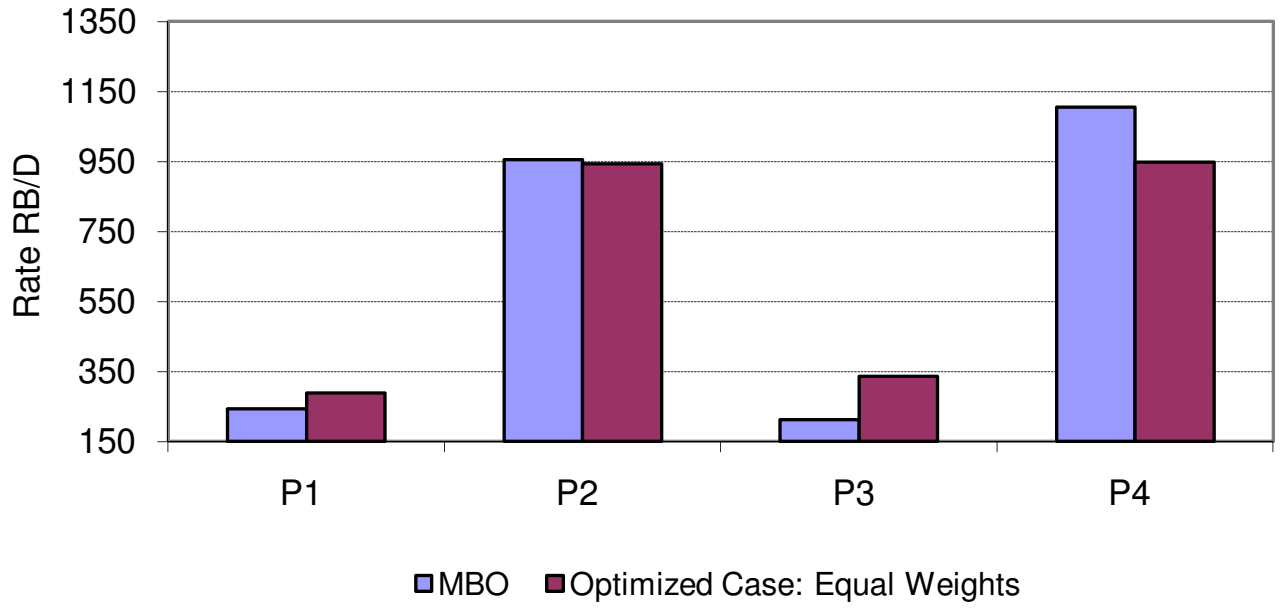

Figure 3.28 Comparison between the MBO and optimize case using equal weights in terms of total production rate: $\mathrm{MBO}$ 


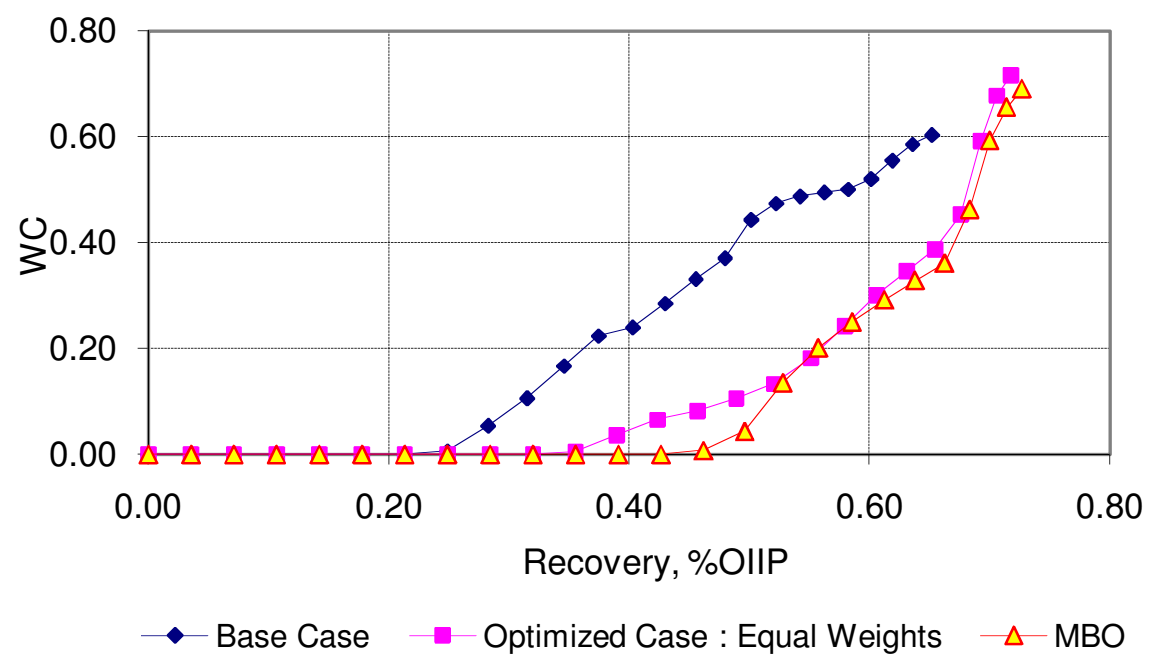

Figure 3.29 Comparison between the MBO, optimize case using equal weights, and the base case in terms of water cut vs. oil recovery: MBO

\subsection{Chapter Summary}

In this paper we proposed a practical and an efficient technique to optimize injection/production rates and maximize the sweep efficiency during waterflood accounting for geologic uncertainty. The approach is computationally efficient and shown to yield robust rates that can be implemented in field operations. Some specific conclusions from this study are summarized as follows:

- Geologic uncertainty was addressed in terms of two forms of objective functions:

(i) a stochastic form which includes the expected value and the standard deviation combined with a risk coefficient and (ii) a min-max form which minimizes the worst case scenario.

- Analytical formulations of the objective function gradients and Hessian were derived. These formulations require only one flow simulation per realization regardless of the number of injectors and producers and leads to an efficient optimization algorithm. 
- The proposed measurement-based optimization provides a simple mechanism to incorporate production history during optimization.

- The power and practical feasibility of our method has been demonstrated by a synthetic field example based on a giant middle-eastern field. We optimized the production rates for 36 wells including a smart-complex well. The optimization was performed while accounting for geologic uncertainty using 10 realizations.

- In all studied cases, our approach improved sweep efficiency and increased oil production with a corresponding decrease in the water-cut response. 


\section{CHAPTER IV}

\section{APPLICATIONS OF WATERFLOOD OPTIMIZATION VIA OPTIMAL RATE CONTROL WITH SMART WELLS*}

In this chapter, we revisit the optimization algorithm developed in the previous chapter and demonstrate its practical feasibility through large-scale field applications. We also propose an efficient method to handle some of the nonlinear constraints that arise during a waterflood project. These constraints have a nonlinear relationship with respect to the control variables which are wells rates in our study. We impose two such constraints in our optimization viz. the minimum or maximum flowing bottom-hole pressure (FBHP) constraints and rate restrictions on high water cut wells. The need for those constraints generally arises from facility-related limitations or reservoir management guidelines. We have applied our method to two examples. The first one is a 3D synthetic field named Brugge field which is a benchmark case developed by TNO (Peters et al. 2009). The reservoir properties for the case are based on a North Sea Brent-type field. Ten years of historical production data was available for this synthetic case. The production data was used to history match 30 geologic realizations and updated models were used in the production optimization process. Out of the 30 historymatched models, ten realizations were used to conduct the optimization over 30 smart wells, each equipped with 3 ICVs. The rest of the realizations were used to test the optimized rates. The second case is a real field example from the middle-east with more than 300 wells. Most of the wells are vertical wells and their rates are controlled manually in the field using surface chokes on a monthly basis. In addition, the study area includes a few horizontal wells and a smart well. The smart well is a maximum reservoir

\footnotetext{
*Part of this chapter is reprinted with permission from "Field Applications of Waterflood Optimization via Optimal Rate Control with Smart Wells," Ahmed H. Alhuthali, Akhil Datta-Gupta, Bevan Yuen, and Jerry P. Fontanilla, paper SPE 118948 presented at the 2009 SPE Reservoir Simulation Symposium, The Woodlands, Texas, February 2-4, Copyright 2009 by the Society of Petroleum Engineers.
} 
contact (MRC) well with horizontal section extending for around $5 \mathrm{~km}$ and equipped with 4 ICVs. Our optimization results demonstrate the viability of our approach with a substantial increase in the cumulative oil production and a considerable decrease in the associated water production.

\subsection{Handling Non-linear Constraints}

In this section we briefly discuss our approach to handle some of the nonlinear constraints that arise during field applications, specifically, the minimum or maximum allowable flowing bottom-hole pressure and rate restriction on high water cut wells.

\subsubsection{The Minimum Allowable Flowing Bottom-Hole Pressure (FBHHP) (Pwmin)}

In waterflood projects, field operators may impose a restriction on the minimum allowable flowing bottom-hole pressure based on operation or reservoir management concerns. Our optimization method uses wells rates as control variables and not the FBHP. Thus, the $P_{w f \text { min }}$ needs to be added as a constraint during the optimization process. Mathematically, it can be treated as an inequality constraint as follows,

$$
P_{w f, i} \geq P_{w f, \text { min }}
$$

Equation 4.1 dictates that the FBHP for well $i, P_{w f, i}$, should be greater or equal to $P_{w f, \text { min }}$ at all times. The relationship between $P_{w f, i}$ and the control variables, rates, has the following general form,

$$
q_{i}=P I_{i}\left(P_{r, i}-P_{w f, i}\right)
$$

The variables $P I_{i}$ and $P_{r, i}$ in Eq. 4.2 represents the well productivity index for well $i$ and reservoir pressure respectively. This relationship is non-linear because the reservoir pressure changes with time. However, we assume steady state conditions within the time interval of optimization. This is a reasonable approximation for waterflood as the total 
mobility is a relatively weak function of saturation. The inequality in Eq. $\mathbf{4 . 1}$ can be written in terms of rates as follows,

$$
q_{i}^{k} \leq q_{i, \max }^{k}
$$

The superscript $k$ represents the iteration number. Normally, our optimization will be implemented over multiple optimization time intervals and each optimization interval will consist of multiple iterations to minimize the objective function. The variable $q_{i, \max }$ represents the maximum allowable rate given by,

$$
q_{i, \max }^{k}=P I_{i}^{k}\left(P_{r, i}^{k}-P_{w f, \min }\right)
$$

We approximate $q_{i, \max }$ explicitly using the information from the previous iteration, $k-1$.

$$
q_{i, \max }^{k} \approx q_{i}^{k-1} \frac{\left(P_{r}^{k-1}-P_{w, \min }\right)}{\left(P_{r}^{k-1}-P_{w, i}^{k-1}\right)}
$$

In Eq. 4.5, we have used the production rate allocated during the previous iteration along with the reservoir and flowing bottom hole pressure. The approximation in Eq. 4.5 relies on the assumption that the reservoir pressure will not change substantially between successive iterations. Figure 4.1 shows cross plots of normalized well block pressures for consecutive iterations for several wells for a real field case that we will discuss later. These results show that the well block pressures, indeed, do not change between successive iterations which is consistent with our assumptions. This is also because of the fact that the rate changes between successive iterations are also not very large as shown in Figure 4.2. 

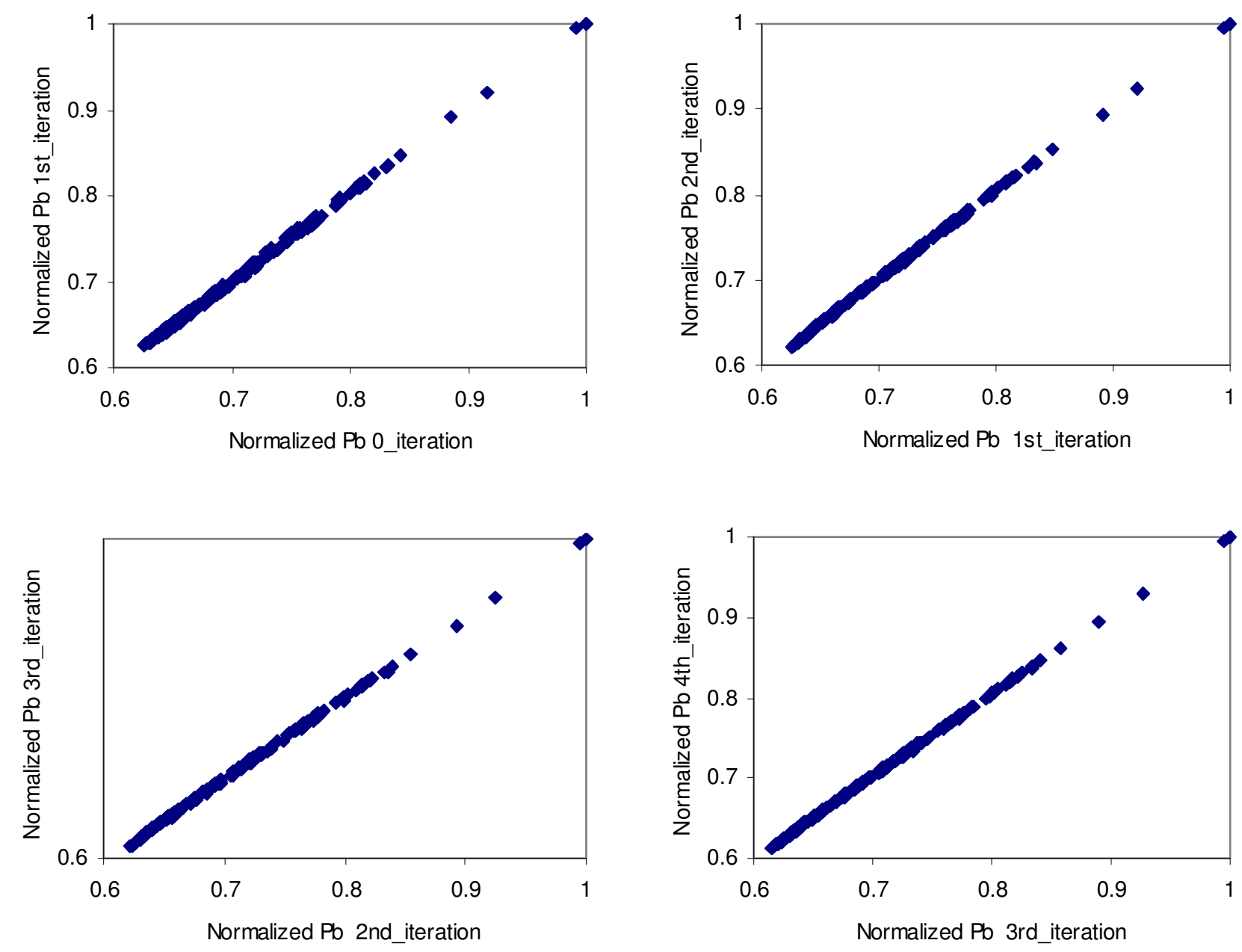

Figure 4.1 Cross plot to compare wells block pressures between multiple iterations within a single optimization time step 

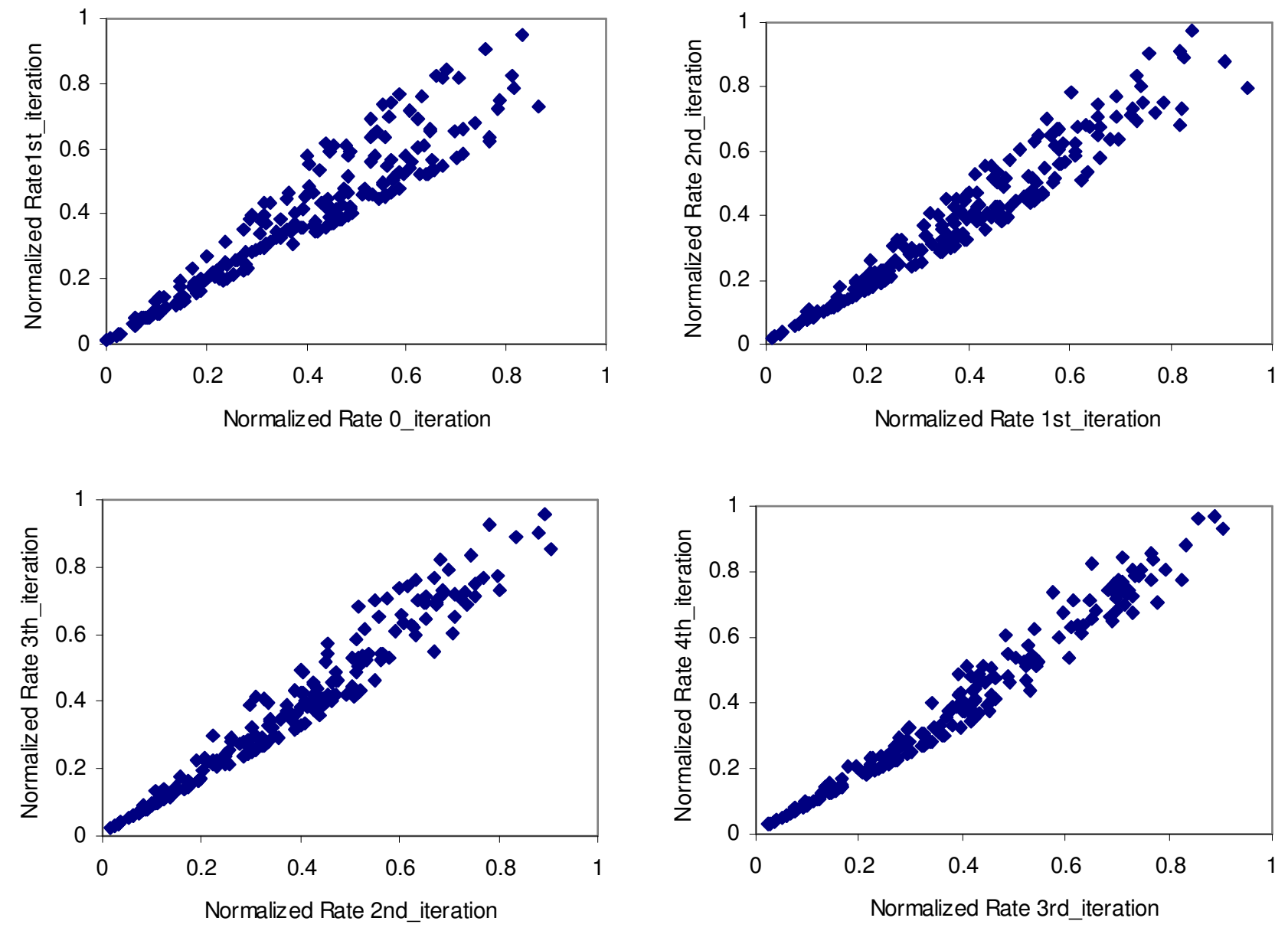

Figure 4.2 Cross plot to compare well rates between multiple iterations within a single optimization time step 


\subsubsection{Rate Restriction on High Water Cut Well.}

In this section, we briefly discuss how we impose restrictions on high water cut Hwells by modifying the bound constraints. Suppose we would like to cap the production of well $i$ to a specific total production $q_{w c r}$ if the well water cut, $w c_{i}$, exceeds some preset limit $w c_{r}$. This can be mathematically written as follows,

$$
q_{i} \leq q_{w c_{r}} \text { if } w c_{i} \geq w c_{r}
$$

The relationship between $q_{i}$ and $w c_{i}$ is not linear and implementing Eq. 4.6 requires additional assumptions. Specifically, we assume that if we are at $k-1$ iteration of a specific optimization time interval, then the $w c$ in well $i$ won't change substantially from iteration $k-1$ to $k$ because of a well rate reallocation. Figure 4.3 shows cross plots for water cut normalized to the highest water cut from almost 300 wells between successive iterations for a real field example to be discussed later. The results seem to validate our assumptions. It is important to point out that to ensure efficient handling of the nonlinear constraints, the optimization is carried out over multiple optimization time intervals. 

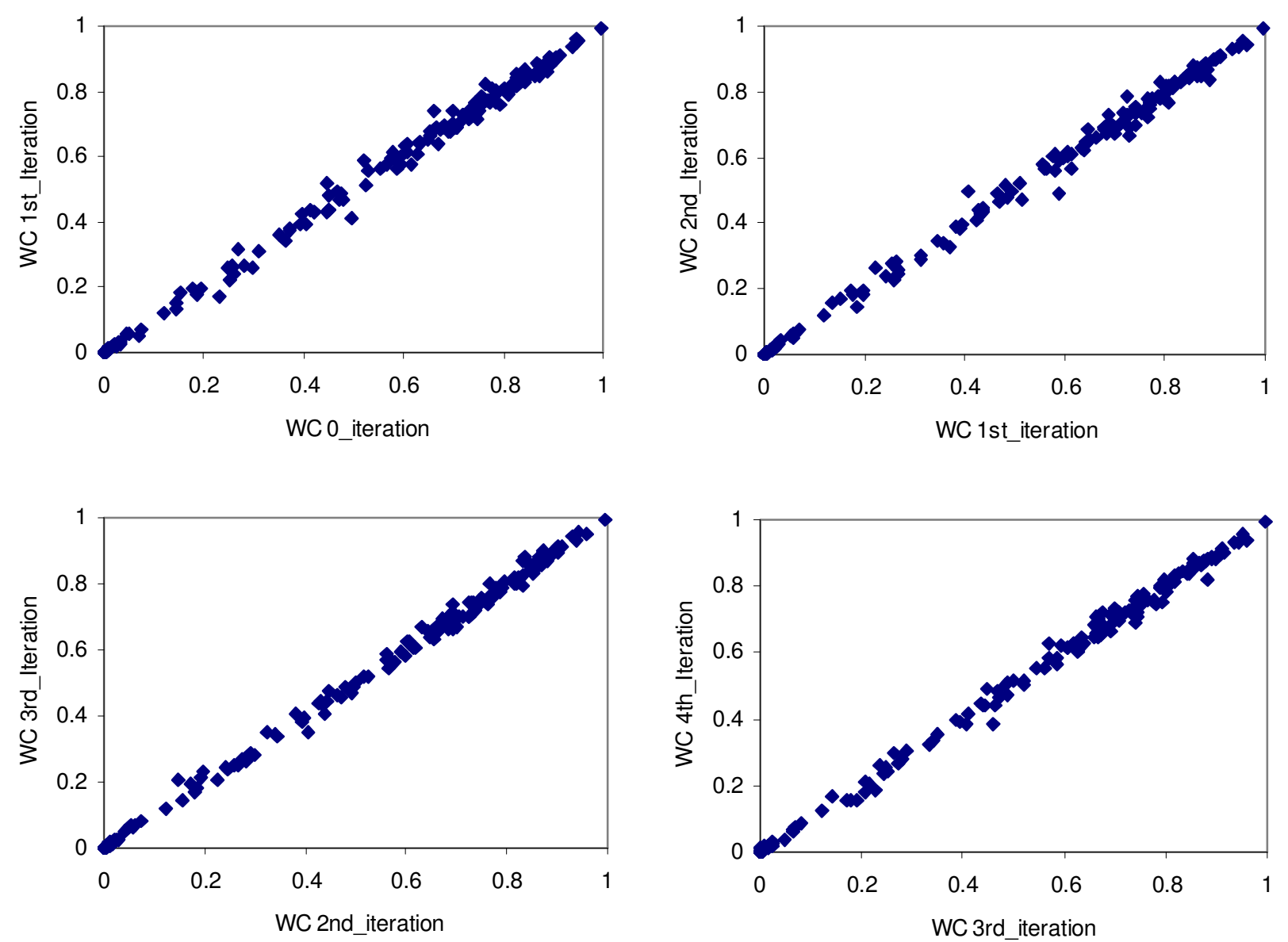

Figure 4.3 Cross plot to compare well water cut between multiple iterations within a single optimization time step: the water cut is normalized to the highest water cut 


\subsection{Applications and Discussion}

One of the main objectives of our paper is to demonstrate the practical feasibility of our previously approach for optimal rate control using field examples. Here we discuss applications to two cases. The first example is a 3D synthetic benchmark example, called the 'Brugge' field. The second example is a real field application on a giant middle-eastern field.

\subsubsection{A Synthetic Field Example: Brugge Field}

In this section we will present a closed loop approach to optimize the production from the Brugge field over 20 years. The Brugge field is a synthetic benchmark case that was set up by TNO as part of an SPE ATW to evaluate various closed loop control strategies. The details for this case can be found in Peters et al. (2009).

Reservoir Models. A series of model realizations were generated based on a reservoir properties and well log attributes extracted from a highly-resolution model consisting of 20 million grid cells. The Brugge field properties are based on a North Sea Brent-type field. The field was divided into 4 zones and the average properties of these zones are summarized in Table 1. The structure of the Brugge field consists of an E-W elongated half-dome with a large boundary fault at its north edge, and one internal fault with a modest throw at an angle of around 20 degrees to the north boundary fault. The model consists of 60000 gridblocks with 9 layers. It has 20 vertical producers completed mainly in the top 8 layers and 10 peripheral injectors completed in all 9 layers. The field structure and well locations are shown in Figure 4.4. 


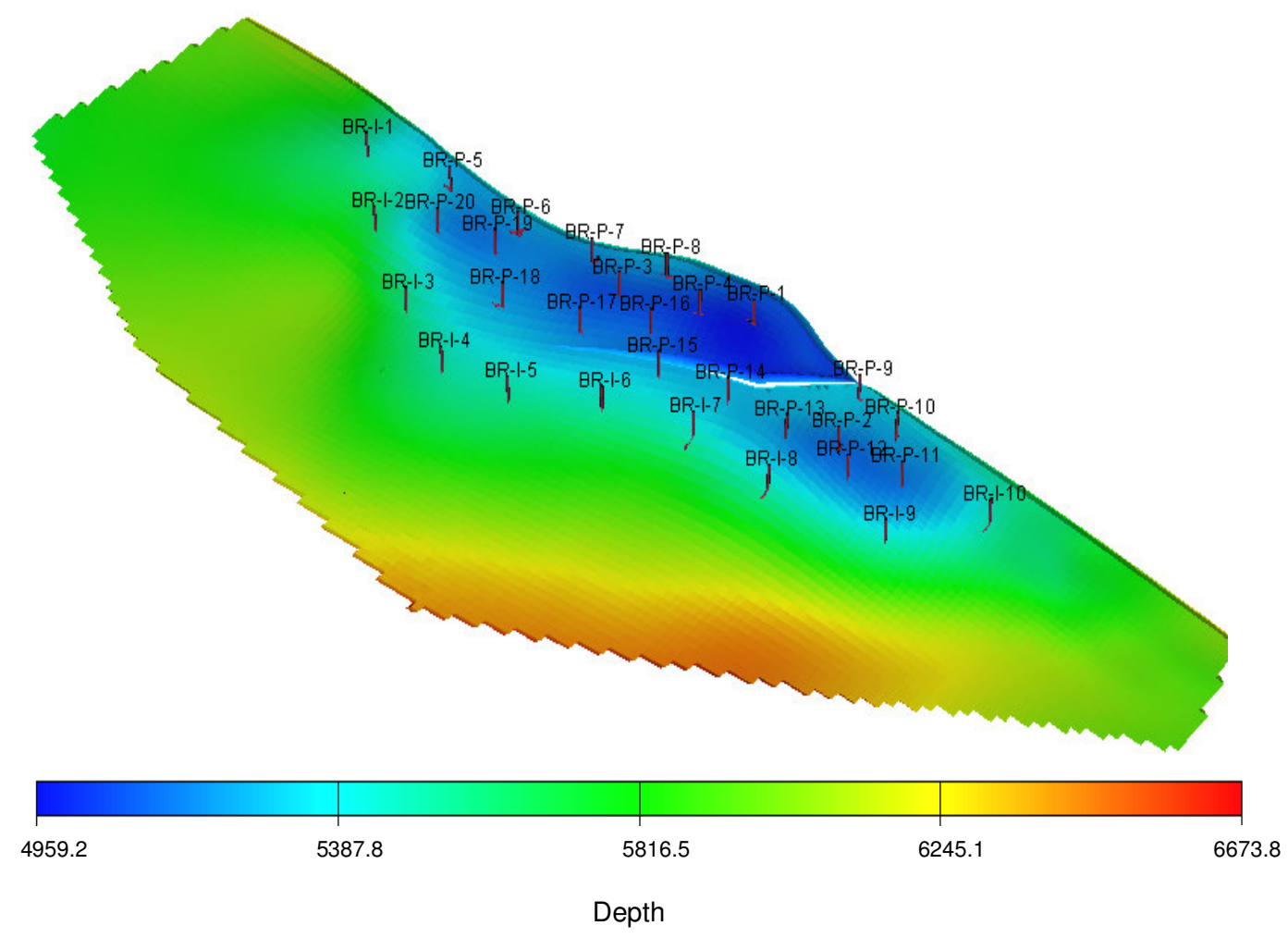

Figure 4.4 Map showing the structure of Brugge field and well locations

The first 10 years of the production history of the field was provided for history matching purposes. The production history was based on a 'true model' response with added noise. The closed loop control approach consisted of two steps: (i) model updating via production data integration using the field production history for the first 10 years and (ii) rate optimization whereby rates are allocated over the next 20 years.

Production Data Integration: For integration of water-cut response, we have used the streamline-based generalized travel time inversion (He et al. 2002). In this approach the water-cut data misfit is quantified in terms of an 'optimum' travel timeshift that maximizes the cross-correlation between the observed data and simulated response. Compared to traditional 'amplitude inversion', the travel time inversion has been shown to have favorable quasilinear properties that ensures rapid convergence 
(Cheng et al. 2005). We carry out matching of the flowing bottom-hole pressure data in the frequency domain following the procedure outlined by Vasco and Karasaki (2006). We take a Fourier transform of the bottom-hole pressure data and match only the zero-th frequency component of the data.

The data assimilation step involves minimization of a penalized misfit function consisting of the following three terms

$$
\|\delta \mathbf{d}-\mathbf{S} \delta \mathbf{R}\|+\beta_{1}\|\delta \mathbf{R}\|+\beta_{2}\|\mathbf{L} \delta \mathbf{R}\|
$$

In the above expression, $\delta \mathbf{d}$ is the vector of generalized travel time shift of water-cut and zero-frequency pressure misfit at the wells, and $\mathbf{S}$ is the stacked matrix of the sensitivity of generalized travel-time and the zero frequency pressure to reservoir parameters. These sensitivities are obtained semi-analytically during the flow simulation with minimal additional computational expense. Also, $\boldsymbol{d}$ correspond to the change in the reservoir property (for example, permeability) and $\mathbf{L}$ is a second spatial difference operator. The first term ensures that the difference between the observed and calculated production response is minimized. The second term, called a 'norm constraint', penalizes deviations from the prior model that already incorporates available geologic and static information related to the reservoir. Finally, the third term, a 'roughness penalty', simply recognizes the fact that production data is an integrated response and is thus, best suited to resolve large-scale structures rather than small-scale property variations.

The minimization is carried out via an iterative least-squares solution of the augmented linear system

$$
\left(\begin{array}{c}
\mathbf{S} \\
\beta_{1} \mathbf{I} \\
\beta_{2} \mathbf{L}
\end{array}\right) \delta \mathbf{R}=\left(\begin{array}{c}
\delta \mathbf{d} \\
\mathbf{0} \\
\mathbf{0}
\end{array}\right)
$$

The weights $\beta_{1}$ and $\beta_{2}$ determine the relative strengths of the prior model and the roughness term. An iterative sparse matrix solver, LSQR, is used for solving this 
augmented linear system efficiently. The LSQR algorithm is well suited for highly illconditioned systems and has been widely used for large-scale tomographic problems in seismology (Paige and Saunders 1982).

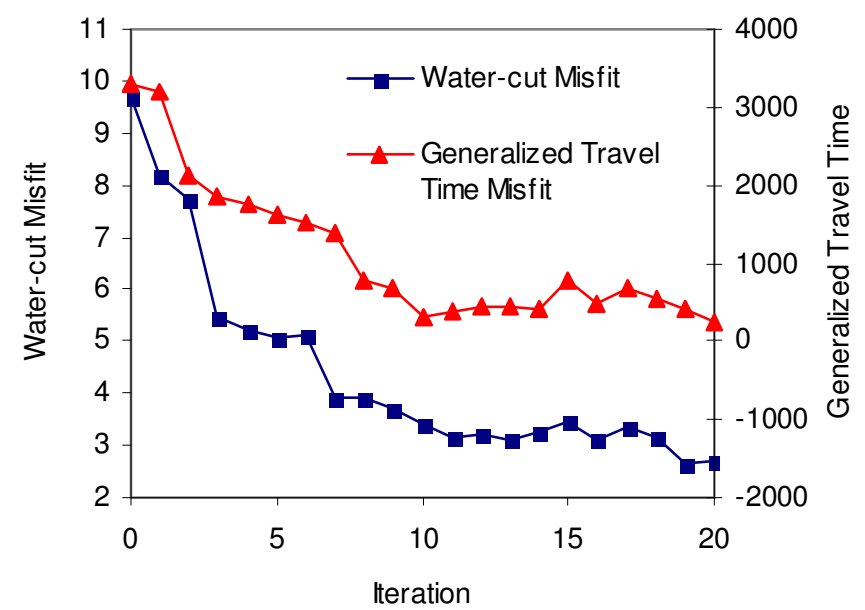

Figure 4.5 Water cut misfit and generalized travel time misfit

The history matching of the Brugge field was implemented by changing the permeability field ( $\mathrm{Kx}$ and $\mathrm{Ky}$ ). The history matching was performed for 30 realizations. Ten of these realizations are used later in the optimization process and the rest are used to validate the optimization results. Figure 4.5 shows the changes in water cut 'amplitude' misfit and the 'generalized travel time misfit' for one of the history matched realizations. The generalized travel time misfit is defined as the 'optimal time shift' that maximizes the cross-correlation between the observed and the calculated water cut. The water cut amplitude misfit is defined as the difference between the observed water cut and the calculated response from the reservoir simulator. This figure shows the decreases in the misfits as the iteration number increases. Figure 4.6 compares the initial permeability field and the history matched model for a few layers and also displays the changes from history matching. 


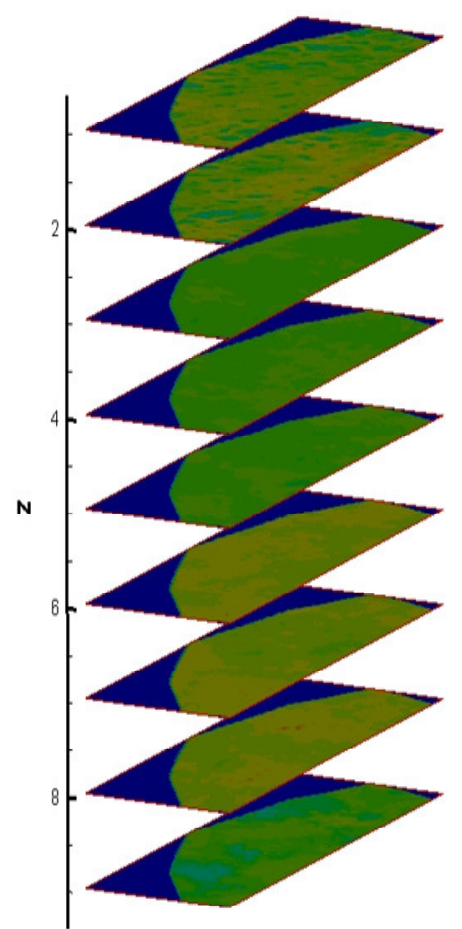

Initial

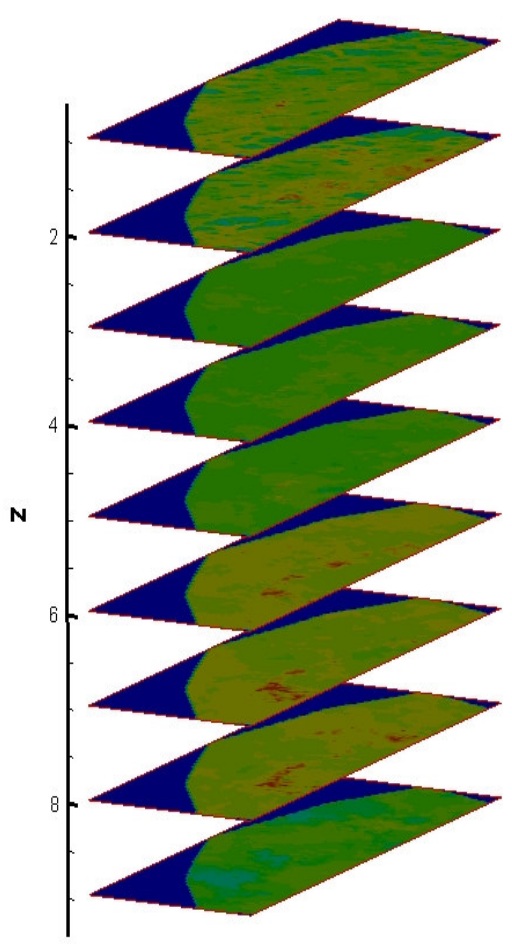

Match

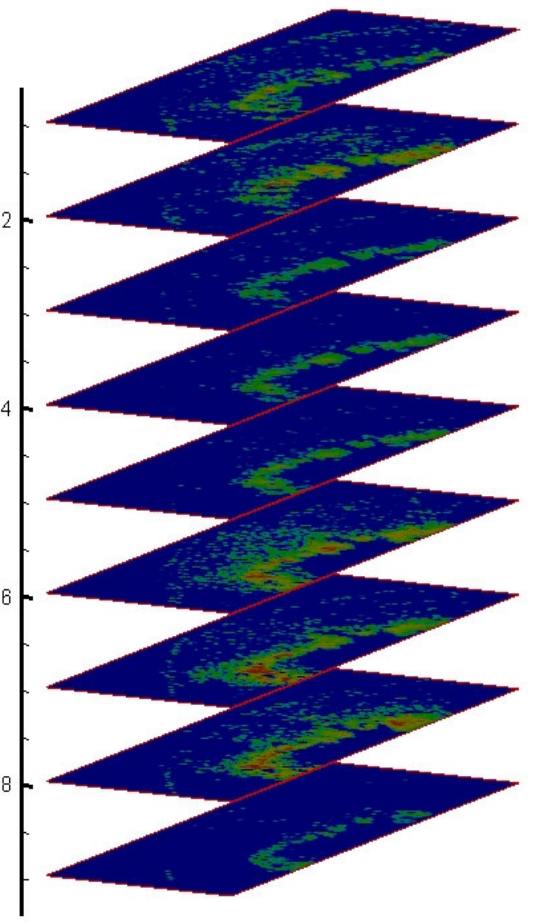

Difference

Figure 4.6 Permeability changes after history matching 
Significant improvement in the overall field history match was achieved in terms of oil and water production as shown in Figure 4.7.

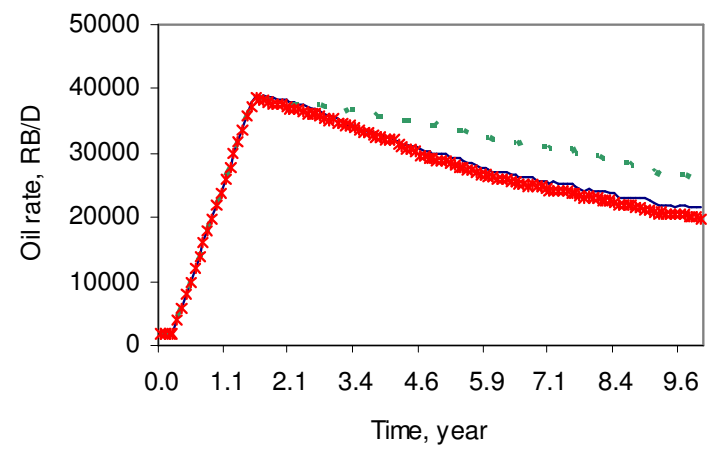

(a) Oil rate

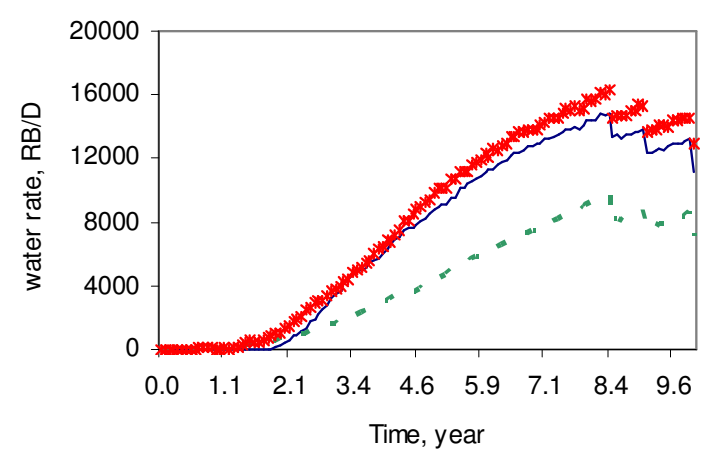

(b) Water rate

* Observed $=$ - - Initial $\longrightarrow$ Matched

Figure 4.7 History matching results for the field overall production: Brugge field

For individual wells, Figure 4.8 shows the quality of the water-cut history match for 8 producers. A satisfactory history match was achieved for all these wells in terms of water cut. Similarly for FBHP, a significant improvement was achieved for the 8 producers as shown in Figure 4.9. 
BR-P-2

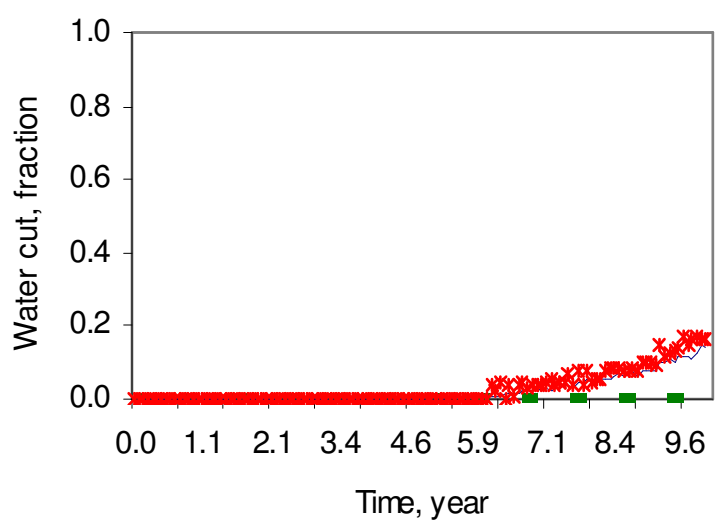

BR-P-6

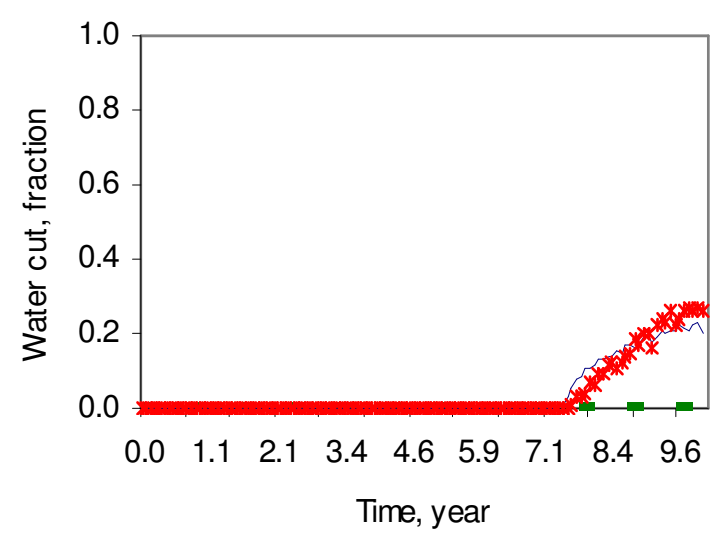

BR-P-5

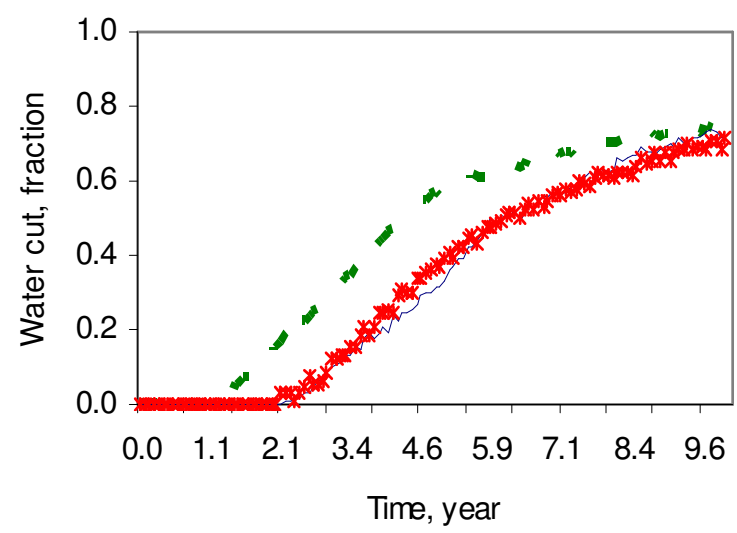

BR-P-10

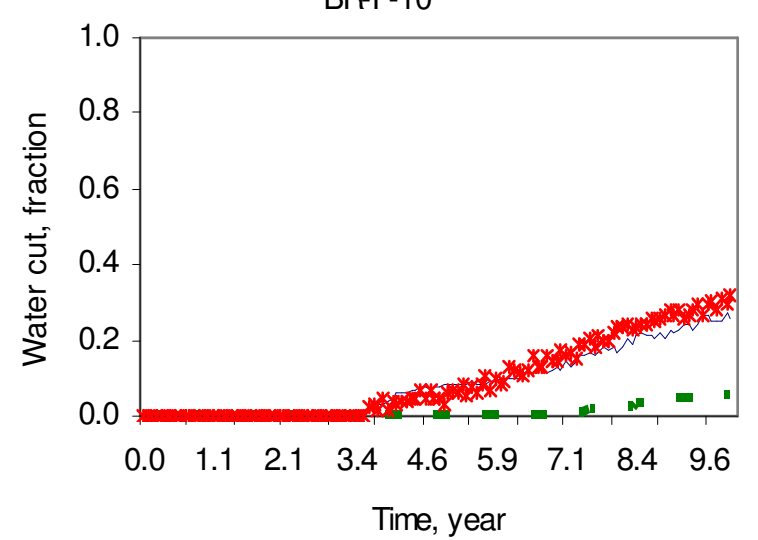

(a) Comparison for the first group of wells

Figure 4.8 Comparisons between observed, initial, and matched water cut for multiple wells: Brugge field 

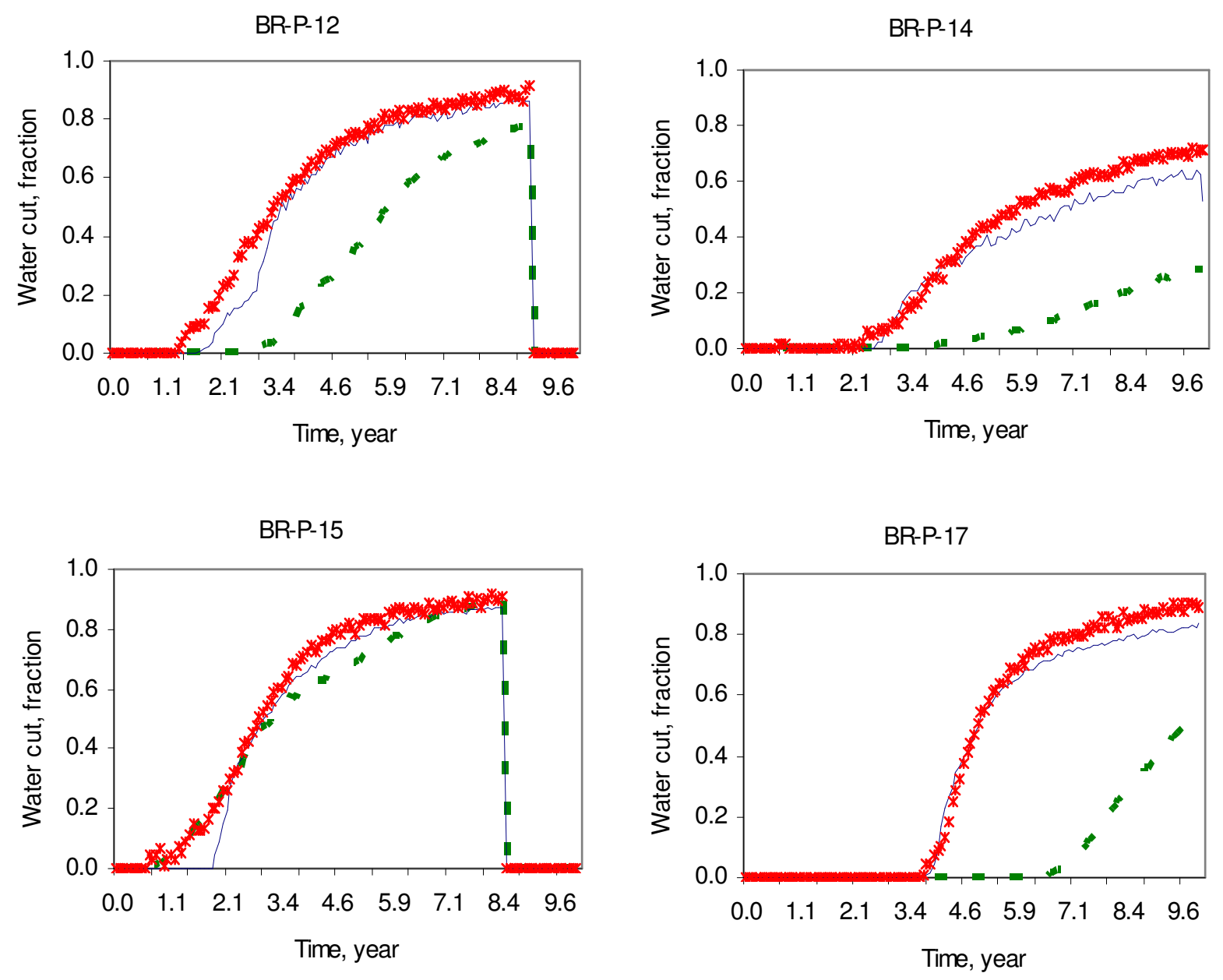

(b) Comparison for the second group of wells

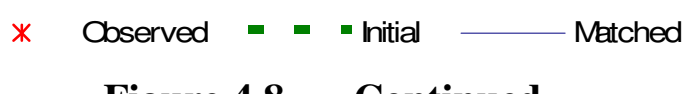

Figure 4.8 Continued 

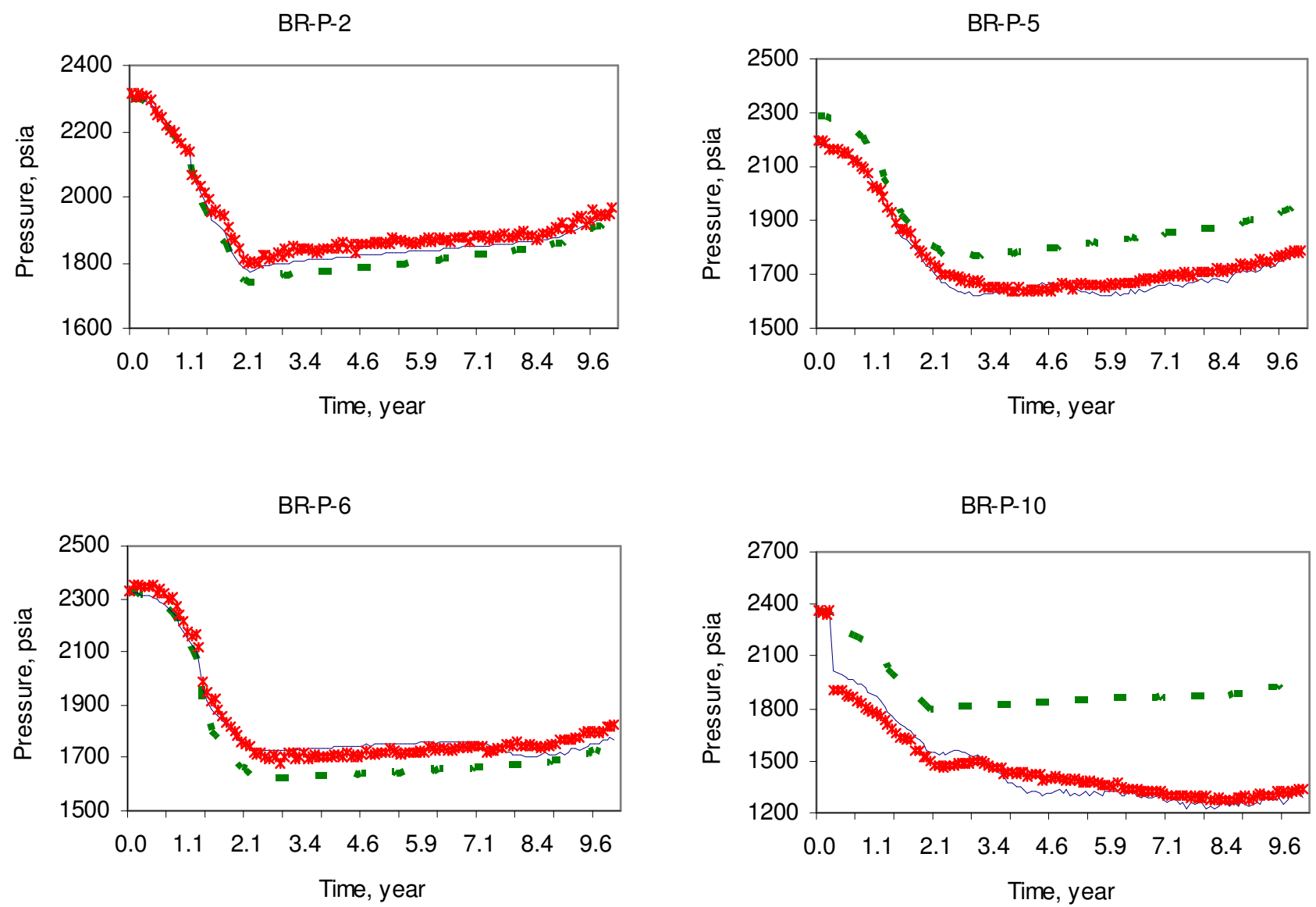

(a) Comparison for the first group of wells

Figure 4.9 Comparisons between observed, initial, and matched FBHP for multiple wells: Brugge field 

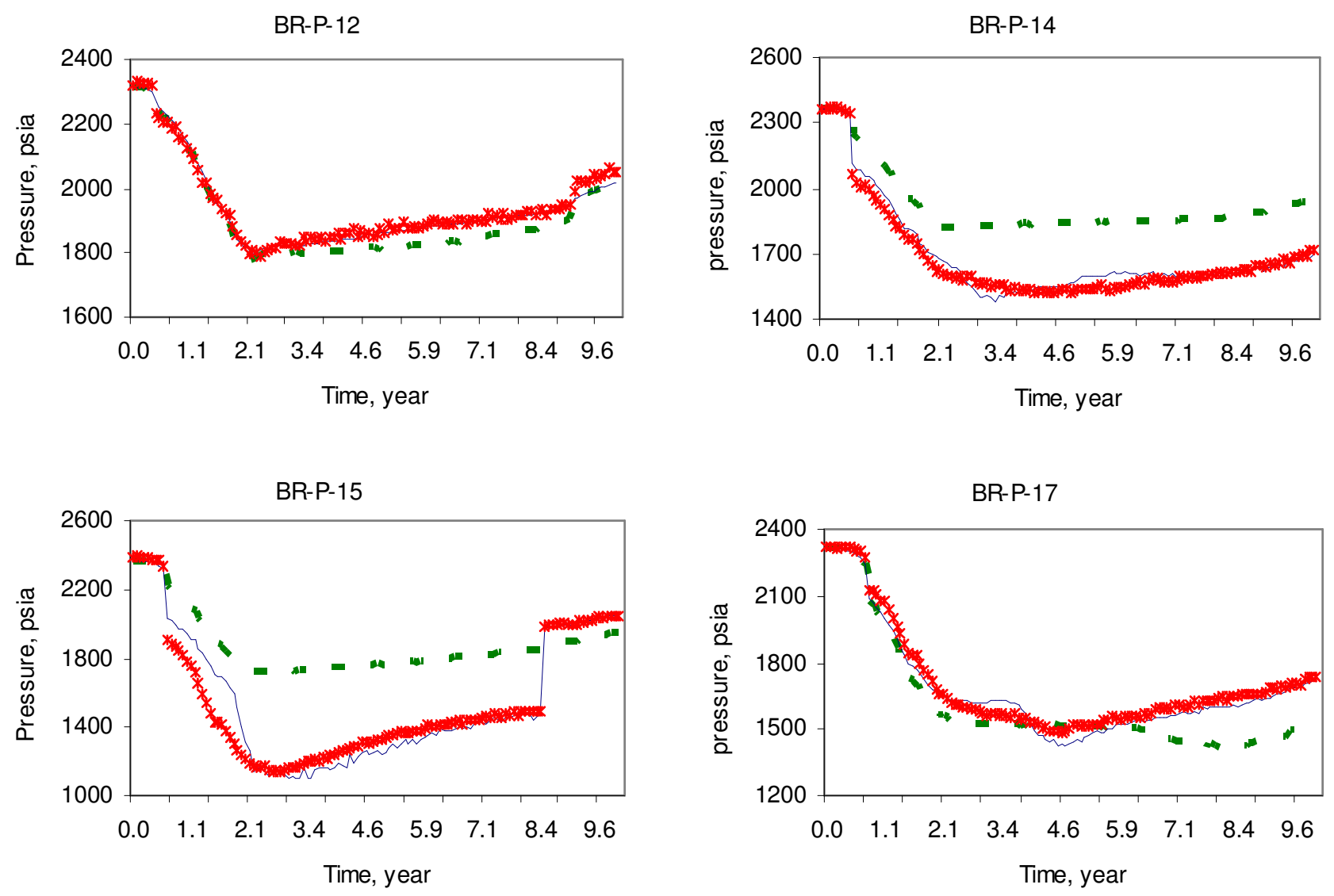

* Observed - - - Initial — Matched

(b) Comparison for the second group of wells

Figure 4.9 Continued 
Optimization Process: the optimization is performed to improve sweep efficiency over 10 history matched models by equalizing the arrival times at the producers. Most of the wells are equipped with three ICVs and the optimization is implemented by controlling the rates of the ICVs. For optimization purposes, we divided the wells into two groups based on the location of the internal fault. Group 1 includes the following producing wells: BR-P-1, 3, 4, 5, 6, 7, 8, 16, 17, 18, 19, 20 and the following injection wells: BR-I-1, 2, 3, 4, 5, and 6. Group 2 has the following producing wells: BR-P-2, 9, 10, 11, 12, 13, 14, and 15, and the following injection wells: BR-I-7, 8, 9, and 10 .

The additional constraints imposed are as follows,

- The maximum production rate per producer is $3000 \mathrm{rb} / \mathrm{Day}$.

- The maximum Injection rate per injector is $4000 \mathrm{rb} /$ Day.

- Maximum allowable flowing bottom-hole pressure is 2626 psia.

- The minimum allowable flowing bottom-hole pressure is 740 psia.

- The optimized rates are reported at each $1 / 2$ a year.

We have preformed the optimization under two scenarios. The first one imposes voidage replacement constraint and maintains the reservoir pressure. To accomplish this, we imposed the constraint that the injection for each well group is equal to the total production from the same group. The production strategy for the base case assumes injection in all ICVs in injection wells and production from all ICVs in the production wells. In addition, any production ICV with water cut exceeding $90 \%$ is shut in. Figure 4.10 shows a comparison between the base case and the optimized case in terms of average cumulative oil and water production over 20 realizations. These are the historymatched realizations that were not included in the optimization process. 


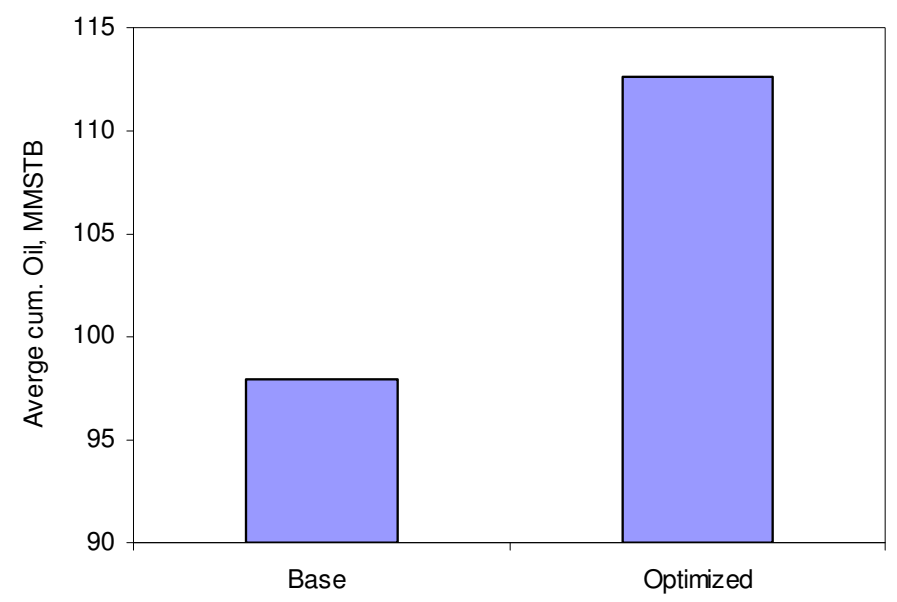

(a) Average cumulative oil production

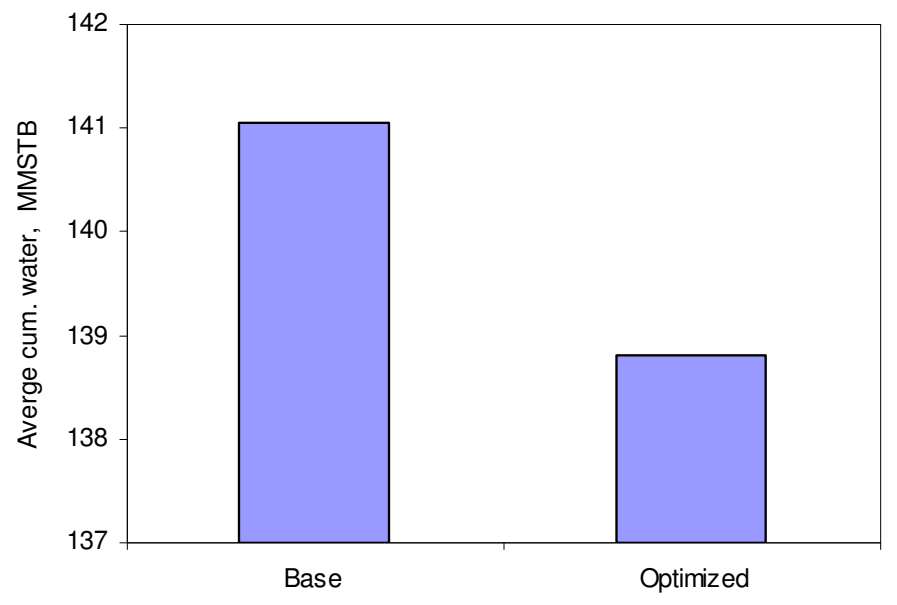

(b) Average Cumulative water production

Figure 4.10 Comparison between the base case and the optimized case in terms of average cumulative oil and water productions: Brugge field, Scenario 1

The results show an increase in the cumulative oil production and a corresponding decrease in the cumulative water production. For both the base and the optimized case, the total field injection and production was $40000 \mathrm{RB} / \mathrm{D}$ for the whole period. 
The second scenario does not impose voidage balance and attempts to produce the field at its maximum allowable production rate (60000 RB/D). The production here falls off gradually because of FBHP and water cut constraints. This scenario addresses the fact that some operators might prefer an accelerated production strategy to maximize the net present value. The total injection for this case is constrained to $40000 \mathrm{RB} / \mathrm{D}$ for the whole optimization period. This scenario has the same well constraints as the previous scenario. Figure 4.11 shows a comparison between the base and the optimized case for this scenario in terms of cumulative oil production and water production. The results indicate a noticeable increase in oil production and a substantial decrease in water production. It is important to point out that the major difference between the two scenarios is reservoir pressure maintenance strategy. Figure 4.12 shows a comparison between both scenarios in terms of reservoir pressure and total production. The total injection rate is the same for both scenarios and is equal to $40000 \mathrm{RB} / \mathrm{D}$ for the whole period as mentioned earlier. The reservoir pressure for the first scenario is maintained at its initial level because the production/injection ratio is kept close to unity and the reservoir fluids are nearly incompressible. 


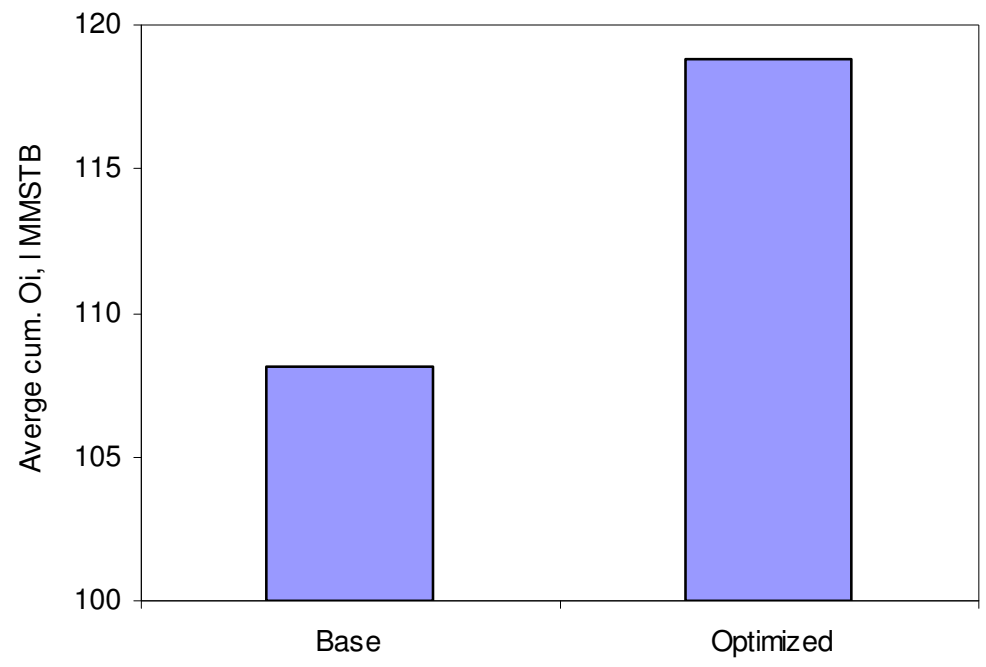

(a) Average cumulative oil production

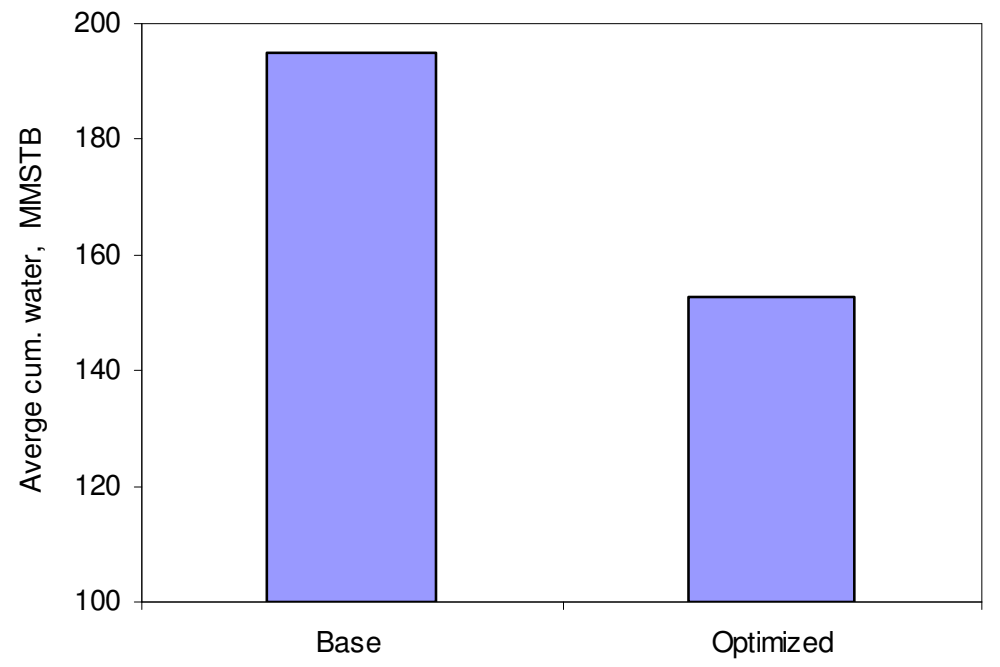

(b) Average Cumulative water production

Figure 4.11 Comparison between the base case and the optimized case in terms of average cumulative oil and water productions from 20 realizations: Brugge field, Scenario 2 


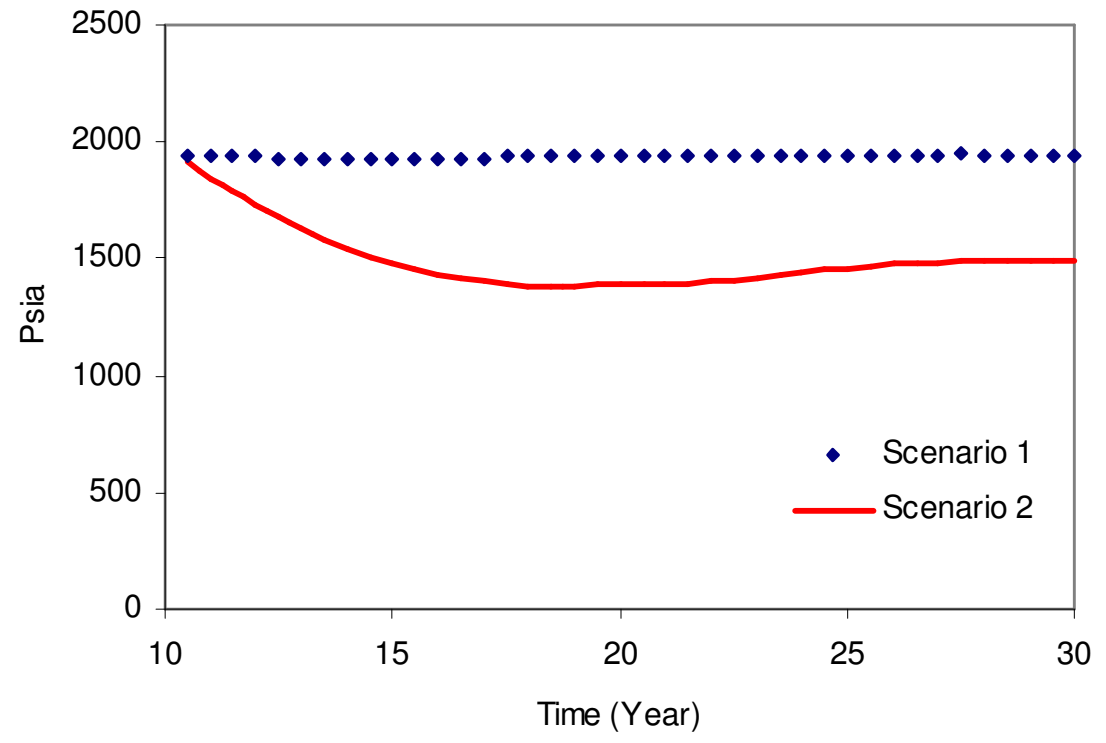

(a) Reservoir pressure

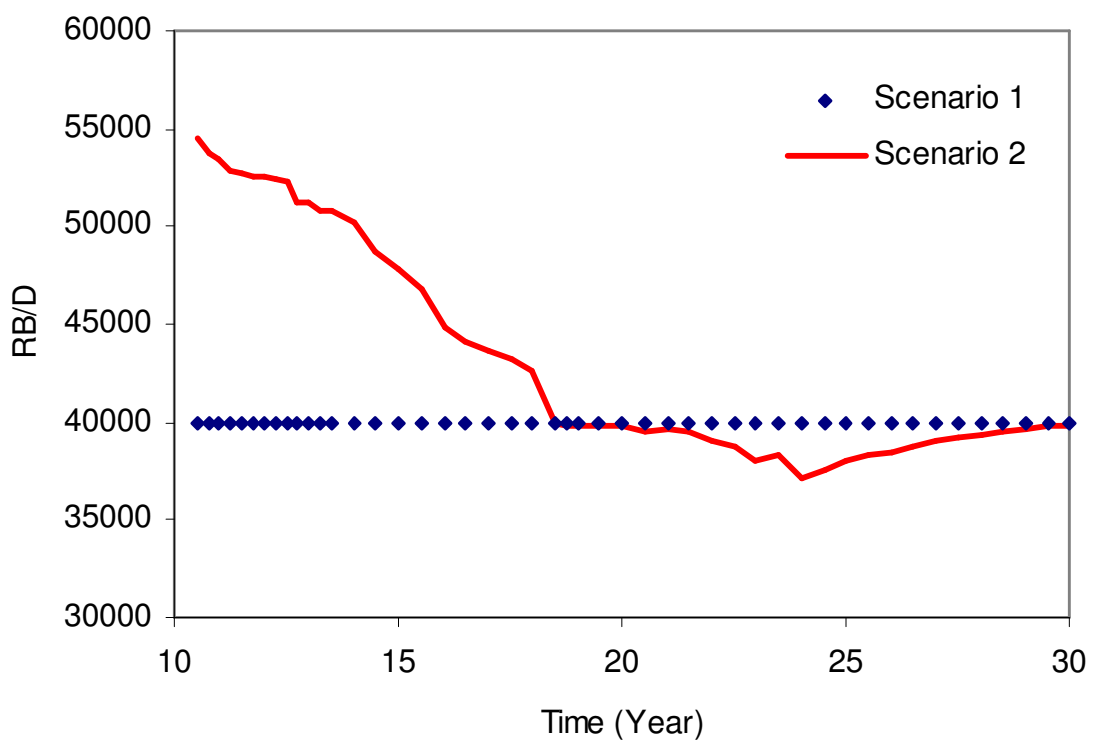

(b) Total field production rate

Figure 4.12 Comparison between scenario 1 and scenario 2 in terms of reservoir pressure and total field production rate: Brugge field 
The reservoir pressure for the second scenario falls off sharply at the beginning because the production/injection ration is greater than 1 to start with and then the pressure levels off when the production/injection ratio becomes nearly 1 . The optimized rate control profile for these two scenario are different, especially during the early production period as depicted in Figure 4.13. This figure shows the rate control strategy for producing well BR-P-1 for both scenarios. During the early time the production from ICV-1 remains almost stable in scenario 1, while it falls off quickly in scenario 2. This can be attributed to the fact that the reservoir pressure falls off quickly in the layers covered by ICV-1 for the second scenario because of the accelerated production during the early time. The rest of the profile is almost the same for all ICVs for this well.

As we discussed earlier, our optimization approach attempts to maximize the sweep efficiency. This is demonstrated in Figure 4.14 which compares the base and the optimized cases in term of oil saturation maps for layers 4 and 5 for the first scenario. The results indicate improved sweep for the optimized case over the base case as indicated by circles.

\subsubsection{Field Application in Giant Middle-Eastern Oil Field}

In this section, we will demonstrate the applicability of our approach using a giant Middle Eastern field.

Reservoir Models. The study area represents one of the most mature parts of this field with over 50 years of production history. Peripheral water injection was initiated in the late 1960s to provide full pressure maintenance. The water injection was initially implemented by gravity water injection which was replaced by sea water injection to increase flexibility in controlling the waterflood front propagation. The field development took multiple stages which were implemented in a southward trend. This area was mainly developed by vertical wells with $1 \mathrm{~km}$ spacing. All wells are equipped with surface chokes to provide flexibility in controlling wells production and injection rates. Horizontal drilling has been used extensively in the development process during the last 10 years. 


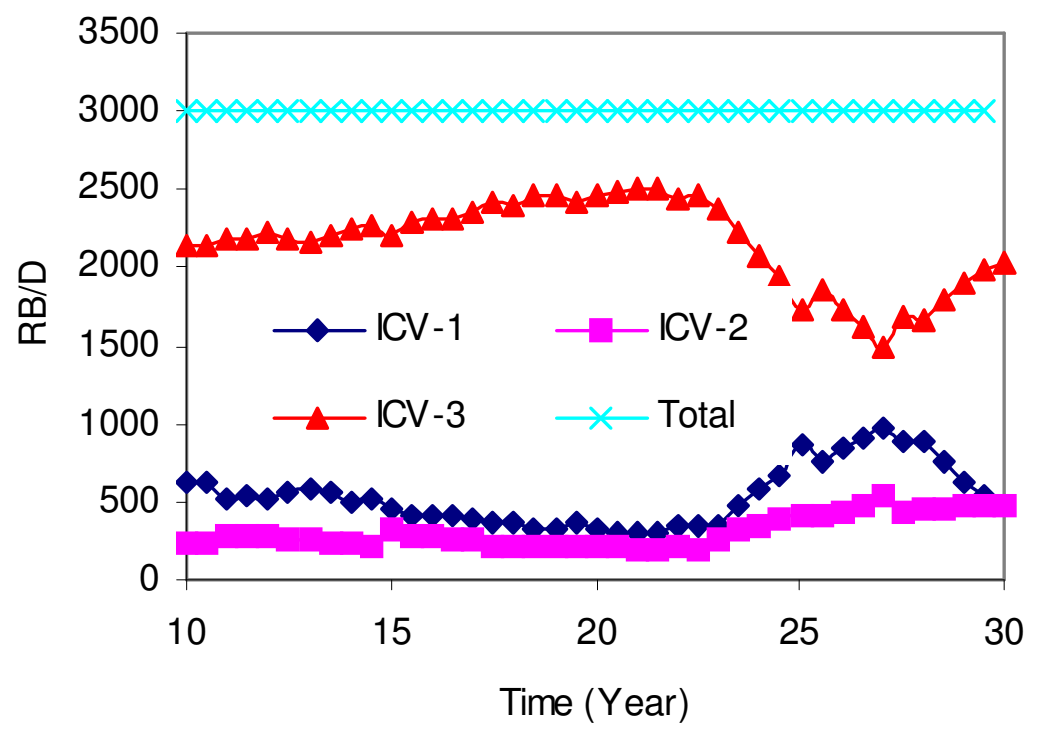

(a) Scenario 1

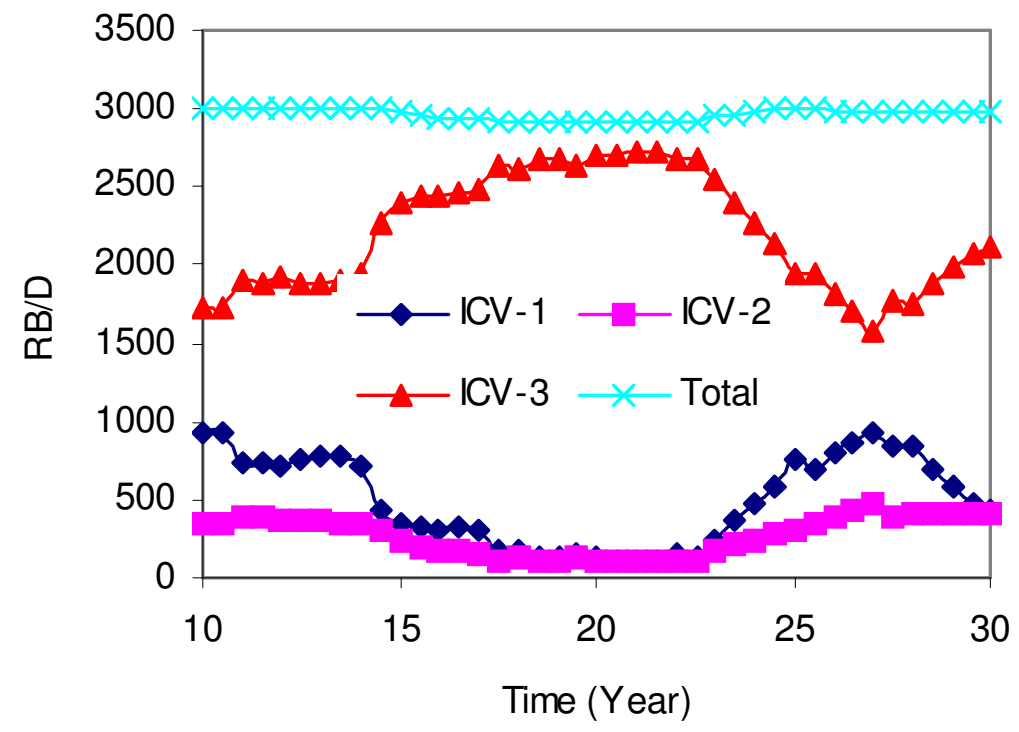

(b) Scenario 2

Figure 4.13 BR-P-1 rate performance over 20 years for scenario 1 and 2: Brugge field 


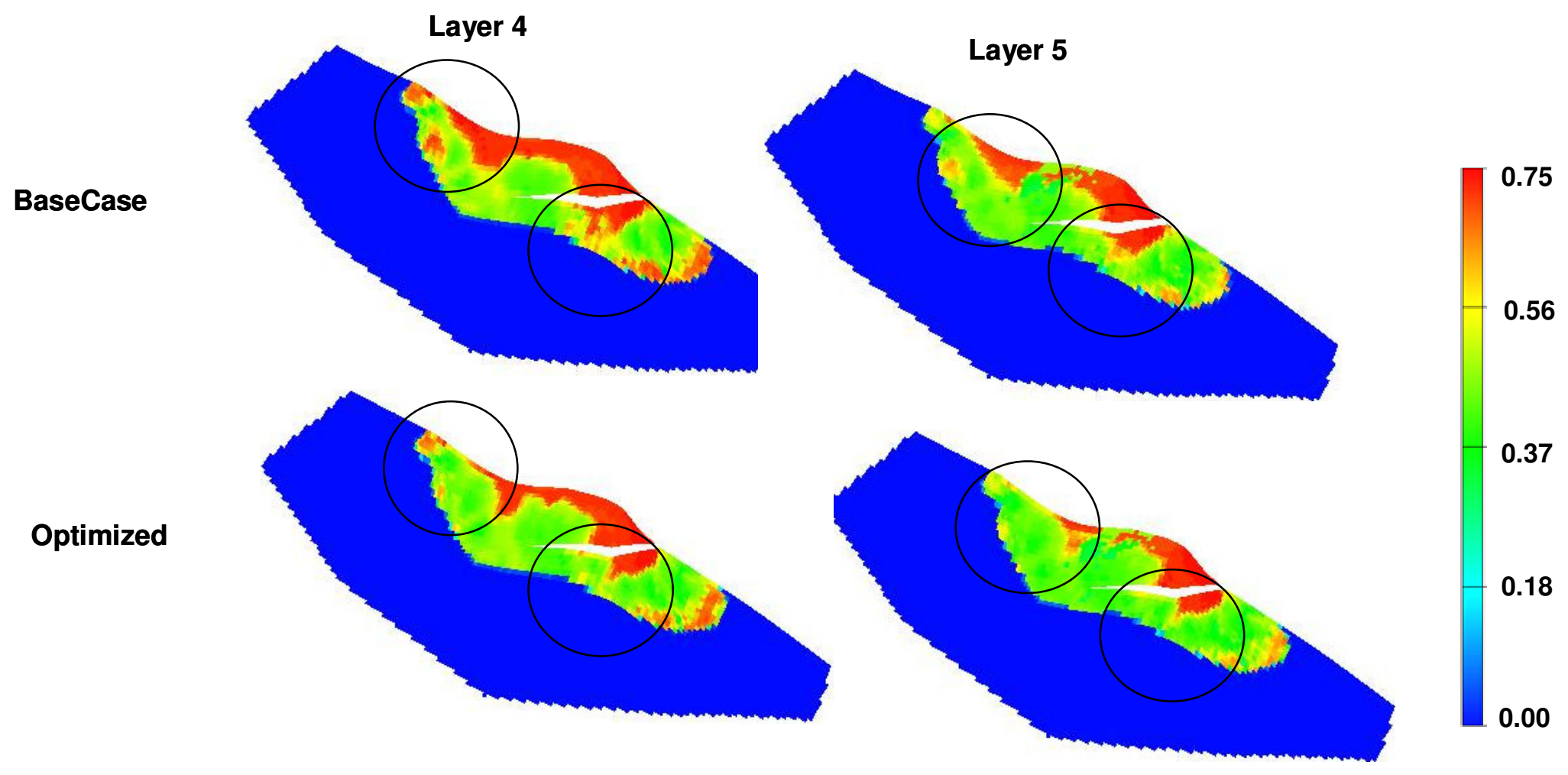

Figure 4.14 Comparisons between the base case and the optimized case in terms oil saturation for layer 4 and 5: Brugge field 
Multilateral and maximum reservoir contact wells are also being considered as a viable option to develop specific areas in the field. The main drive behind multilateral and MRC wells is to increase oil production, reduce the average water cut, reduce the unit cost of production, and utilize the limited drainage area available ( Alhuthali et al. 2005; Husain et al. 2005; Saleri et al. 2003).

The study area considered here is described as two N-S trending structural anticlines separated by a structural saddle. It is part of the Arab-D formation which consists primarily of Jurassic carbonate. The simulation model was a sector from a large field consisting of pseudo injection and production at the boundary of the sector. This sector model consisted of 90 rows, 140 columns, and 17 layers with grid cells approximately $500 \mathrm{~m}$ by $500 \mathrm{~m}$ (Ghori et al. 2007).

Optimization Process: The Optimization is performed on a sub-region of the sector model. However the sub-region includes involve a large number of wells to demonstrate the capability of our technique. Also, the optimization region includes the core area which is the farthest from the injection line. It is important to show that rates can be allocated without adversely impact the pressure performance of this area. Furthermore, there are some additional constraints are imposed throughout the optimization process such as

- Minimum and maximum allowable flowing bottom hole pressure

- Maximum and minimum well rate

- Voidage replacement for pressure maintenance

- Restrictions on high water cut wells.

To select the sub-region for optimization purposes, we employed two techniques. The first one depends on the streamline configurations. We looked at the streamlines generated in the sector model, and then chose the area within the white box as shown in Figure 4.15. 


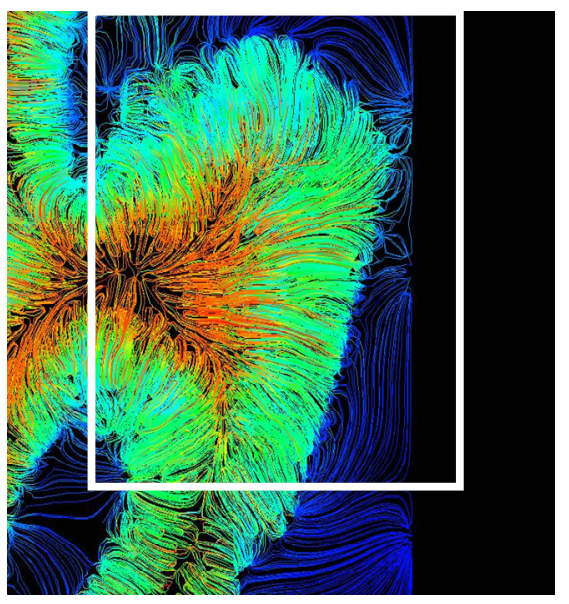

\section{Figure 4.15 Streamlines configuration, area of interest is within the white box: giant field example}

This technique gives us a general idea about where we should select our region; however, it is not adequate to specify exactly the wells to be chosen. So, we moved to a second scheme whereby we divided the injection wells in to multiple groups based on the proximity to each other as shown in Figure4.16 (a). We then computed the sensitivity of the arrival time to all producers with respect to each injector in the sector model. More details on the calculation of the arrival time sensitivity with respect to injection rates are presented in Chapter II. We then compared the magnitude of the sensitivities and assigned producers to the injection group with the highest impact on the arrival time as shown in Figure 4.16 (b). Then, we selected the injectors and producers within groups 3,4,5,6,7, and 8. Figure 4.17 shows the well locations for the selected subregion which include almost 300 wells. Most of those wells are verticals and there are a few horizontal wells. This region also includes a smart MRC well. This well consists of one lateral extending for almost $5 \mathrm{~km}$ and divided into 4 segments. Each segment is 
equipped with an ICV to control the segment production. The well is located in an area where there is an indication of stagnation as depicted by Figure 4.18.

The optimization is carried out for 20 years with an optimization time step of $1 / 2$ year. The base case has the following characteristics: (i) field will be produced at the current strategy for the next 20 years, (ii) wells will be shut in if the water cut exceed $95 \%$, (iii) no new wells are drilled during the 20 years, (iv) no change of completion is implemented during the 20 years, and (v) a ratio of 1 for voidage replacement is ensured for the area of interest. The base case will be used as benchmark to compare our optimization. It shouldn't be interpreted as if the operator will maintain the current strategy for the next 20 years. The optimization is performed under the constraint that the total injection should be the same as the base case which is dictated by the operational and facility constraints. This constraint is acknowledged during the optimization as shown in Figure 4.19 (a). There is a significant increase in the cumulative oil production for the optimized case compared to the base case as shown in Figure 4.19(b). Similarly, there is a considerable reduction in the cumulative water production as shown in Figure 4.19(c). 


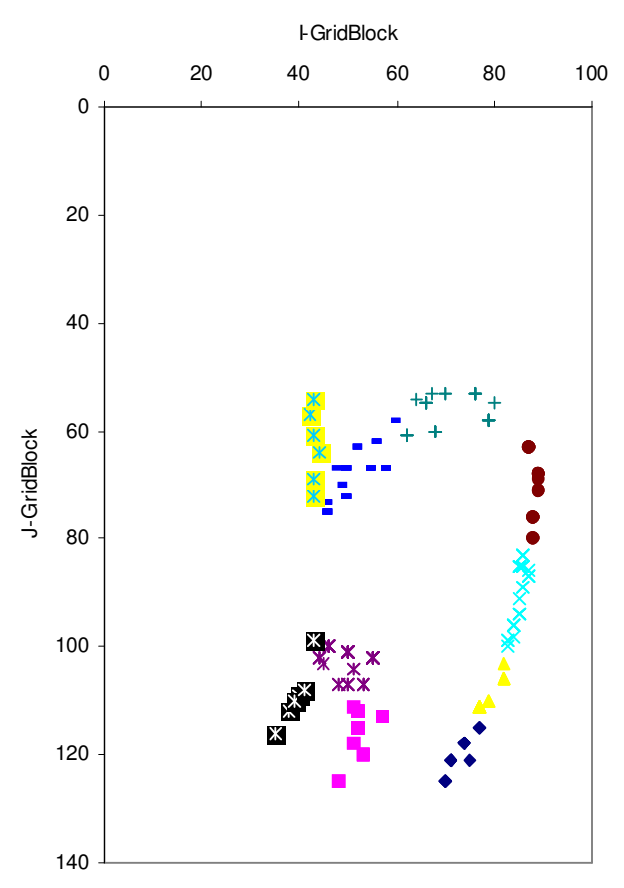

a. Injectors grouping

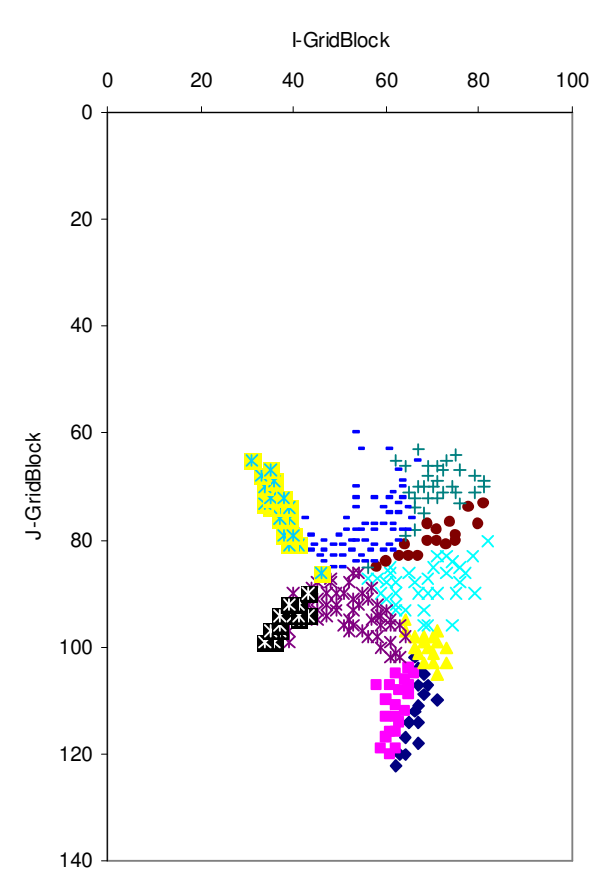

b. Producers grouping

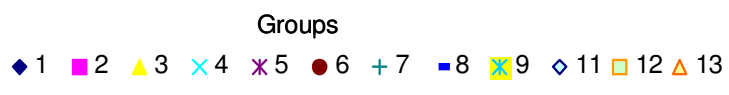

$\times 14 \mathbb{*} 15 \cdot 16+17 \boldsymbol{\Psi} 18=10$

Figure 4.16 Injectors groups and the corresponding producers groups: giant field example; some of the groups are hidden per field operator request 


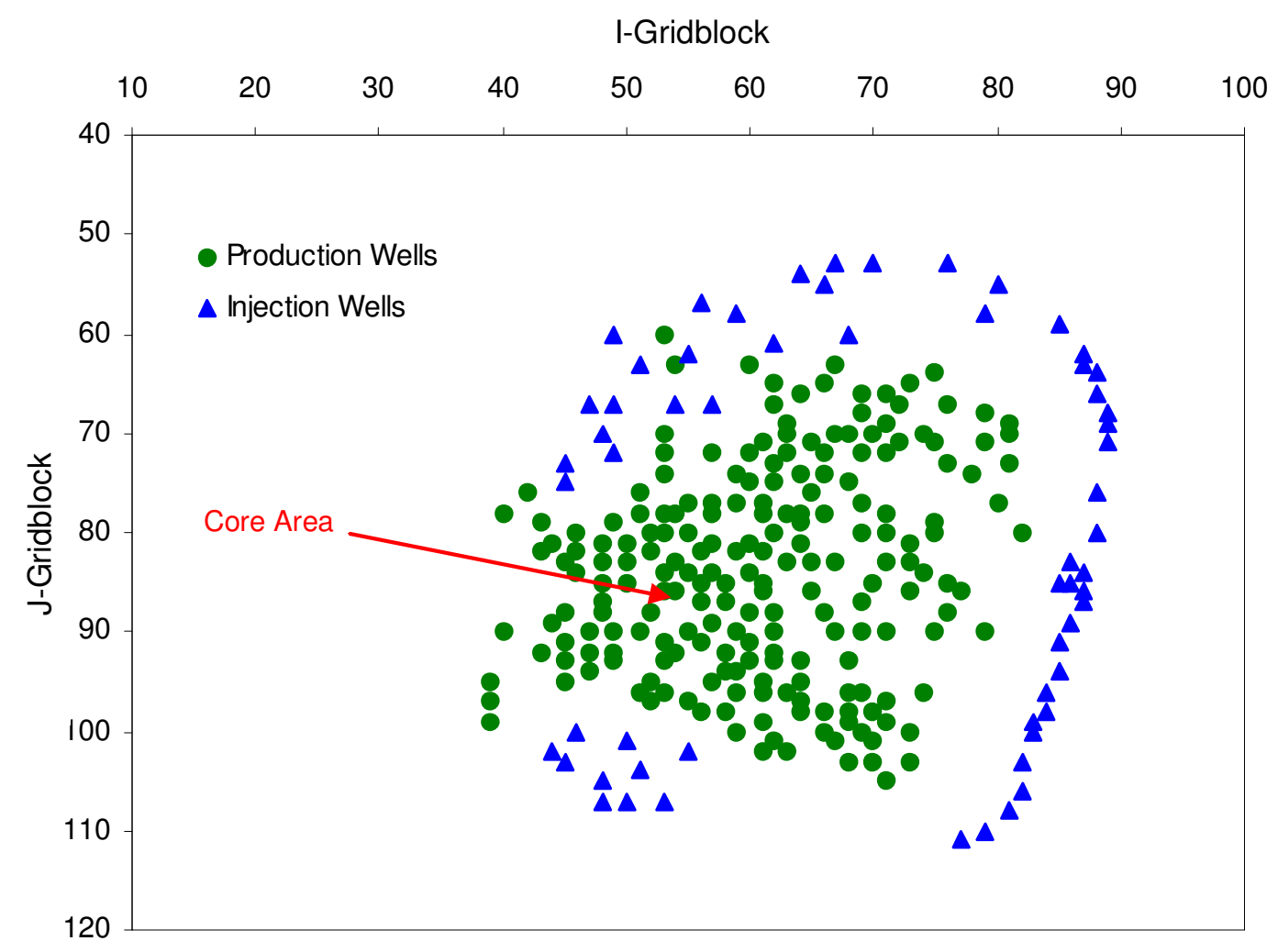

Figure 4.17 Well locations, area of interest: giant field example 


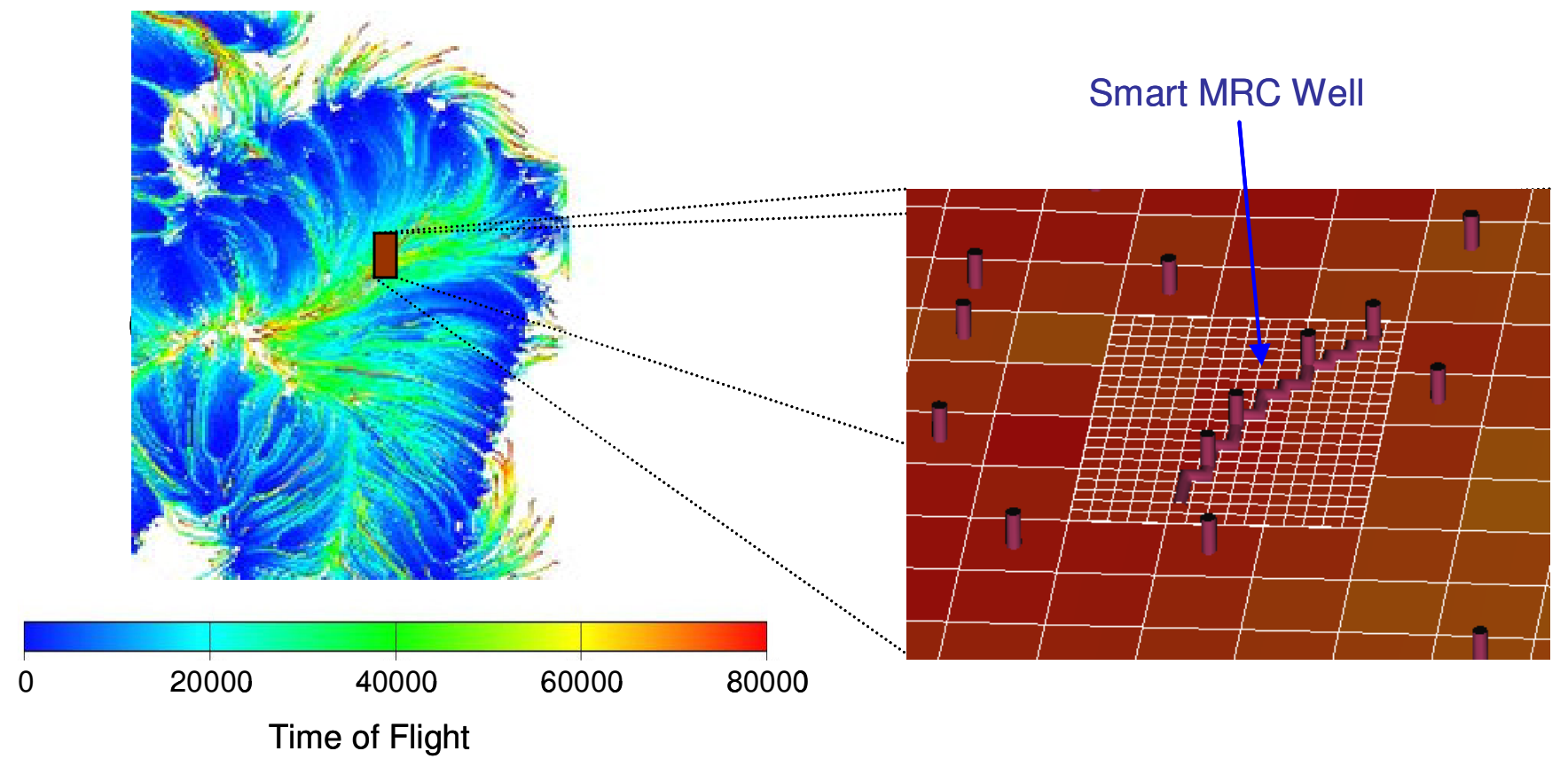

Figure 4.18 Time of flight in the area of interest, Smart MRC well location and completion: giant field example 


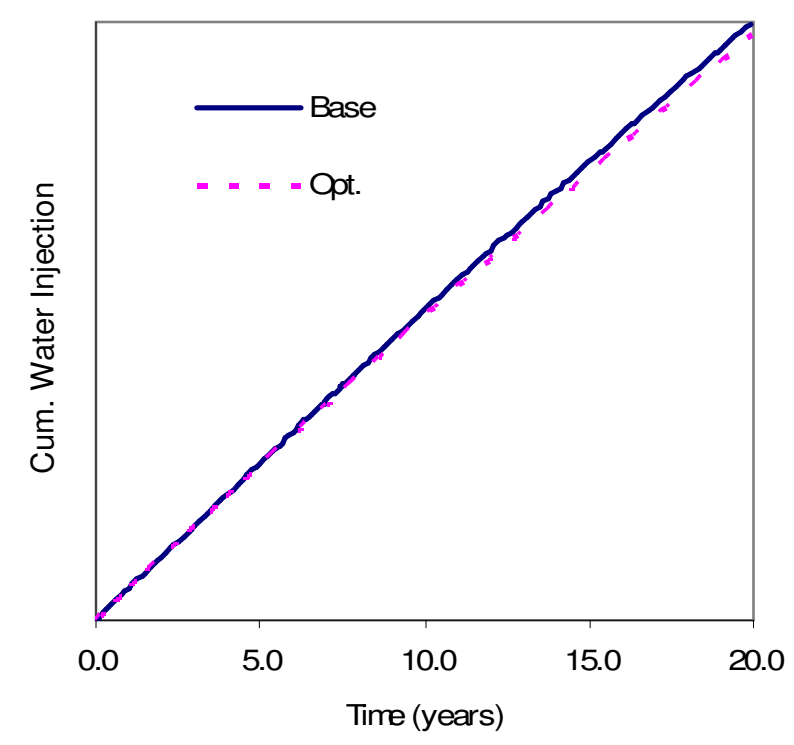

a. Cumulative water injection

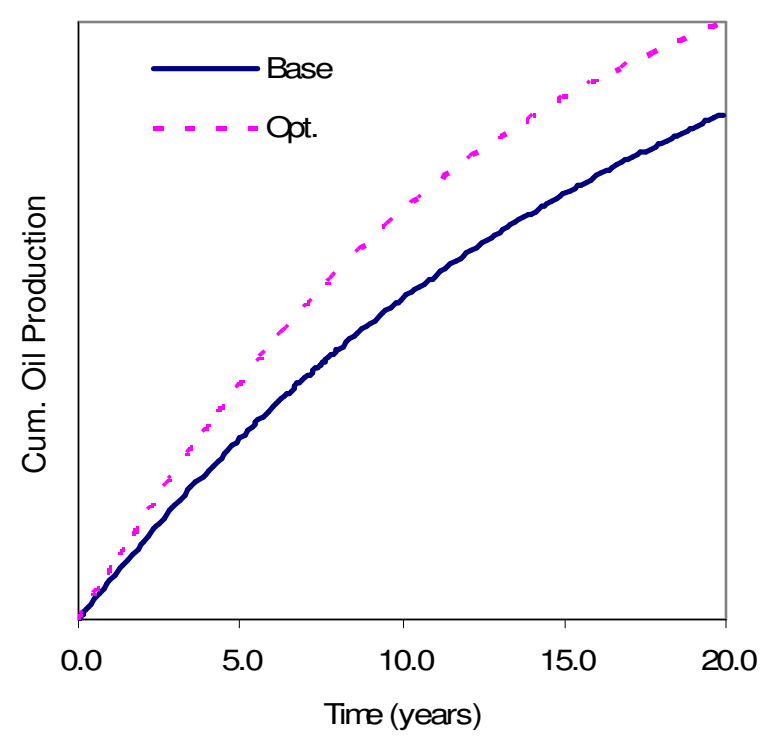

b. Cumulative oil production

Figure 4.19 Comparison between the base and the optimized case in terms of cumulative water injection, oil production, and water production; $y$-axis scale is removed per operator request 


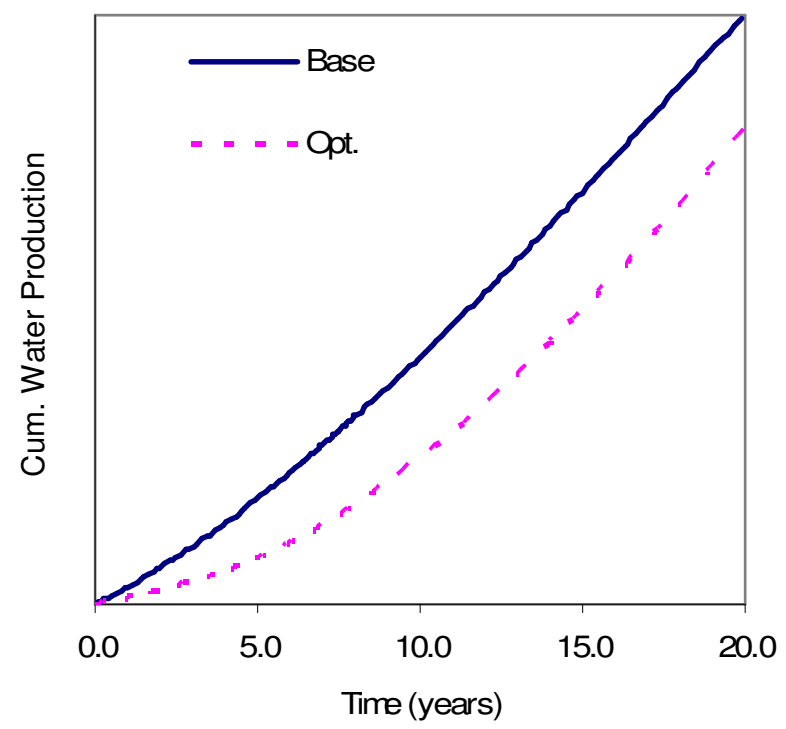

\section{c. Cumulative water production}

\section{Figure 4.19 Continued}

Figure 4.20 shows a comparison between the optimized case and the based case in terms of production and injection allocation for the first time step. This figure shows how the production and injection is reallocated after optimization. The results indicate that most of the production is re-allocated towards the core area (A-1) and the stagnation area (A-2). Figure 4.21 shows comparisons between the performance of the smart MRC well before and after the optimization. We assumed equal rates for each ICV in the base case. This figure shows an incremental oil production and a decrease in water production for the next 20 years. 


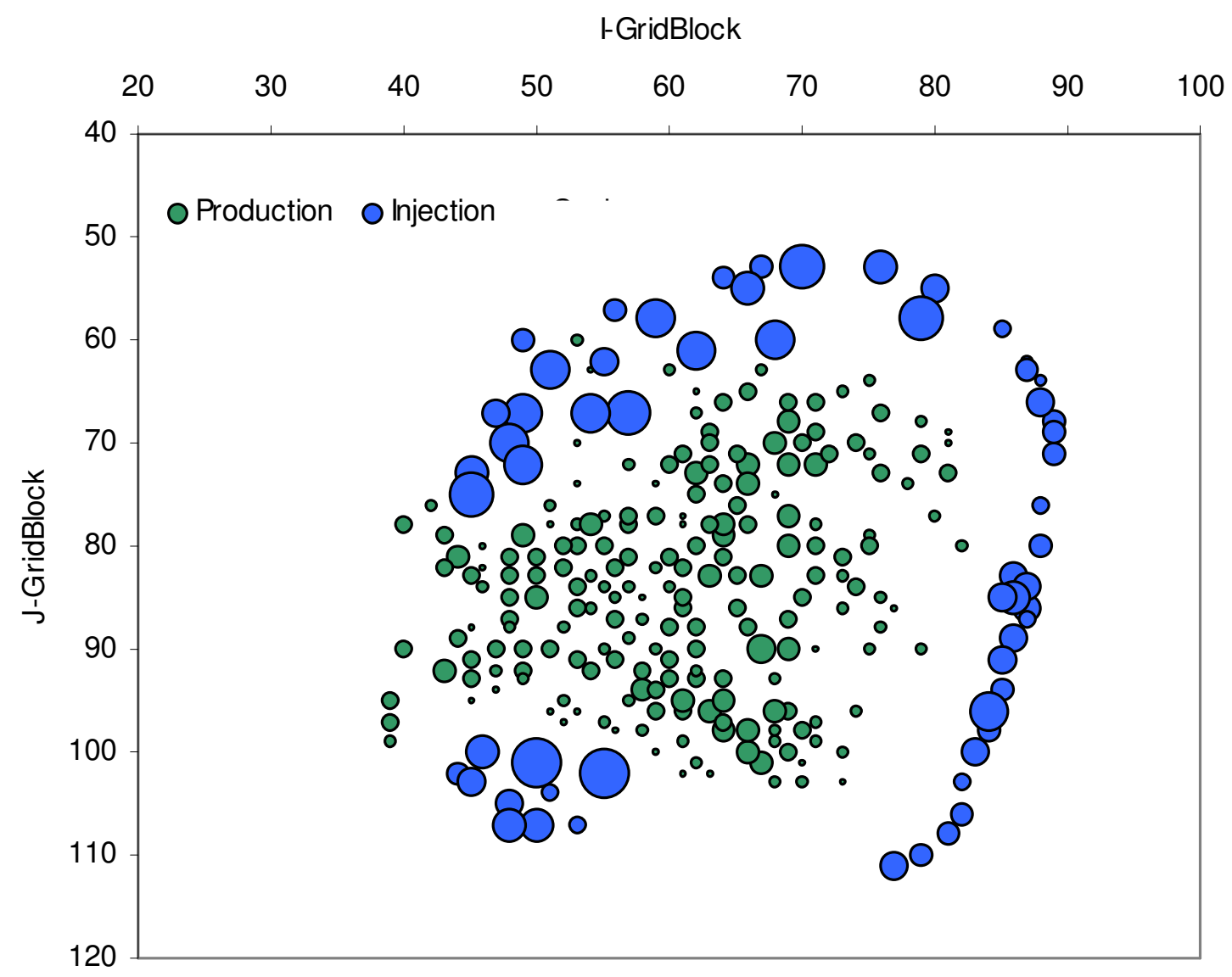

a. Base Case

Figure 4.20 Bubble maps which compare the base and the optimized cases in terms of production rates and injection rates per well at the first time step: giant field example 


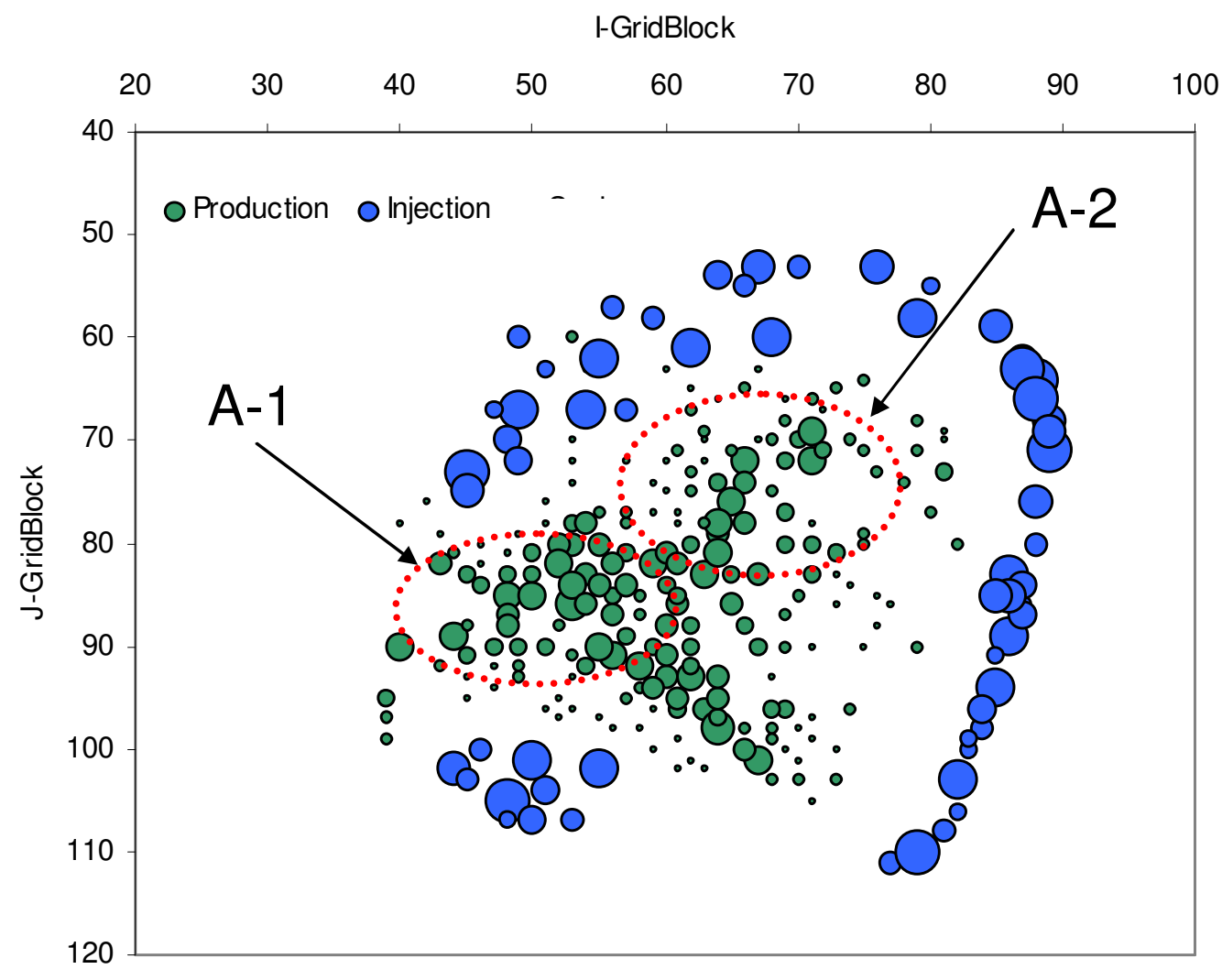

b. Optimized Case

Figure 4.20 Continued 


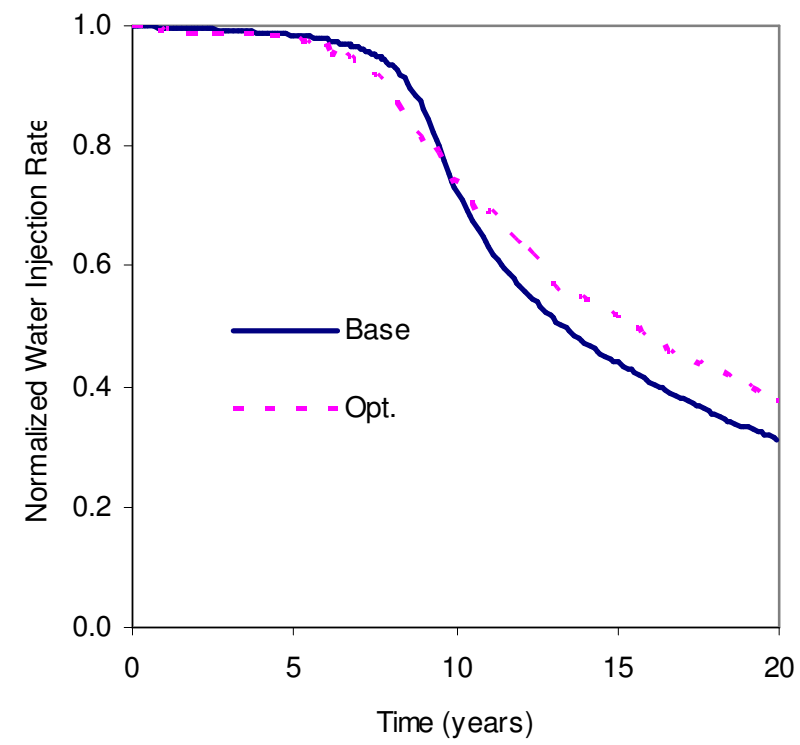

a. Normalized oil rate

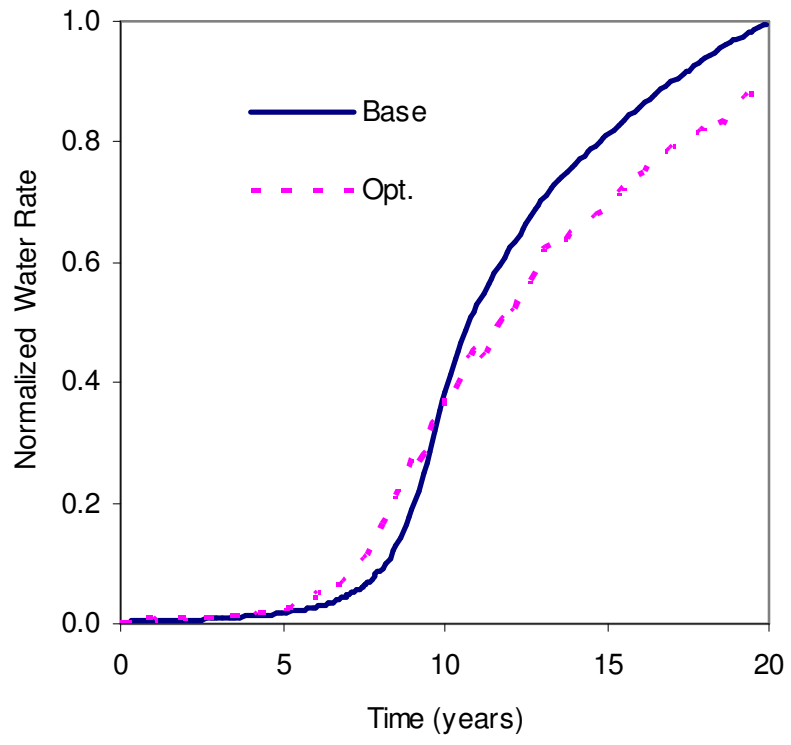

b. Normalized water rate

Figure 4.21 Comparison between the base and optimized cases for the smart MRC well in terms of oil and water rates and oil and water cumulative: giant field example 


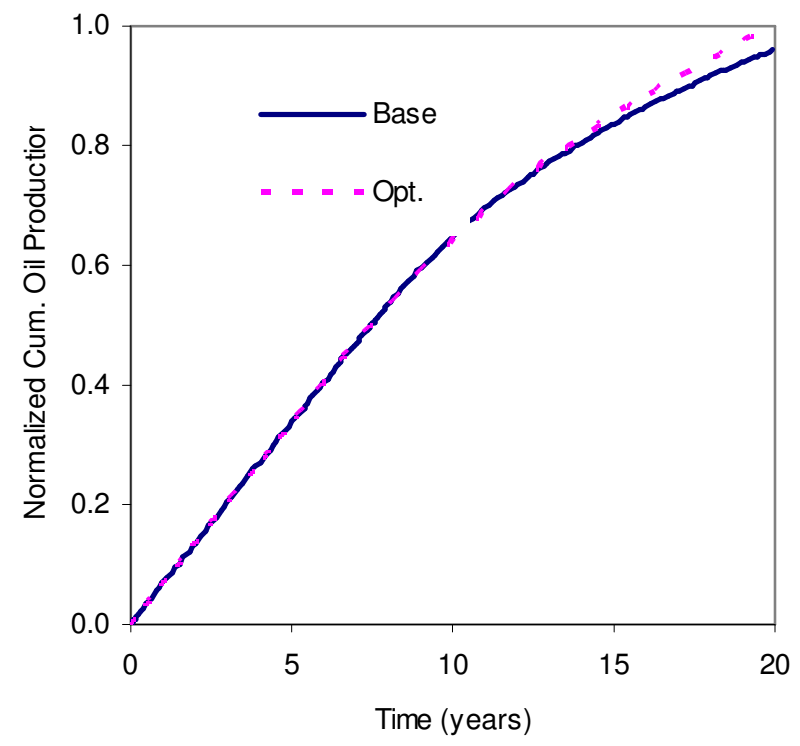

c. Normalized cumulative oil production

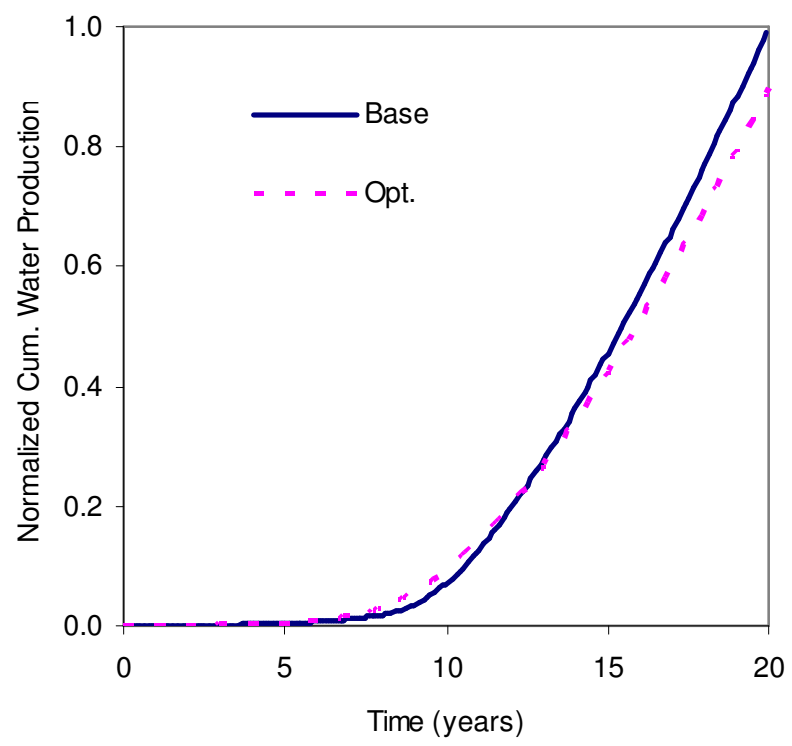

d. Normalized cumulative water production

Figure 4.21 Continued 
The pressure maintenance in the core area A-1 was not compromised because the optimization was performed under flowing bottom hole pressure constraints. Figure 4.22 shows the pressure performance of two wells within the core area, indicating that the flowing BHP constraints are maintained.

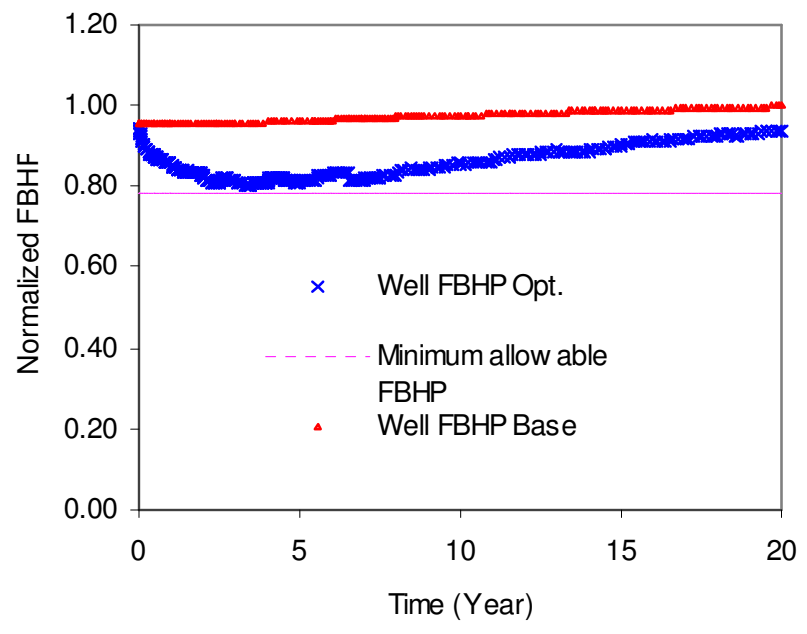

a. Well 1

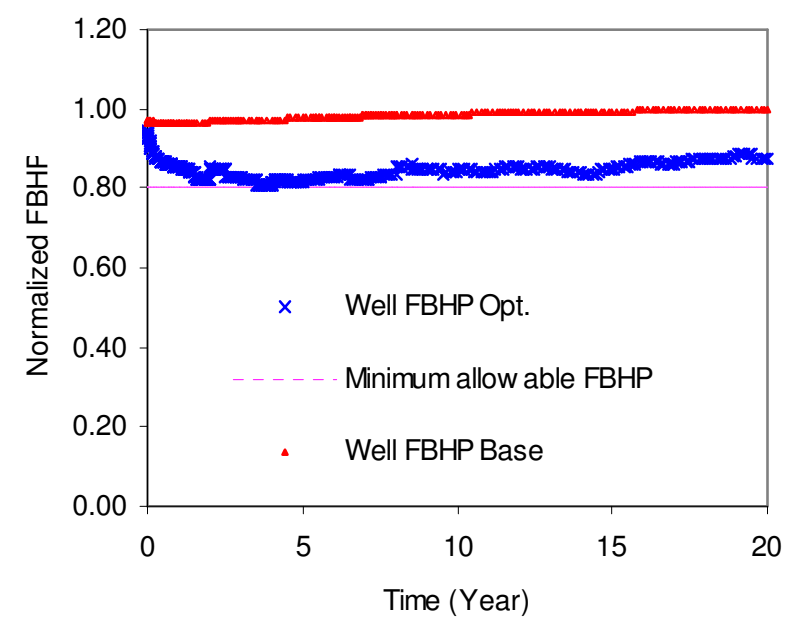

b. Well 2

Figure 4.22 Comparisons between the base and the optimized cases in terms of the FBHP for two wells located in the core area: giant field example 


\subsection{Chapter Summary}

In this chapter, we demonstrated via field applications an efficient approach to waterflood optimization using smart wells and optimal rate control. Our approach relies on equalizing the streamline time of flight at the producing wells to maximize waterflood sweep efficiency. We also demonstrated the ability of our approach to handle some of the non-linear constraints that arise in managing a water flood project such as minimum and maximum allowable FBHP and restriction on high water cut wells. The optimization approach calls for allocating injection/production rates to maximize the sweep efficiency during waterflood and can account for geologic uncertainty using multiple realizations. We show that the approach is computationally efficient and yields robust rates that can be implemented in field operations using two 3D field-scale examples.

The first example is a synthetic case based on a North Sea Brent-type field called 'Brugge' field. The optimization was part of a closed loop approach which consisted of two steps: (i) production data integration for the first 10 years of production history, and (ii) production optimization over the next 20 years. This process was performed on multiple realizations to account for geologic uncertainty. The rates derived from the optimization process were tested using multiple history matched realizations that were not included during the optimization. Results showed that the optimized rates significantly improved sweep efficiency and oil recovery compared to the base case.

In the second example, the optimization was used to improve the sweep efficiency in a giant Middle-Eastern field. The optimization involved nearly 300 vertical and horizontal wells and one smart MRC well. Results showed significant improvements in cumulative oil production and a corresponding decrease in water production using the optimized rates compared to the base production strategy. 


\section{CHAPTER V}

\section{CONCLUSIONS AND RECOMMENDATIONS}

\subsection{Conclusions}

Field scale rate optimization problems often involve highly complex reservoir models, production and facilities related constraints and a large number of unknowns. All these make optimal reservoir management via rate and flood front control difficult without efficient optimization tools. Some aspects of the optimization problem have been studied before using mainly optimal control theory. However, the applications todate have been limited to rather small problems because of the computation time and the complexities associated with the formulation and solution of adjoint equations. Fieldscale rate optimization for maximizing waterflood sweep efficiency under realistic field conditions has still remained largely unexplored.

The primary objective of our optimization algorithm is to enhance the sweep efficiency in a waterflood project by equalizing the arrival time at multiple producers and correspondingly increase the cumulative oil production. There are four major characteristics that distinguish our approach from previously published works. First, we use streamlines to efficiently and analytically compute the sensitivity of the arrival times with respect to well rates. Second, we account for geologic uncertainty via two optimization frameworks: (i) a stochastic framework that relies on a combination of the expect value and variance of a performance measure from multiple realizations for risk assessments and (ii) a min-max problem formulation that minimizes the worst case scenario. Third, analytical forms for gradients and Hessian of the objective functions are derived which make our optimization computationally efficient for large field cases. Finally, the optimization is performed under operational and facility constraints using a sequential quadratic programming approach. We proposed a measurement-based optimization that allows us to assign specific weights to each realization involved in the optimization process based on observed data. Our approached is general and can be 
employed using both finite-deference and streamline models. In addition, the approach can be implemented on multiple geologic realizations to account for geologic uncertainty. We assume that these realizations are readily available and we limit the optimization to well rates only. In general, the optimization will be performed at multiple time steps to account for mobility effects, changing field conditions and nonlinear constraints. This is illustrated in Figure 5.1.

During each optimization time-step, the following steps are implemented:

- Flow simulation and streamline tracing. The first step is to perform a flow simulation for a specific time interval for each geologic realization. Either a streamline or a finite difference simulator can be used here. Streamlines will be readily available if a streamline simulator is used. However, if a finite-difference simulator is used, then we need to trace the streamlines using the fluid fluxes derived from the flow simulation (Datta-Gupta and King, 2007).

- Compute the Travel Time Residuals and Analytical gradient and Hessian. The second step is to compute the residuals which quantify the difference between the desired arrival time and the computed arrival time at each producing well. In addition, this step involves the analytical computation of gradients and the Hessian using a streamline-based technique (Alhuthali et al., 2008).

- Minimization and Optimal Rate Allocations. Once the gradient and the Hessian are available, we can use standard optimization algorithms to minimize the objective function. In our approach, we use the Sequential Quadratic Programming (SQP) technique (Nocedal and Wright 2006) to generate the required changes in rates to minimize the objective function subject to appropriate field constraints. The above-mentioned steps are repeated until a predefined stopping criterion on the objective function or the rates is met. We then move on to the next time interval for optimization. 

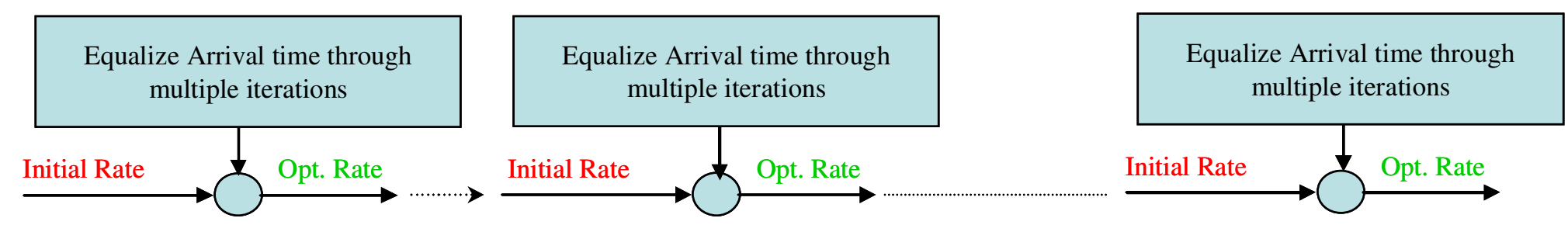

Opt. Tstep 1

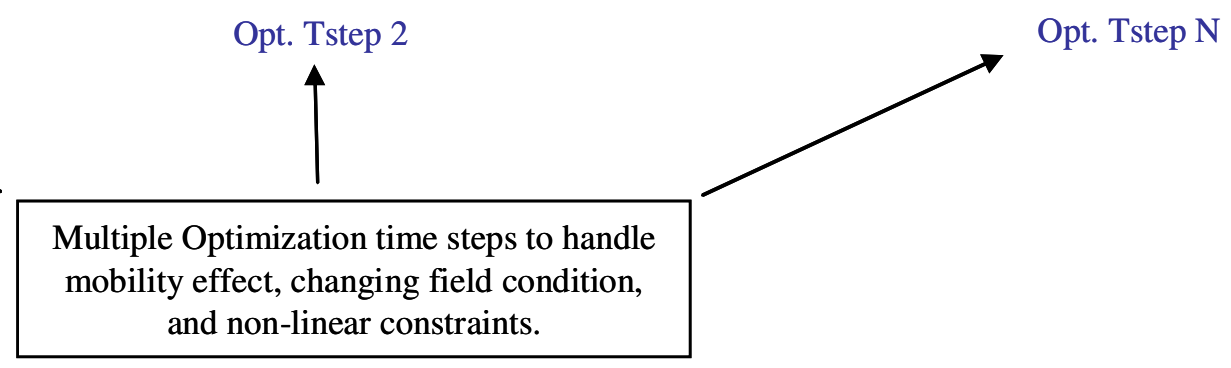

Figure 5.1 Flow chart to show the optimization process at multiple time steps 
Some specific conclusions from this study can be summarized as follows:

1. Streamline time of flight provides an effective way to optimize waterflood through equalizing the arrival time at the producers. The streamline approach also facilitates analytical computation of the arrival time sensitivities with respect to production and injection rates.

2. Analytical computation of sensitivities requires only one flow simulation regardless of the number of injectors and producers and leads to an efficient optimization algorithm. The assumptions used to derive the analytical sensitivities were verified by comparing the results with the numerical perturbation method.

3. Geologic uncertainty was addressed in terms of two forms of objective functions: (i) a stochastic form which includes the expected value and the standard deviation combined with a risk coefficient and (ii) a min-max form which minimizes the worst case scenario.

4. Analytical formulations of the objective function gradients and Hessian were derived. These formulations require only one flow simulation per realization regardless of the number of injectors and producers and leads to an efficient optimization algorithm.

5. The proposed measurement-based optimization provides a simple mechanism to incorporate production history during optimization.

6. We have validated our approach using $2 \mathrm{D}$ homogenous and heterogeneous cases and also by application to smart wells with inflow control valves. In all these cases, the optimization has led to a significant delay in water breakthrough with corresponding increase in sweep efficiency and oil recovery.

7. The power and practical feasibility of our method has been demonstrated by a field example from the Goldsmith San Andres Unit (GSAU) in west Texas. We optimized the production rates for 31 wells while accounting for changing field condition such as shut-in and infill drilling. Also, we show that the approach is computationally efficient and yields robust rates that can be implemented in field 
operations using two 3D field-scale examples (Alhuthali et al. 2009). The first example is a synthetic case based on a North Sea Brent-type field called 'Brugge' field. The optimization was part of a closed loop approach which consisted of two steps: (i) production data integration for the first 10 years of production history, and (ii) production optimization over the next 20 years. This process was performed on multiple realizations to account for geologic uncertainty. The rates derived from the optimization process were tested using multiple history matched realizations that were not included during the optimization. Results showed that the optimized rates significantly improved sweep efficiency and oil recovery compared to the base case. In the second example, the optimization was used to improve the sweep efficiency in a giant Middle-Eastern field. The optimization involved nearly 300 vertical and horizontal wells and one smart MRC well. Results showed significant improvements in cumulative oil production and a corresponding decrease in water production using the optimized rates compared to the base production strategy.

8. In all studied cases, our approach improved sweep efficiency and increased oil production with a corresponding decrease in the water-cut response.

\subsection{Recommendations}

Several recommendations that could improve the performance of the proposed optimization algorithm or extend the applications of the presented concepts are listed below:

1. The current optimization algorithm relies mainly on equalizing the arrival time at all producer. Water cut is not considered in the formulation of the objective function; however, we allowed for restricting high water cut wells as bound constraints in the optimization process. This approach was discussed in Chapter IV. In general the optimization algorithm doesn't guarantee restricting high water cut wells especially if the reason for high water production is not related to high the permeability streaks. For example, the water production can be high because 
the well is located in a mature area. Equalizing the arrival time in a mature field might yield an extensive water production. Here, we suggest a methodology by which we can include the water cut in the formulation of the objective function and prevent allocating high production rates to high water cut wells. To achieve this objective, the arrival time is modified to have the following form:

$t_{i, m}^{\prime}(\mathbf{q})=\left(1-w_{c, i, m}\right) t_{i, m}(\mathbf{q})$

In the above expression, the arrival time, $t_{i, m}$, at well $i$ which belongs to group $m$ is multiplied by the well's oil cut. If the water cut is zero, the modified arrival time is equal the original arrival time. When the water cut is greater than zero, the original arrival time will be rescaled based on the level of water cut. If the water cut is high, the original arrival time will be reduced significantly and this will prevent increasing the well's production rate.

If we assume that the water cut in Eq. 5.1 is not a function of the well's production rate, a single element in the sensitivity matrix can be written as,

$$
S_{i j}^{\prime}=\frac{\partial t_{i, m}^{\prime}(\mathbf{q})}{\partial q_{j}}=\left(1-w_{c, i, m}\right) \frac{\partial t_{i, m}(\mathbf{q})}{\partial q_{j}}=\left(1-w_{c, i, m}\right) S_{i j}
$$

The variables $S_{i j}^{\prime}$ and $S_{i j}$ represent the sensitivity element of the modified and the original arrival time respectively. The rest of the derivation of the objective function and its minimization can be carried on in a similar way which was presented in the previous chapters.

2. A natural extension of the work presented in this study is to implement it on a $\mathrm{CO}_{2}$ Flooding. The presence of high permeability streaks can adversely impact the efficiency of $\mathrm{CO}_{2}$ Flooding leading to an early gas breakthrough and uneven flooding process. Here, we suggest extending our method to equalize the arrival time of $\mathrm{CO}_{2}$. We believe that the extension is straight forward and require the following steps: 
- Tracing streamlines and arrival time computation. The first step is to generate streamlines and compute the time of flight. Tracing streamlines in $\mathrm{CO}_{2}$ has been discussed by.

- Residuals and Sensitivity Computation. In this step we compute the residuals that quantify the difference between the desired arrival time and the computed arrival time at each of the producing well. We also calculate the sensitivity of the arrival time at the producer to wells rates analytically using simple integrals along streamlines.

- Minimization and Optimal Rate Allocations. Sequential quadratic programming (SQP) procedures is used to minimize the arrival time residuals. This step generates the required changes in rates to equalize gas front arrival time at the producing wells subject to appropriate field constraints.

3. The current optimization algorithm doesn't account for accelerated production strategy to maximize the net present value. We suggest adding a norm constraint to the objective function to take care of the acceleration effect. The objective function can be written for a single realization as follows,

$\min f(\mathbf{q})=\mathbf{e}^{T} \mathbf{e}+\mathbf{t}^{T} \mathbf{t}$

q

Subject to

$$
\begin{aligned}
& \mathbf{h}(\mathbf{q})=0 \\
& \mathbf{g}(\mathbf{q}) \leq 0
\end{aligned}
$$

Where $h: \mathfrak{R}^{n} \rightarrow \mathfrak{R}^{z}$ and $g: \mathfrak{R}^{n} \rightarrow \mathfrak{R}^{y}$

The vector $\mathbf{t}$ in Eq. 5.3 represent the total arrival time and minimizing the second term in the above equation will account for acceleration effect. The gradient of this objective has the following form,

$\nabla_{q}\left[\mathbf{e}^{T} \mathbf{e}\right]=2\left[\mathbf{J}^{T} \mathbf{e}+\mathbf{S}^{T} \mathbf{t}\right]$. 
Recall that $\mathbf{J}$ is the Jacobian of the misfit, and $\mathbf{S}$ is the sensitivity of the arrival time. The Hessian can be written as,

$$
\nabla_{q}^{2}\left[\mathbf{e}^{T} \mathbf{e}+\mathbf{t}^{T} \mathbf{t}\right]=2\left[\mathbf{J}^{T} \mathbf{J}+\mathbf{S}^{T} \mathbf{S}\right]
$$

It is important to point out that those forms can be generalized easily to multiple realizations using the same procedures illustrated in Appendices B and C. 


\section{NOMENCLATURE}

A

A

$B_{o}$

$B_{w}$

b

C

$d$

d

$\delta \mathbf{d}$

$f(\mathbf{q})$

$f_{w}$

e

$e_{i, m}$

$\mathbf{g}(\mathbf{q})$

$\mathbf{h}(\mathbf{q})$

I

$i$ and $j$

$k$

$\mathbf{J}$

area along the streamline, $\mathrm{sp} \mathrm{ft}\left[\mathrm{m}^{2}\right]$

matrix contains linear operators

oil FVF, STB/bbl [stock-tank $\mathrm{m}^{3} / \mathrm{m}^{3}$ ]

water FVF, STB/bbl [stock-tank $\mathrm{m}^{3} / \mathrm{m}^{3}$ ]

vector contains constant elements

matrix contains linear operators

observed field measurements

vector contains constant elements

vector of generalized travel time shift of water cut and zero frequency pressure misfit.

scalar objective function, sq day

fractional flow, dimensionless

arrival time residual vector, day [s]

arrival time residual at well $i$ (producer) which belong to group $m$, day [s]

inequality constrains

equality constrains

identity matrix

well index

permeability, darcies

Jacobian matrix 


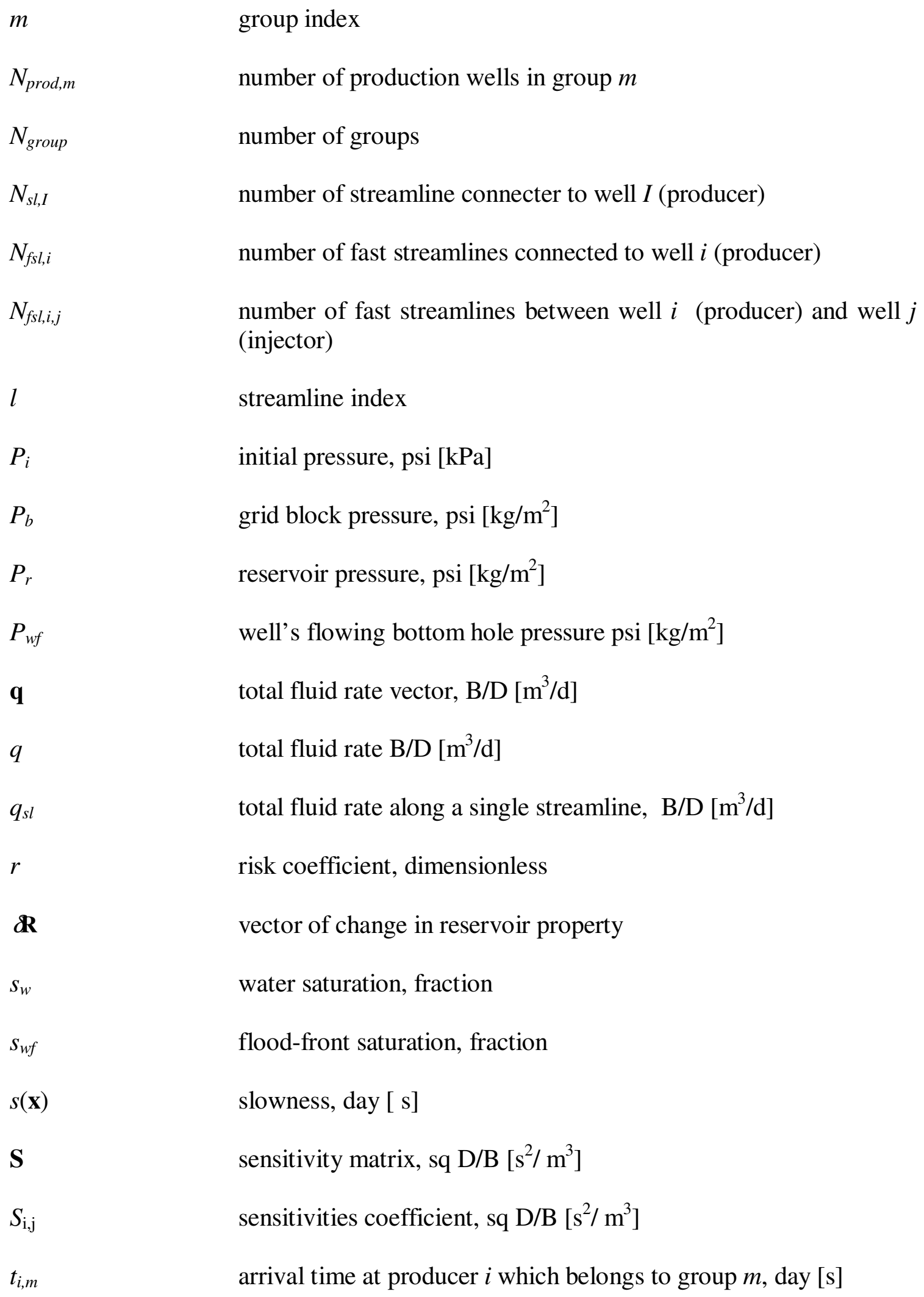




\begin{tabular}{ll}
$t_{d, m}$ & desired arrival time for group $m$, day [s] \\
$\mathrm{v}$ & velocity, $\mathrm{ft} / \mathrm{D}[\mathrm{m} / \mathrm{d}]$ \\
$w$ & weight, dimensionless \\
$w_{c, i, m}$ & water cut at producer $i$ which belongs to group $m$, day [s] \\
$\mathbf{x}$ & spatial coordinate vector, $\mathrm{ft}[\mathrm{m}]$ \\
$x$ & distance along the streamline, $\mathrm{ft}[\mathrm{m}]$ \\
$X$ & random variable represent model parameters \\
$y$ & number of inequality constraints \\
$z$ & number of equality constraints \\
$\lambda_{L}$ & Lagrange multipliers for equality constraints \\
$\lambda_{K}$ & Karush-Kuhn-Tucker multipliers for inequality constraints \\
$\beta$ & weighting factor \\
$\mu_{o}$ & oil viscosity, cp [Pa $\bullet \mathrm{s}]$ \\
$\mu_{w}$ & water viscosity, cp [Pa $\bullet \mathrm{s}]$ \\
$\rho$ & isk tolerance \\
$\rho_{o}$ & water viscosity, lbm $/ \mathrm{cu} \mathrm{ft}\left[\mathrm{kg} / \mathrm{m}^{3}\right]$ \\
$\rho_{w}$ & water viscosity, $\mathrm{lbm} / \mathrm{cu} \mathrm{ft}\left[\mathrm{kg} / \mathrm{m}^{3}\right]$ \\
\hline & porosity, fraction \\
\hline &
\end{tabular}




\section{REFERENCES}

Al-Harbi, M., Cheng, H., He, Z., and Datta-Gupta, A. 2005. Streamline-Based Production Data Integration in Naturally Fractured Reservoirs. SPEJ 10 (4): 426439. SPE: 89914-PA. DOI: 10.2118/89914-PA.

Alhuthali, A.H., Al-Awam, H.H., Soremi, Y., and Al-Towailib, A.I. 2005. Water Management in North 'Ain Dar, Saudi Arabia. Paper SPE 93439 presented at the Middle East Oil \& Gas Show and Conference, Bahrain, 12-15 March. DOI: 10.2118/93439-MS.

Alhuthali, A.H., Oyerinde, D., and Datta-Gupta, A. 2007. Optimal Waterflood Management Using Rate Control. SPEREE 10 (5): 539-551. SPE: 102478-PA. DOI: 10.2118/102478-PA.

Alhuthali, A.H., Datta-Gupta, A., Yuen, B, and Fontanilla, J.P. 2008. Optimal Rate Control Under Geologic Uncertainty. Paper SPE 113628 presented at the SPE/DOE Symposium on Improved Oil Recovery. DOI: 10.2118/113628-MS.

Alhuthali, A.H., Datta-Gupta, A., Yuen, B, and Fontanilla, J.P. 2009. Field Applications of Waterflood Optimization via Optimal Rate Control With Smart Wells. Paper SPE 118948 presented at the SPE Reservoir Simulation Symposium, The Woodlands, Texas, 2-4 February. DOI: 10.2118/118948-MS.

Arenas A. and Dolle N. 2003. Smart Waterflooding Tight Fractured Reservoirs Using Inflow Control Valves. Paper SPE 84193 presented at the SPE Annual Technical Conference and Exhibition, Denver, Colorado, 5-8 October. DOI: 10.2118/84193MS.

Asheim, H. 1988. Maximization of Water Sweep Efficiency by Controlling Production and Injection Rates. Paper SPE 18365 presented at the SPE European Petroleum Conference, London, 16-18 October. DOI:102118/18365-MS.

Bickel, J. Eric, Richard L. Gibson, Duane A. McVay, Stephen Pickering, and John Waggoner. 2006. Quantifying the Reliability and Value of 3D Land Seismic. SPEREE 11 (5): 832-841. SPE: 102340-PA. DOI: 10.2118/102340-PA. 
Brayton, R.K., Director, S.W., Hachtel, G.D., and Vidigal, L., 1979. A New Algorithm for Statistical Circuit Design Based on Quasi-Newton Methods and Function Splitting. IEEE Trans. Circuits and Systems CAS-26 (9): 784-794.

Brouwer, D.R., Jansen, J.D., Van der Starre, S., van Kruijsdijk, C.P.J.W. and Berentsen, C.W.J. 2001. Recovery Increase Through Waterflooding With Smart Well Technology. Paper SPE 68979 presented at the SPE European Formation Damage Conference, The Hague, 21-22 May. DOI: 10.2118/68979-MS.

Brouwer, D. R., Jansen, J.D. 2004. Dynamic Optimization of Waterflooding With Smart Wells Using Optimal Control Theory. SPEJ 9 (4):391-402. SPE: 78278-PA. DOI: 10.2118/78278-PA.

Chen, Y., Oliver, D.S., and Zhang, D. 2008. Efficient Ensemble-based Closed-Loop Production Optimization. Paper SPE 112873 presented at the SPE/DOE Improved Oil Recovery Symposium, Tulsa, Oklahoma, 19-23 April. DOI: 10.2118/112873MS.

Cheng. H., Kharghoria. A., He, Z., and Datta-Gupta, A. 2005. Fast History Matching of Finite-Difference Models Using Streamline-Derived Sensitivities. SPEREE 8 (5): 426-436. SPE: 89447-PA. DOI: 10.2118/89447-PA.

Cheng, H. Datta-Gupta, A., and He, Z. 2005. A Comparison of Travel-Time and Amplitude Matching for Field-Scale Production-Data Integration: Sensitivity, Nonlinearity, and Practical Implications. SPEJ 10 (1): 75-90. SPE: 84570-PA. DOI: 10.2118/84570-PA.

Craig, Forrest F. 1971. The Reservoir Engineering Aspects of Waterflooding, Richardson, Texas. Society of Petroleum engineers of AIME.

Cosentino, L. Coury, Y. Daniel, J. M., Manceau, E., Ravenne, C., Van Lingen, P. Cole, J., and Sengul, M. 2002. Integrated Study of a Fractured Middle East Reservoir With Stratiform Super-K Intervals-Part 2: Upscaling and Dual-Media Simulation. SPEREE 5 (1): 24-32. SPE: 76642-PA. DOI: 10.2118/76642-PA. 
Datta-Gupta, A. and King, M.J. 1995. A Semianalytic Approach to Tracer Flow Modeling in Heterogeneous Permeable Media. Adv. In Water Resources 18 (1): 924.

Datta-Gupta, A. and King, M. J. 2007. Streamline Simulation: Theory and Practice, Richardson, Texas. Society of Petroleum Engineers.

ECLIPSE File Formats Reference Manual 2005. Schlumberger Information Solutions.

Emerick, A.A., Portella, R.C.M., and Petrobras, S. A. 2007. Production Optimization With Intelligent Wells. Paper SPE 107261 presented at Latin American and Caribbean Petroleum Engineering Conference, Buenos Aires, Argentina, 15-18 April. DOI: 10.2118/107261-MS.

Ghori, S. G., Jilani, S. Z., Al-Huthali, A. H., Krinis, D., and Kumar, A. 2007. Improving Injector Efficiencies Using Streamline Simulation: A Case Study in a giant Middle East Field. Paper SPE 105393 presented at the Middle East Oil \& Gas Show and Conference, Kingdom of Bahrain, 11-14 March. DOI: 10.2118/105393-MS.

Glandt C. A. 2003. Reservoir Aspects of Smart Wells. Paper SPE 81107 presented at the SPE Latin American and Caribbean Petroleum Engineering Conference held in Port-of-Spain, Trinidad, West Indies, 27-30 April. DOI: 10.2118/81107-MS.

Grinestaff, G. H. and Caffrey, D. J. 2000. Water Management: A Case Study of the Northwest Fault Bock Area of Prudhoe Bay, Alaska, Using Streamline Simulation and Traditional Waterflood Analysis. Paper SPE 63152 presented at the SPE Annual Technical Conference and Exhibition, Dallas, Texas, 1-4 October. DOI: 10.2118/81107-MS.

Grinestaff, G.H. 1999. WaterFlood Pattern Allocations: Quantifying the Injector to Producer Relationship with Streamline Simulation. Paper SPE 54616 presented at the 1999 SPE Western Regional Meeting, Anchorage, Alaska, 26-28 May. DOI: 10.2118/54616-MS.

Gu, Y. and Oliver, D. S. 2007. An Iterative Ensemble Kalman Filter for Multiphase Fluid Flow Data Assimilation. SPEJ, 12 (4): 438--446. SPE: 108438-PA. DOI: 10.2118/108438-PA. 
Guyaguler, B., and Horne, R. N. 2001. Uncertainty assessment of Well Placement Optimization. Paper SPE 71625 presented at the SPE Annual Technical Conference and Exhibition, New Orleans, Louisiana, 30 September-3 October. DOI: 10.2118/71625-MS.

He, Z., Yoon, S., and Datta-Gupta, A. 2002. Streamline-based Production Data Integration With Gravity ad Changing Field Conditions. SPEJ 7 (4): 423-436. SPE: 81208-PA. DOI: 10.2118/81208-PA.

Howard, R.A. 1971. Proximal Decision Analysis. Management Science 17 (9):507-541. DOI: $10.1287 / \mathrm{mnsc}$.17.9.507.

Hussain, A., Kumar A., Garni, S.A., and Shammari, M.A. 2005. Optimizing MaximumReservoir-Contact Wells: Application to Saudi Arabian Reservoirs. Paper IPTC 10395 presented at the International Petroleum Technology Conference, Doha, Qatar, 21-23 November. DOI: 10.2523/10395-MS.

King, M.J. and Datta-Gupta, A. 1998. Streamline Simulation: A Current Perspective. In Situ 22 (1): 91-141.

McLaughlin, D. and Townley, L.R. 1996. A Reassessment of the Ground-water Inverse Problem. Water Resources Research 32 (5): 1131-1161.

Lake L W., Schmidt R. L., and Venuto P. B. 1992. A Niche for Enhanced Oil Recovery in the 1990s. Oilfield Review: Schlumberger Publication : 55-61, January.

Lorentzen, R. J., Berg M. A., Naevdal G., and Vefring, E. H. 2006. A New Approach for Dynamic Optimization of Water Flooding Problems. Paper SPE 99690 presented at the SPE Intelligent Energy Conference and Exhibition, Amsterdam, Netherlands, 11-13 April. DOI 10.2118/99690-MS.

Naevadal G., Brouwer D. R., and Jansen J. D. 2006. Waterflooding Using Closed-loop Control. Computational Geosciences 10: 37-60. DOI: 10.1007/s10596-005-9010-6.

Nocedal, J. and Wright, S. J. 2006: Numerical Optimization, 2nd ed. New York: Springer Science + Business Media. 
Osako, I., Datta-Gupta, A. and King, M.J. 2004. Timestep Selection During Streamline Simulation Through Transverse Flux Correction. SPEJ 9 (4): 459-464. SPE: 79688PA. DOI: 10.2118/79688-PA.

Paige, C.C. and Saunders, M.A. 1982.LSQR: An Algorithm for Sparse Linear Equations and Sparse Least Squares. ACM Transactions on Mathematical Software 8 (1): 4371.

Peters, L., Arts, R.J., Brouwer, G.K., and Gell, C. 2009. Results of the Brugge Benchmark Study for Flooding Optimization and History Matching. Paper SPE 119094 will be presented at the SPE Reservoir Simulation Symposium held in The Woodlands, Texas, USA, 2-4 February. DOI: 10.2118/119094-MS.

Pollock, d.W. 1988. Semianalytical Computation of Path Lines for Finite-difference Models. Ground Water 26 (6): 743-750.

Saleri, N. G., Salamy, S.P., and Al-Otaibi, S.S. 2003. The Expanding Role of the Drill Bit in Shaping the Subsurface. JPT 55 (12): 53-56. SPE: 84923-MS. DOI: 10.2118/84923-MS.

Sarma, P., Durlofsky, L. J., and Aziz, K. 2005. Efficient Closed-loop Production Optimization under Uncertainty. Paper SPE 94241 presented at the Europec/EAGE Annual Conference, Madrid, Spain, 13-16 June. DOI: 10.2118/94241-MS.

Simpson, G. S. Lamb, F. E., Finch, J. H., and Dinnie, N. C. 2000. The Application of Probabilistic and Qualitative Methods to Asset Management Decision Making. Paper SPE 59455 presented at the SPE Asia Pacific Conference on Integrated Modeling for Asset Management, Yokohama, Japan, 25-26 April. DOI: 10.2118/59455-MS.

Sudaryanto, B. and Yortsos. Y.C. 2001. Optimization of Displacements in Porous Media Using Rate Control. Paper SPE 71509 presented at the SPE Annual Technical Conference and Exhibition, New Orleans, Louisiana, 30 September-3 October. DOI: 10.2118/71509-MS.

Tavakkolian, M. Jalali, F., and Amadi, M. A. 2004. Production Optimization Using Genetic Algorithm Approach. Paper SPE 88901 presented at the Annual SPE 
International Technical Conference Exhibition, Abuja, Nigeria, 2-4 August. DOI: 10.2118/88901-MS.

Thiele, M. R. and Batycky, R. P. 2006. Using Streamline-Derived Injection Efficiencies for Improved Waterlood Management. SPEREE 9 (2): 187-196. SPE: 84080-PA. DOI: $10.2118 / 84080-P A$.

Yeten, B., Durlofsky, L.J., Aziz, K. 2003. Optimization of Nonconventional Well Type, Location, and Trajectory. SPEJ 8 (3): 200-210. SPE: 86880-PA. DOI: 10.2118/86880-PA.

Van Essen, G. M., Zandvliet, M. J., Van Den Hof P. M. J. , Bosgra, O. H., and Jansen, J. D. 2006. Robust Waterflooding Optimization of Multiple Geological Scenarios. Paper SPE 102913 presented at the Annual Technical Conference and Exhibition, San Antonio, Texas, 24-27 September. DOI: 10.2118/102913-MS.

Vasco, D.W. and Karasaki, K. 2006. Interpretation and Inversion of Low-frequency Head Observations. Water Resour. Res 42, W05408. DOI:10.1029/2005WR004445. Wang, C., Li, G. and Reynolds, A. C. 2007. Production Optimization in Closed-Loop Reservoir Management. Paper SPE 109805 presented at the SPE Annual Technical Conference and Exhibition, Anaheim, California, 11-14 Novermber. DOI: 10.2118/109805-MS. 


\section{APPENDIX A \\ DERIVATION OF THE STOCHASTIC FORM OF THE OBJECTIVE FUNCTION}

The problem of rate optimization under geologic uncertainty can be studied within the decision analysis framework. The problem involves the decision on the optimal rates given that we have multiple geologic realizations. These realizations will yield multiple events or outcomes $y_{\mathrm{i}}$ defined as,

$$
y_{i}=\mathbf{e}_{i}^{T} \mathbf{e}_{i}
$$

As Eq. A.1 indicates, the outcomes are simply the square of $l_{2}$ norms of the residuals for realization $i$. Through decision analysis framework and utility theory (Howard 1971), we can derive an equation which combines all the outcomes in a single form.

$$
f(\mathbf{q})=\mathrm{E}\left[\mathbf{e}^{T} \mathbf{e}\right]-\frac{1}{2 \rho} \operatorname{Var}\left(\mathbf{e}^{T} \mathbf{e}\right) .
$$

Equation A.2 will be minimized over all possible outcomes to decide on the optimal rates. The variable, $\rho$, is a risk tolerance which describes the decision makers' attitude towards risk. The risk tolerance has the same unit as the expected value. A positive $\rho$ indicates a risk-prone attitude, while a negative $\rho$ indicates a risk-averse attitude. The case of $\rho=\infty$ indicates that the decision maker is risk neutral. The sign interpretation of $\rho$ is different from the previously published work because our objective is to minimize Eq. A.2 not to maximize it. In general, the assessment of the risk tolerance is involved and depends on the decision maker's preferences (Guyaguler and Horne 2001). In our work, we re-write Eq. A.2 as follows,

$$
f(\mathbf{q})=\mathrm{E}\left[\mathbf{e}^{T} \mathbf{e}\right]+r \sigma\left[\mathbf{e}^{T} \mathbf{e}\right]
$$


Where we define a risk coefficient $r$ as follows

$$
r=-\frac{\sigma\left[\mathbf{e}^{T} \mathbf{e}\right]}{2 \rho}
$$

A positive $r$ indicates that the decision maker is risk-averse, while a negative $r$ indicates that the decision maker is risk-prone. The risk coefficient equals to zero indicate risk neutrality which means that the decision maker is indifferent of the variability of the outcomes. The magnitude of $r$ is a relative measure of the decision maker attitude towards the variability of the outcomes. For a risk-averse case, the magnitude will measure how far the decision maker will constrain his optimization to avoid uncertain areas of the search space. On the other hand, in a risk-prone case, the magnitude will measure how far the decision maker will relax his optimization to search for a better outcome. The risk coefficient can be kept constant or adjusted adaptively for each iteration during minimizing Eq. A.3. 


\section{APPENDIX B \\ DERIVATION OF THE OBJECTIVE FUNCTION GRADIENT}

The gradient of Eq. 3.2 can be written as,

$$
\nabla_{q} f(\mathbf{q})=\nabla_{q} \mathrm{E}\left[\mathbf{e}^{T} \mathbf{e}\right]+r \nabla_{q} \sigma\left[\mathbf{e}^{T} \mathbf{e}\right]
$$

The first tem represents the gradient of the expect value of the square of $l_{2}$ norm of residuals evaluated for each realization. Because the expected value is a linear operator, the gradient of the expect value operator can be written as,

$$
\nabla_{q} \mathrm{E}\left[\mathbf{e}^{T} \mathbf{e}\right]=2 \mathrm{E}\left[\mathbf{J}^{T} \mathbf{e}\right]
$$

The second term represents the gradient of the standard deviation. By taking the derivative of the standard deviation in Eq. 3, we can reach the following form:

$$
\nabla_{q} \sigma\left[\mathbf{e}^{T} \mathbf{e}\right]=\frac{1}{2} \nabla_{q} \operatorname{Var}\left[\mathbf{e}^{T} \mathbf{e}\right] / \sigma\left[\mathbf{e}^{T} \mathbf{e}\right] .
$$

The variance in Eq. B.3 can be given as:

$$
\operatorname{Var}\left[\mathbf{e}^{T} \mathbf{e}\right]=\mathrm{E}\left[\left(\mathbf{e}^{T} \mathbf{e}\right)^{2}\right]-\mathrm{E}^{2}\left[\mathbf{e}^{T} \mathbf{e}\right]
$$

The gradient of the variance can be written as,

$$
\nabla_{q} \operatorname{Var}\left[\mathbf{e}^{T} \mathbf{e}\right]=4\left\{\mathrm{E}\left[\left(\mathbf{e}^{T} \mathbf{e}\right)\left(\mathbf{J}^{T} \mathbf{e}\right)\right]-\mathrm{E}\left[\mathbf{e}^{T} \mathbf{e}\right] \mathrm{E}\left[\mathbf{J}^{T} \mathbf{e}\right]\right\}
$$

The expression in Eq. B.5 can be written in terms of a covariance as follows:

$$
\operatorname{Cov}\left(\mathbf{e}^{T} \mathbf{e}, \mathbf{J}^{T} \mathbf{e}\right)=\mathrm{E}\left[\left(\mathbf{e}^{T} \mathbf{e}\right)\left(\mathbf{J}^{T} \mathbf{e}\right)\right]-\mathrm{E}\left[\mathbf{e}^{T} \mathbf{e}\right] \mathrm{E}\left[\mathbf{J}^{T} \mathbf{e}\right]
$$


By substituting Eq. B.6 into Eq. B.5, the gradient of the variance can be written as,

$$
\nabla_{q} \operatorname{Var}\left[\mathbf{e}^{T} \mathbf{e}\right]=4 \operatorname{Cov}\left(\mathbf{e}^{T} \mathbf{e}, \mathbf{J}^{T} \mathbf{e}\right)
$$

Now the gradient of the standard deviation can be written as,

$$
\nabla_{q} \sigma\left[\mathbf{e}^{T} \mathbf{e}\right]=2 \frac{\operatorname{Cov}\left(\mathbf{e}^{T} \mathbf{e}, \mathbf{J}^{T} \mathbf{e}\right)}{\sigma\left[\mathbf{e}^{T} \mathbf{e}\right]}
$$

After substituting Eq. B.2 and B.8 into Eq. B.1, we can write the gradient of the objective function as follows,

$$
\nabla_{q} f(\mathbf{q})=2 \mathrm{E}\left[\mathbf{J}^{T} \mathbf{e}\right]+2 r \frac{\operatorname{Cov}\left(\mathbf{e}^{T} \mathbf{e}, \mathbf{J}^{T} \mathbf{e}\right)}{\sigma\left(\mathbf{e}^{T} \mathbf{e}\right)}
$$




\section{APPENDIX C}

\section{DERIVATION OF THE OBJECTIVE FUNCTION HESSIAN}

The Hessian of Eq. 3.2 is given by the following form:

$$
\nabla_{q}^{2} f(\mathbf{q})=\nabla_{q}^{2} \mathrm{E}\left[\mathbf{e}^{T} \mathbf{e}\right]+r \nabla_{q}^{2} \sigma\left[\mathbf{e}^{T} \mathbf{e}\right]
$$

The first term is the Hessian of the expected value of the square of $l_{2}$ norm of the residuals and it can be written as,

$$
\nabla_{q}^{2} \mathrm{E}\left[\mathbf{e}^{T} \mathbf{e}\right]=2 \mathrm{E}\left[\mathbf{J}^{T} \mathbf{J}\right]
$$

The second term is the Hessian of the standard deviation, and it can be written as,

$$
\nabla_{q}^{2} \sigma\left[\mathbf{e}^{T} \mathbf{e}\right]=\frac{1}{2} \frac{\nabla_{q}^{2} \operatorname{Var}\left[\mathbf{e}^{T} \mathbf{e}\right]}{\sigma\left[\mathbf{e}^{T} \mathbf{e}\right]}-\frac{1}{4} \frac{\nabla_{q} \operatorname{Var}\left[\mathbf{e}^{T} \mathbf{e}\right] \nabla_{q}^{T} \operatorname{Var}\left[\mathbf{e}^{T} \mathbf{e}\right]}{\sigma^{3}\left[\mathbf{e}^{T} \mathbf{e}\right]}
$$

Equation C.3 contains the gradient and the Hessian of the variance. In Appendix B, we derived the gradient of the variance. The Hessian of the variance is the gradient of Eq. B.5 and it can be written as,

$$
\nabla_{q}^{2} \operatorname{Var}\left[\mathbf{e}^{T} \mathbf{e}\right]=4\left\{\nabla_{q} \mathrm{E}\left[\left(\mathbf{e}^{T} \mathbf{e}\right)\left(\mathbf{J}^{T} \mathbf{e}\right)\right]-\nabla_{q}\left[\mathrm{E}\left[\mathbf{e}^{T} \mathbf{e}\right] \mathrm{E}\left[\mathbf{J}^{T} \mathbf{e}\right]\right]\right\}
$$

The gradient of the first tem can be written as,

$$
\nabla_{\mathrm{q}} \mathrm{E}\left[\left(\mathbf{e}^{T} \mathbf{e}\right)\left(\mathbf{J}^{T} \mathbf{e}\right)\right]=2 \mathrm{E}\left[\left(\mathbf{J}^{T} \mathbf{e}\right)\left(\mathbf{J}^{T} \mathbf{e}\right)^{T}\right]+\mathrm{E}\left[\left(\mathbf{e}^{T} \mathbf{e}\right)\left(\mathbf{J}^{T} \mathbf{J}\right)\right]
$$

The gradient of the second term can be written as, 


$$
\nabla_{q}\left[\mathrm{E}\left[\mathbf{e}^{T} \mathbf{e}\right] \mathrm{E}\left[\mathbf{J}^{T} \mathbf{e}\right]\right]=2 \mathrm{E}\left[\mathbf{J}^{T} \mathbf{e}\right] \mathrm{E}^{T}\left[\mathbf{J}^{T} \mathbf{e}\right]+\mathrm{E}\left[\left(\mathbf{e}^{T} \mathbf{e}\right)\right] \mathrm{E}\left[\left(\mathbf{J}^{T} \mathbf{J}\right)\right]
$$

By substituting Eqs. C.6 and C.5 into Eq. C.4, the Hessian of the variance can be written as,

$$
\nabla_{q}^{2} \operatorname{Var}\left[\mathbf{e}^{T} \mathbf{e}\right]=4\left\{\begin{array}{l}
2 \mathrm{E}\left[\left(\mathbf{J}^{T} \mathbf{e}\right)\left(\mathbf{J}^{T} \mathbf{e}\right)^{T}\right]-2 \mathrm{E}\left[\mathbf{J}^{T} \mathbf{e}\right] \mathrm{E}^{T}\left[\mathbf{J}^{T} \mathbf{e}\right] \\
+\mathrm{E}\left[\left(\mathbf{e}^{T} \mathbf{e}\right)\left(\mathbf{J}^{T} \mathbf{J}\right)\right]-\mathrm{E}\left[\mathbf{e}^{T} \mathbf{e}\right] \mathrm{E}\left[\mathbf{J}^{T} \mathbf{J}\right]
\end{array}\right\}
$$

The first two terms in Eq. C.7 can be written in terms of the following covariance,

$$
\operatorname{Cov}\left(\mathbf{J}^{T} \mathbf{e}, \mathbf{J}^{T} \mathbf{e}\right)=\mathrm{E}\left[\left(\mathbf{J}^{T} \mathbf{e}\right)\left(\mathbf{J}^{T} \mathbf{e}\right)^{T}\right]-\mathrm{E}\left[\mathbf{J}^{T} \mathbf{e}\right] \mathrm{E}^{T}\left[\mathbf{J}^{T} \mathbf{e}\right]
$$

The last two terms in Eq. C.7 represent the cross-covariance between the square of $l_{2}$ norm of the residuals and the Hessian evaluated for each realization.

$$
\operatorname{Cov}\left(\mathbf{e}^{T} \mathbf{e}, \mathbf{J}^{T} \mathbf{J}\right)=\mathrm{E}\left[\left(\mathbf{e}^{T} \mathbf{e}\right)\left(\mathbf{J}^{T} \mathbf{J}\right)\right]-\mathrm{E}\left[\mathbf{e}^{T} \mathbf{e}\right] \mathrm{E}\left[\mathbf{J}^{T} \mathbf{J}\right]
$$

By substituting Eqs. C.8 and C.9 into Eq. C.7, we reach the following form for the Hessian of the variance.

$$
\nabla_{q}^{2} \operatorname{Var}\left[\mathbf{e}^{T} \mathbf{e}\right]=8 \operatorname{Cov}\left(\mathbf{J}^{T} \mathbf{e}, \mathbf{J}^{T} \mathbf{e}\right)+4 \operatorname{Cov}\left(\mathbf{e}^{T} \mathbf{e}, \mathbf{J}^{T} \mathbf{J}\right)
$$

By substituting Eq. B.7 and C.7 into Eq. C.3, we can reach to the final form of the Hessian of the standard deviation.

$$
\begin{aligned}
\nabla_{q}^{2} \sigma\left[\mathbf{e}^{T} \mathbf{e}\right]= & \frac{4 \operatorname{Cov}\left(\mathbf{J}^{T} \mathbf{e}, \mathbf{J}^{T} \mathbf{e}\right)+2 \operatorname{Cov}\left(\mathbf{e}^{T} \mathbf{e}, \mathbf{J}^{T} \mathbf{J}\right)}{\sigma\left[\mathbf{e}^{T} \mathbf{e}\right]} \\
& -\frac{4 \operatorname{Cov}\left(\mathbf{e}^{T} \mathbf{e}, \mathbf{J}^{T} \mathbf{e}\right) \operatorname{Cov}^{T}\left(\mathbf{e}^{T} \mathbf{e}, \mathbf{J}^{T} \mathbf{e}\right)}{\sigma^{3}\left[\mathbf{e}^{T} \mathbf{e}\right]}
\end{aligned}
$$


After Substituting Eqs. C.2 and C.10 into Eq. C.1 the final form of the Hessian of the objective function is given as follows:

$$
\begin{aligned}
\nabla_{q}^{2} f(\mathbf{q})=2 \mathrm{E}\left[\mathbf{J}^{T} \mathbf{J}\right] & +r \frac{4 \operatorname{Cov}\left(\mathbf{J}^{T} \mathbf{e}, \mathbf{J}^{T} \mathbf{e}\right)+2 \operatorname{Cov}\left(\mathbf{e}^{T} \mathbf{e}, \mathbf{J}^{T} \mathbf{J}\right)}{\sigma\left(\mathbf{e}^{T} \mathbf{e}\right)} \\
& -r \frac{4 \operatorname{Cov}\left(\mathbf{e}^{T} \mathbf{e}, \mathbf{J}^{T} \mathbf{e}\right) \operatorname{Cov}^{T}\left(\mathbf{e}^{T} \mathbf{e}, \mathbf{J}^{T} \mathbf{e}\right)}{\sigma^{3}\left(\mathbf{e}^{T} \mathbf{e}\right)}
\end{aligned}
$$




\section{VITA}

Ahmed Humaid H. Alhuthali completed his Bachelor of Science degree at King Fahd University of Petroleum and Minerals, Department of Electrical Engineering, Saudi Arabia in December 1998, and later obtained his Master of Science degree at the Harold Vance Department of Petroleum Engineering, Texas A\&M University, in August 2003. He earned his Ph.D. at the Harold Vance Department of Petroleum Engineering, Texas A\&M University, in May 2009. His permanent address is Saudi Aramco, Dhahran, 31311, Saudi Arabia. 